JOURNAL OF THE AMERICAN MATHEMATICAL SOCIETY

Volume 20, Number 3, July 2007, Pages 629-673

S 0894-0347(07)00550-4

Article electronically published on January 17, 2007

\title{
PUZZLE GEOMETRY AND RIGIDITY: THE FIBONACCI CYCLE IS HYPERBOLIC
}

\author{
DANIEL SMANIA
}

\section{INTRODUCTION AND RESULTS}

1.1. Complex dynamics results. Rigidity is a striking phenomenon in 1-dimensional dynamics which occurs when a weak equivalence between certain dynamical systems implies a stronger one, for instance, a combinatorial equivalence of the orbit structure implying topological equivalence and topological equivalence implying quasiconformal conjugacy. This phenomenon occurs quite often and has important consequences, as in the proof of the real Fatou conjecture for the quadratic family (Lyubich Lyu97 and Graczyk and Swiatek [GS97]) and the hyperbolicity of the renormalization horseshoe (Lyubich Lyu99).

Usually the hard part is to conclude that two complex dynamical systems are quasiconformally conjugate, once they are topologically conjugate. The first step is to replace the topological conjugacy by another one which is quasiconformal outside the 'complicated' part of the dynamics (the Julia set). This is usually easy. Our first dynamical result is a method to carry out the next step.

Definition 1.1. We say that $f: \bigcup_{i \leq n} U_{i} \rightarrow V$ is a generalized polynomial-like map if

- the sets $U_{i}, i \leq n$, and $V$ are simply connected domains in the complex plane, $U_{i}$ is compactly contained in $V$ and $m(\partial V)=m\left(\partial U_{i}\right)=0$, where $m$ is the 2-dimensional Lebesgue measure;

- $f$ has an analytic extension to a neighborhood of $\bigcup_{i} \bar{U}_{i}$;

- $f: U_{0} \rightarrow V$ is a ramified covering with a unique critical point;

- for each $i \neq 0, f$ is a univalent map from $U_{i}$ onto $V$.

The simply connected components of the domain of $f^{n}$, for each $n>0$, will be called puzzle pieces at level $n$ associated to $f$. If the critical point does not escape $\left(f^{n}(0)\right.$ is well defined, for every $\left.n \geq 0\right)$, there exists a puzzle piece at level $n$ which contains the critical point. This piece is called the critical piece at level $n$ and is denoted by $C_{n}$.

\footnotetext{
Received by the editors April 19, 2005.

2000 Mathematics Subject Classification. Primary 37F30, 37C15, 30C62, 30C65, 37F25, 37F45, 37E20.

Key words and phrases. Fibonacci combinatorics, generalized renormalization, puzzle, rigidity, quasiconformal conjugacy, removability, hyperbolicity.

This work was supported by grants CNPq-200764/01-2, CNPq-472316/2003-6 and FAPESP03/03107-9. Visits to Warwick University and ICTP-Trieste were supported by CNPq-Brazil grant 460110/00-4, Warwick University and ICTP-Trieste.
}

(C)2007 American Mathematical Society Reverts to public domain 28 years from publication 
Theorem 1 (Rigidity Theorem). Let $f_{j}: \bigcup_{i} U_{i}^{j} \rightarrow V^{j}$, with $j=1$, 2, be generalized polynomial-like maps whose critical point 0 does not escape and such that the following hold.

- There exists $\lambda<1$ and an increasing sequence $n_{k}$ so that, if $C_{n}^{j}$ denotes the critical puzzle piece at level $n$ of $f_{j}$, then

- the diameters of the critical puzzle pieces of $f_{1}$ and $f_{2}$ decrease to zero;

- puzzle geometry control: for any $j=1,2, k>0$, there exists $x$ so that

$$
B\left(x, \lambda \cdot \operatorname{diam} C_{n_{k}}^{j}\right) \subset C_{n_{k}}^{j} ;
$$

- complex bounds: for any $j=1,2, k>0$, there exists an annulus $A_{k}^{j} \subset V^{j}$ such that

(1) $\bmod A_{k}^{j} \geq K_{2}$;

(2) the internal boundary of $A_{k}^{j}$ is $\partial C_{n_{k}}^{j}$;

(3) for any $x$ which is not contained in $C_{n_{k}}^{j}$ and $m>0$ minimal so that $f_{j}^{m}(x) \in C_{n_{k}}^{j}$, there exists a neighborhood $V_{j}(x, m)$ of $x$ which is mapped univalently by $f_{j}^{m}$ onto $C_{n_{k}}^{j} \cup A_{k}^{j}$.

- Let $h: \mathbb{C} \rightarrow \mathbb{C}$ be a homeomorphism such that

- $h$ is quasiconformal in $\mathbb{C}-J\left(f_{1}\right)$;

- $h\left(\partial U_{i}^{1}\right)=\partial U_{i}^{2}$ and $h\left(\partial V^{1}\right)=\partial V^{2}$;

- $h$ is a conjugacy between $f_{1}$ and $f_{2}$.

Then $h: \mathbb{C} \rightarrow \mathbb{C}$ is quasiconformal.

Remark 1.1. We can use the weaker assumption that the conjugacy $h$ is defined only in $\mathbb{C}-J\left(f_{1}\right)$, because the "complex bounds" condition on $f_{1}$ and $f_{2}$ implies that $h$ will have a continuous extension to $K\left(f_{1}\right)=J\left(f_{1}\right)$, which is a conjugacy on $J\left(f_{1}\right)$.

Remark 1.2. It is easy to state a similar theorem with many critical points involved.

Remark 1.3. The third property of the annulus $A_{k}^{j}$ follows for instance from the unbranched complex bounds condition

$$
A_{k}^{j} \cap P(f)=\phi, \quad \text { where } P(f):=\overline{\left\{f^{i}(0): i>0\right\}} .
$$

The puzzle geometry control for quadratic-like maps with Fibonacci combinatorics is a consequence from Lyubich Lyu93, and for Fibonacci maps with higher criticalities from Buff $[\mathrm{Bu}]$. Lyubich's result was generalized for an uncountable number of combinatorial types of quadratic polynomials by Perez $[\mathrm{P}$.

Two metric properties are now classic tools to prove rigidity: the "bounded geometry of the postcritical set" and the "linear decay of moduli". The first one was used to prove the rigidity of the infinitely renormalizable real polynomials (with unique critical point) with bounded combinatorics (see Sullivan Sul92 and de Melo and van Strien [dMvS]). The second one was used in the proof of the real Fatou conjecture for quadratic polynomials (Lyubich Lyu97 and Graczyk and Swiatek GS97]). It is quite remarkable that both behaviors can coexist with the puzzle geometry control: the Fibonacci combinatorics is the simplest example. For criticality two, Fibonacci maps have linear decay of modulus (Lyubich and Milnor [LM]); for larger even criticalities, the Fibonacci maps have a postcritical set with bounded geometry (see Bruin, Keller, Nowicki and van Strien [BKNS] for 


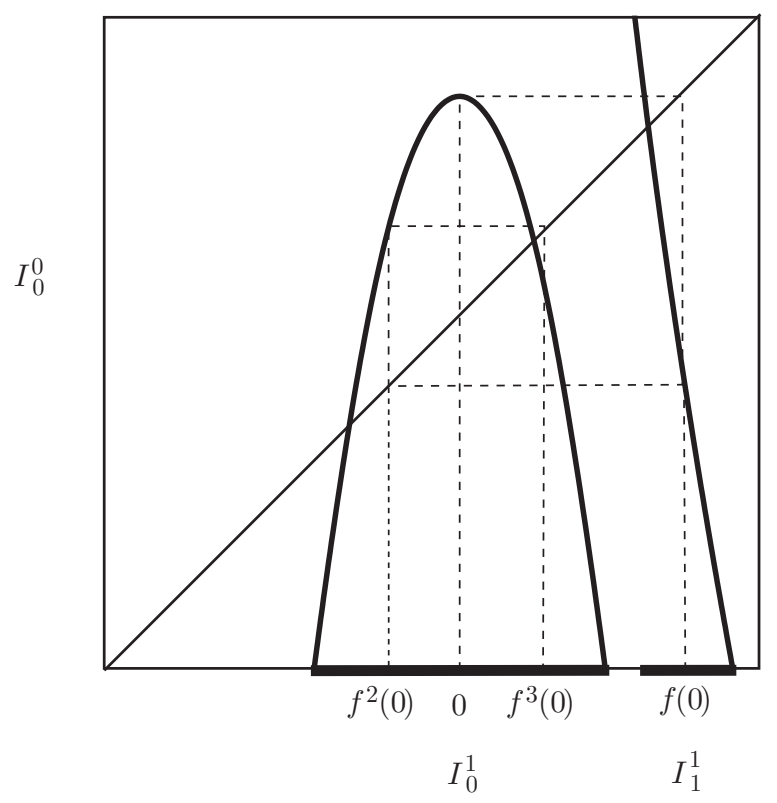

FiguRE 1. A Fibonacci renormalizable map.

high criticalities and Keller and Nowicki [KN]). So the "puzzle geometry control" property seems to be more "primitive" than the two classical properties above.

However, the linear decay of geometry loses its universality for larger criticalities, or even for multimodal maps with quadratic critical points (Swiatek and Vargas [SV]). An even worse fact is the possibility of the existence of real nonrenormalizable combinatorics for higher criticalities and/or many critical points, having neither decay of moduli (at any rate) nor bounded geometry (or even essentially bounded geometry). Our hope is that the puzzle geometry control and complex bounds hold for some of these combinatorial types, whose rigidity is seemingly impossible to prove using previously available methods (indeed, there are recent developments in this direction, as noted in Section 15).

In the second part of this work we will use Theorem 1 to prove that the Fibonacci renormalization operator has a hyperbolic cycle. We introduce the Fibonacci renormalization operator before stating the results.

Maps with Fibonacci combinatorics have already attracted a lot of attention: Lyubich and Milnor [LM]; Lyubich Lyu93; Bruin, Keller, Nowicki and van Strien [BKNS]; Keller and Nowicki [KN]; van Strien and Nowicki vsN94]; Buff [Bu]. The main points are that

- the Fibonacci combinatorics is the simplest one which admits wild attractors for high criticalities;

- for critical order larger than two, the Fibonacci combinatorics have bounded geometry (no decay of geometry);

- the Fibonacci combinatorics is the simplest combinatorics which is infinitely renormalizable in the generalized sense. 
Let $d$ be an even natural number larger than two ( $d$ will be fixed for the rest of this work). If $\alpha$ is a complex number and $A$ a subset of the complex plane, denote $\alpha A:=\{\alpha \cdot x: x \in A\}$.

Let $f$ be a real analytic map defined in two intervals, a symmetric interval $I_{0}^{1}$ and $I_{1}^{1}$. Denote $I_{0}^{0}:=f\left(I_{1}^{1}\right)$. Assume the following.

- $f: I_{0}^{1} \rightarrow I_{0}^{0}$ has zero as its unique critical point, which is a maximum. Moreover, the degree of zero is $d$ and $f\left(\partial I_{0}^{1}\right) \subset \partial I_{0}^{0}$.

- $f: I_{1}^{1} \rightarrow I_{0}^{0}$ does not have critical points and $f\left(\partial I_{1}^{1}\right) \subset \partial I_{0}^{0}$.

We will say that $f$ is Fibonacci renormalizable if

- $f(0) \in I_{1}^{1}$

- $f^{2}(0) \in I_{0}^{1}$

- $f^{3}(0) \in I_{0}^{1}$

- the connected component of the domain of $f^{2}$ which contains the critical point also contains a fixed point, denoted $\beta_{f}$, so that $D f^{2}\left(\beta_{f}\right)<0$.

Denote by $I_{0}^{2}$ the connected component of the domain of the first return map to $I_{0}^{1}$ which contains 0 and by $I_{1}^{2}$ the connected component of the domain of the first return map to $I_{0}^{1}$ which contains $f^{2}(0)$. Note that $I_{0}^{2} \cap I_{1}^{2}=\phi$. The Fibonacci renormalization of $f$, denoted $\mathcal{R} f$, is defined as the map

$$
\mathcal{R} f: \frac{1}{\beta_{f}} I_{0}^{2} \cup \frac{1}{\beta_{f}} I_{1}^{2} \rightarrow \frac{1}{\beta_{f}} I_{0}^{1},
$$

where $\mathcal{R} f(x)$ is equal to

$$
\frac{1}{\beta_{f}} f^{2}\left(\beta_{f} \cdot x\right)
$$

on $1 / \beta_{f} I_{0}^{2}$ and

$$
\frac{1}{\beta_{f}} f\left(\beta_{f} \cdot x\right)
$$

on $1 / \beta_{f} I_{1}^{2}$. If $\mathcal{R}^{n} f$ is defined for every $n \geq 0$, we say that $f$ is infinitely renormalizable in the Fibonacci sense.

In van Strien and Nowicki vSN94, it was proved that this operator has a unique periodic orbit of period two, the Fibonacci cycle. Indeed, if $\tilde{f}_{1}: I \cup J \rightarrow T$ is one of the maps in the periodic orbit, then the map $\tilde{f}_{2}$ defined as $\tilde{f}_{1}$ on $I$ and $-f_{1}$ on $J$ is exactly $\mathcal{R} \tilde{f}_{1}$ (see Buff $[\mathrm{Bu}]$ ). Making a slight modification of the Fibonacci renormalization operator, we can consider these maps as fixed points of the modified operator (see Section 14). The following remark is very important: Since these maps are almost the same, a distinction between the two maps is not important, so sometimes we will denote both of them by $\tilde{f}$. For instance $|g-\tilde{f}|$ denotes $\min \left\{\left|g-\tilde{f}_{1}\right|,\left|g-\tilde{f}_{2}\right|\right\}$.

The map $\tilde{f}$ has a generalized polynomial-like extension $\tilde{f}: \bar{U}_{0} \cup \bar{U}_{1} \rightarrow \bar{V}$, where $0 \in U_{0}$. Let $\mathcal{B}_{\text {nor }}\left(U_{0} \cup U_{1}\right)$ be the affine space of complex analytic functions on $U_{0} \cup U_{1}$ with continuous extension to $\overline{U_{0} \cup U_{1}}$ and an appropriate normalization, endowed with the sup norm. Selecting $U_{0}$ and $U_{1}$ in an appropriate way, we will define an operator $\mathcal{R}: B_{\epsilon_{0}}(\tilde{f}) \rightarrow \mathcal{B}_{\text {nor }}\left(U_{0} \cup U_{1}\right)$, where $B_{\epsilon_{0}}(\tilde{f}) \subset \mathcal{B}_{\text {nor }}\left(U_{0} \cup U_{1}\right)$ is the $\epsilon_{0}$-ball around $\tilde{f}$, which coincides with the Fibonacci renormalization operator above defined in the real trace of the real functions in $B_{\epsilon_{0}}(\tilde{f})$, so that we have the following. 
Theorem 2 (Hyperbolicity). The Fibonacci renormalization operator $\mathcal{R}$, defined in a small neighborhood of $\tilde{f}$ in $\mathcal{B}_{\text {nor }}\left(U_{1} \cup U_{2}\right)$, is a compact operator with a hyperbolic cycle $\left\{\tilde{f}_{1}, \tilde{f}_{2}\right\}$ with codimension one stable manifold $W^{s}(\tilde{f})$. Furthermore, if $f$ is a real analytic map that is infinitely renormalizable in the Fibonacci sense, then there exists $N=N(f)$ so that $\mathcal{R}^{N} f$ has a complex analytic extension in $W^{s}(\tilde{f})$.

Let $\mathcal{D}$ be the set of complex analytic families $f: \overline{\mathbb{D}} \times \bar{U} \rightarrow \mathbb{C}$, with $f_{\lambda} \in \mathcal{B}_{\text {nor }}\left(U_{1} \cup\right.$ $U_{2}$ ), so that for each $\lambda \in[-1,1]$ the map $f_{\lambda}$ is even and preserves the real line. The hyperbolicity of the Fibonacci renormalization operator implies, after a short argument in Section 14, the following universality result:

Theorem 3 (Universality). Let d be an even natural number larger than two. Then there exists $\gamma=\gamma_{d}>1$ so that for an open and dense set of families $f_{\lambda} \in \mathcal{D}$ the following holds: if $f_{\lambda_{\infty}}$, with $\lambda_{\infty} \in\left[-\frac{1}{2}, \frac{1}{2}\right]$, has a polynomial-like restriction which is quasiconformally conjugate with either $\tilde{f}_{1}$ or $\tilde{f}_{2}$, then there exist $\delta>0, N \in \mathbb{N}$, and a sequence $\lambda_{n} \in \mathbb{R}$ so that

- the sequence $\lambda_{n}$ converges to $\lambda_{\infty}$;

- for $n \geq N, \lambda_{n}$ is the unique parameter such that $\left|\lambda_{n}-\lambda_{\infty}\right| \leq \delta$ and whose map $f_{\lambda_{n}}$ has a superattractor with period $S_{n}$ (the Fibonacci sequence) and whose closest returns of the critical point are exactly $S_{i}, i<n$;

- we have

$$
\frac{\lambda_{n}-\lambda_{n+1}}{\lambda_{n+1}-\lambda_{n+2}} \rightarrow \gamma
$$

A similar statement holds for the family $x^{d}+c$ (perturbations are not necessary in this case).

1.2. Removability result. Removability-type results try to find useful properties for a homeomorphism on $\mathbb{C}$ that is quasiconformal outside a set $K$ that imply quasiconformality everywhere. We will state a new abstract removability-type result: this result is a major step in the proof of Theorem 1 Before stating it, we need to introduce some notation. We will consider only measurable functions.

In this section, we will reserve the word family to denote families of domains: a domain is an open and connected set in $\mathbb{C}$. Moreover, we will assume that all these domains have boundaries with zero 2-dimensional Lebesgue measure. Sometimes we will deal with families of centered domains: this is a family of pairs $(A, x)$, where $x \in A$. We will say that $x$ is a center of $A$. It may occur in a given family that $A$ has many centers and that a point $x$ is the center of many domains. Recall that $m(A)$ denotes the 2-dimensional Lebesgue measure of $A$.

Definition 1.2. A family $\mathcal{F}$ covers a set $X \subset \mathbb{C}$ in arbitrary small scales if for any $x \in X$ and $\epsilon>0$ there exists $A \in \mathcal{F}$ such that $x \in A$, diam $A \leq \epsilon$ and $\partial A \cap X=\phi$ for $A \in \mathcal{F}$.

Definition 1.3. A centered family $\mathcal{F}$ covers a set $X \subset \mathbb{C}$ in all (small) scales if for any $x \in X$ there exists $C=C(x)>1$ and $\epsilon_{0}=\epsilon_{0}(x)$ so that for any $\epsilon \leq \epsilon_{0}$ the point $x$ is the center of one domain $A \in \mathcal{F}$ satisfying

$$
\frac{1}{C} \epsilon \leq \operatorname{diam} A \leq C \epsilon
$$

Moreover $X \cap \partial A=\phi$ for $A \in \mathcal{F}$. If $C$ and $\epsilon_{0}$ do not depend on $x$, we say that $\mathcal{F}$ covers a set $X$ in all scales in a $\left(C, \epsilon_{0}\right)$-uniform way. 
Definition 1.4. We say that a family $\mathcal{F}$ has uniform bounded geometry if there exists $\lambda<1$ so that for every $A \in \mathcal{F}$ there exists $x \in A$ such that

$$
B(x, \lambda \cdot \operatorname{diam} A) \subset A .
$$

Definition 1.5. We say that a centered family $\mathcal{F}$ has pointwise bounded geometry if for any $x \in \mathbb{C}$ there exists $C=C(x)<1$ so that if $(A, x) \in \mathcal{F}$, then $B(x, C \cdot \operatorname{diam} A) \subset A$. The centered family $\mathcal{F}$ has $C$-uniform bounded geometry if $C$ does not depend on $x$.

Definition 1.6. We say that a family of domains $\mathcal{F}$ is a Markov family if we have the following.

- For $A_{1}$ and $A_{2}$ in $\mathcal{F}$, one of the following statements holds:

$-A_{1} \cap A_{2}=\phi$

$-A_{1} \subset A_{2}$

$-A_{2} \subset A_{1}$.

- Each subfamily $\mathcal{F}^{\prime} \subset \mathcal{F}$ is finite if it satisfies the following properties:

- The inclusion is a total order in $\mathcal{F}^{\prime}$ : if $A_{1}, A_{2} \in \mathcal{F}^{\prime}$, then either $A_{1} \subset A_{2}$ or $A_{2} \subset A_{1}$.

- There exists $A_{0} \in \mathcal{F}$ such that $A_{0} \subset A$ for any $A \in \mathcal{F}^{\prime}$.

Markov families arise in a very natural way in complex dynamics: the family of the puzzle pieces of any level is a Markov family. Note that any subfamily of a Markov family is a Markov family itself. The main property of Markov families is the following: if a Markov family $\mathcal{F}$ covers a set $X$, then there exists a subfamily $\mathcal{F}^{\prime} \subset \mathcal{F}$ which covers each point of $X$ once and each point of $\mathbb{C}$ at most once.

Let $h$ be a homeomorphism in the complex plane and let $\mathcal{F}$ be a (centered) family. Define

$$
h(\mathcal{F})=\{h(A): A \in \mathcal{F}\},
$$

if $\mathcal{F}$ is a family, or define

$$
h(\mathcal{F})=\{(h(A), h(x)):(A, x) \in \mathcal{F}\},
$$

if $\mathcal{F}$ is a centered family.

Theorem 4 (Removability Theorem). Let $h: \mathbb{C} \rightarrow \mathbb{C}$ be a homeomorphism and let $X \subset \mathbb{C}$ be a compact set. Suppose that $h$ is quasiconformal in $\mathbb{C}-X$. Assume that there exist a decomposition $X=X_{1} \cup X_{2}$, a family $\mathcal{F}_{1}$ and a centered family $\mathcal{F}_{2}$ such that

- $\mathcal{F}_{1}$ is a Markov family,

- $\mathcal{F}_{1}$ covers $X_{1}$ in arbitrary small scales,

- $\mathcal{F}_{1}$ and $h\left(\mathcal{F}_{1}\right)$ have uniform bounded geometry,

and

- $X_{2}$ has zero 2-dimensional Lebesgue measure,

- $\mathcal{F}_{2}$ covers $X_{2}$ in all scales,

- $\mathcal{F}_{2}$ and $h\left(\mathcal{F}_{2}\right)$ have pointwise bounded geometry.

Then $h$ is quasiconformal in $\mathbb{C}$.

Remark 1.4. The same conclusion holds if we add another exceptional set $X_{3}$ with $\sigma$-finite 1-dimensional Hausdorff measure. 
Remark 1.5. The same proof works replacing $\mathbb{C}$ by $\mathbb{R}^{n}, n \geq 2$, and quasiconformal by quasisymmetric, assuming that $X_{2}$ has zero $n$-dimensional Lebesgue measure and $X_{3}$ has $\sigma$-finite $(n-1)$-dimensional Hausdorff measure.

Theorem 4 is an almost straightforward generalization of the main result in Kallunki and Koskela [KK00]. In their proof they use the so-called Besicovitch Theorem, which is replaced here by the Markov property. For completeness we present an almost self-contained proof in Section 3. Apart from the above definitions about families, Section 3 is independent of the rest of this work.

In the application of Theorem4 to prove Theorem[1 (see Section 41), $X$ will be the filled-in Julia set (with empty interior) of a generalized polynomial-like map, $X_{1}$ will be the points in the Julia set whose forward orbit accumulates on the critical point and $X_{2}$ will be the set of points whose forward orbit is far from the critical point. The family $\mathcal{F}_{1}$ will be defined as the family of univalent pullbacks of critical puzzle pieces with bounded geometry. The complex bounds and the Koebe distortion lemma will provide the uniform bounded geometry. For each point $x \in X_{2}$, there exists a finite number of puzzle pieces (depending on $x$ ) so that each domain in $\mathcal{F}_{2}$ which covers $x$ is a univalent pullback (with bounded distortion) of one of these domains. This will give us the pointwise bounded geometry.

\section{Outline OF THE WORK}

Part 1. In the first part of this work (Sections 3-4) we prove Theorem 4 and Theorem 1. We prove Theorem 4 in Section 3 and then deduce Theorem 1 in Section 4 .

The naive argument of the proof of Theorem 1 is the following: since we know that the shapes of certain combinatorially defined critical puzzle pieces of both maps $f_{1}$ and $f_{2}$ are under control, we have, by assumption, that the conjugacy $h$ does not distort certain critical puzzle pieces. Using the dynamics, we transport this control of puzzle distortion to any point whose forward orbit accumulates on the critical point (here some "complex bounds" are needed). We can deal with the points whose orbits do not accumulate on the critical point in another way (see below). So we conclude that the conjugacy does not distort a lot of puzzle pieces, around a lot of points in the Julia set (this is done in Section 4): we can expect that this conjugacy is very regular.

To make this argument rigorous, we need Theorem 4. Indeed, the recent proof by Przytycki and Rohde [PR99] that the Julia sets of CE holomorphic repellers are dynamically removable draws our attention to a result by Heinonen and Koskela HK95, which claims that, to prove that a homeomorphism is quasiconformal, it is sufficient to find, for each point in the domain, a sequence of circles centered on that point, whose diameter goes to zero, so that the geometry of the image of these circles by the homeomorphism is under control. Since we will not use circles, but puzzle pieces (which are hardly circles), a new kind of "removability" result is necessary: certain coverings of sets by discs have very special properties, as in the Besicovitch Theorem. But the advantage of the puzzle pieces is that they satisfy a "Markov property" (see Definition 1.6), which substitutes the Besicovich Theorem, and moreover they are dynamically defined, which is very unusual for circles!

Unfortunately, we cannot transport the information about the geometry of the critical puzzle pieces in the principal nest and their images to all points in the Julia set, since there are points whose orbits do not accumulate the critical point. The 
solution to this problem is that the set of these problematic points is small (zero Lebesgue measure: see Corollary 4.2), so we can be (in some sense) less exigent with them: we need only a pointwise control of distortion, which is obtained using the expansion of the dynamics far from the critical point (Corollary 4.1). Here an argument developed by Kallunki and Koskela [KK00 will be a useful tool. So a new removability-type theorem arises (Theorem 4).

Part 2. The goal of the second part (Sections 5-9) is to show that the local stable manifold of the Fibonacci cycle

$$
W_{\delta}^{s}(\tilde{f}):=\left\{f:\left|\mathcal{R}^{i} f-\tilde{f}\right|<\delta, \text { for all } i \in \mathbb{N}\right\}
$$

is contained in the hybrid class of $\tilde{f}$ (see Theorem 5 ). Indeed in Section 12 (Part 3 ) we will improve this result, proving that in the hybrid class of $\tilde{f}$ the Fibonacci renormalization operator is contracting, so the renormalizations of maps in the hybrid class converges to the Fibonacci cycle exponentially fast. These two results give a characterization of the stable manifold: this result will be essential in the proof of the hyperbolicity of the Fibonacci cycle in the third part.

In Section [6] we study the maps in the Fibonacci cycle, using results from Buff $\mathrm{Bu}$. We are especially interested in the shape of the puzzle pieces in the principal nest of these maps: after a proper normalization of the maps and domains in the principal nest of Fibonacci maps, we obtain a (fixed) hyperbolic polynomial-like map. As a consequence, the shapes of the puzzle pieces in the principal nest are very close to the shape of the Julia set of such hyperbolic map $\mathrm{Bu}$.

A complex analytic version of the Fibonacci renormalization operator is defined in Section 7 In Section 8, we prove that the geometry of critical puzzle pieces in the principal nest of maps whose renormalizations are very close to the Fibonacci cycle is under control. Indeed their shapes are close to the shape of the Julia set of the hyperbolic polynomial-like map cited above (Proposition 8.1). Roughly speaking, this follows from the stability of the Julia sets of hyperbolic maps.

In Section 9 we prove that, if the orbit of a map $f$ under the Fibonacci renormalization operator is always very close to the Fibonacci cycle $\tilde{f}$, then there exists a hybrid conjugacy between that map and one of the maps in the cycle. The first step is to construct a topological conjugacy $h$ that is quasiconformal outside their Julia sets (Proposition 9.1).

The next step is to prove that the conjugacy is indeed quasiconformal in the Julia set. Unfortunately, all the previous methods to prove this kind of result for non-renormalizable (in the classical sense) and non-necessarily real maps are based on the "modulus divergence property", "linear decay of moduli property" or that the dynamics has some "expansivity" (Collet-Eckmann, summability or RiveraLetelier's decay of geometry). None of these properties hold for Fibonacci maps with criticalities larger than two. So we need a new ingredient, i.e., the control of the shape of the puzzle pieces, and Theorem 1 implies that the conjugacy is quasiconformal everywhere.

Part 3. In the third part (Sections 10-15) we prove the hyperbolicity of the Fibonacci cycle and the universality result.

In Section 10 we give an introduction to the theory of infinitesimal perturbations of maps developed in Lyubich Lyu99 and Avila, Lyubich and de Melo ALdM], with minor modifications, and in Section 11 we introduce some basic facts about 
induced transformations: the Fibonacci renormalization operator is an example of an induced transformation. In Section 12, the exponential contraction of the Fibonacci renormalization operator in the hybrid class of the maps in the Fibonacci cycle is proved (see Corollary 12.2), using the non-existence of invariant line fields in the Fibonacci tower vSN94 and a simple argument introduced in Sm02. Finally, in Section 13 we prove that the Fibonacci cycle is hyperbolic (Theorem 21). The proof of the hyperbolicity of the Fibonacci cycle goes like this: Using the Small Orbits Theorem Lyu99 and the exponential contraction on the hybrid classes, to prove that the Fibonacci cycle is hyperbolic, it is sufficient to prove that maps whose Fibonacci renormalizations are all very close to the Fibonacci cycle are indeed quasiconformally conjugate to one of the maps in the Fibonacci cycle. So the hyperbolicity is reduced to the rigidity problem solved in the second part.

In Section 14 we derive the universality result (Theorem 3). Future developments and open questions are described in Section 15 ,

\section{Part 1. Puzzle Geometry and Rigidity}

\section{Removability Theorem}

We are going to prove Theorem 4 . The following proof is almost self-contained. We follow closely the ideas of S. Kallunki and P. Koskela KK00 and J. Heinonen and P. Koskela [HK95]. Denote $B\left(z_{0}, r\right)=\left\{z:\left|z-z_{0}\right| \leq r\right\}, S\left(z_{0}, r\right)=$ $\left\{z:\left|z-z_{0}\right|=r\right\}$ and define $\pi_{z_{0}}: \mathbb{C}-\{0\} \rightarrow S\left(z_{0}, 1\right)$ by $\pi_{z_{0}}(z)=\frac{z-z_{0}}{\mid z-z_{0}}+z_{0}$. Denote by $1_{A}$ the characteristic function of the set $A$. Here $m$ is the 2-dimensional Hausdorff measure and $m_{1}$ is the 1-dimensional Hausdorff measure.

Proof of Theorem 4. Let $x_{0}$ be an arbitrary point and $r_{0}>0$. Define

$$
\begin{aligned}
l_{h}\left(x_{0}, r_{0}\right) & :=\inf \left\{\left|h(x)-h\left(x_{0}\right)\right|:\left|x-x_{0}\right| \geq r_{0}\right\}, \\
L_{h}\left(x_{0}, r_{0}\right) & :=\sup \left\{\left|h(x)-h\left(x_{0}\right)\right|:\left|x-x_{0}\right| \leq r_{0}\right\} .
\end{aligned}
$$

We need to prove that

$$
\frac{L_{h}\left(x_{0}, r_{0}\right)}{l_{h}\left(x_{0}, r_{0}\right)} \leq H
$$

for some universal $H$. Denote $L=L_{h}\left(x_{0}, r_{0}\right)$ and $l=l_{h}\left(x_{0}, r_{0}\right)$. Define $A:=$ $h^{-1}\left(\left\{y: l \leq\left|y-h\left(x_{0}\right)\right| \leq L\right\}\right)-X_{2}$. Since $h$ is quasiconformal outside $X$, there exists $\lambda<1$ such that, for every $x$ outside $X$, we can find $r_{0}(x)>0$ so that

$$
B(h(x), \lambda \operatorname{diam} h(B(x, r))) \subset h(B(x, r)),
$$

for $r<r_{0}(x)$. Let $j_{0}$ be minimal such that $L 2^{-j_{0}}<l$. We will define a family of closed discs $\mathcal{B}_{j}$ and a Markov family $\mathcal{M}_{j}$ in the following way:

- A disc $B(x, R)$ belongs to $\mathcal{B}_{j}$, with $j \leq j_{0}+1$, if

$-x \in A-X$,

$-r<r_{0}(x)$ and

$-h(B(x, r))$ is contained in $R_{j}:=\left\{y: L 2^{-(j+1)}<\left|y-h\left(x_{0}\right)\right|<L\left(2^{-j}+\right.\right.$ $\left.\left.2^{-\left(j_{0}+1\right)}\right)\right\}$ and $\operatorname{diam} h(B(x, r)) \leq L 2^{-100 j_{0}}$.

- A piece $M \in \mathcal{F}_{1}$ belongs to $\mathcal{M}_{j}$, with $j \leq j_{0}+1$, if $h(M)$ is contained in $R_{j}$ and $\operatorname{diam} h(M) \leq L 2^{-100 j_{0}}$. 
Note that $\bigcup_{j} \mathcal{B}_{j}$ covers $A-X$ and $\bigcup_{j} \mathcal{M}_{j}$ covers $A \cap X_{1}$. Applying the Besicovitch Covering Theorem to $\bigcup_{j} \mathcal{B}_{j}$, we can

- replace $\mathcal{B}_{j}$ by an enumerable subfamily, denoted by the same symbol $\mathcal{B}_{j}$, such that

$$
1_{A-X} \leq \sum_{j \leq j_{0}} \sum_{B \in \mathcal{B}_{j}} 1_{B} \leq C
$$

- moreover, each family $\mathcal{B}_{j}$ can be decomposed into $Q$ subfamilies $\mathcal{B}_{j}^{i}, i=$ $1, \ldots, Q$, where $Q$ does not depend on anything, so that

if $B_{1} \in \mathcal{B}_{j_{1}}^{i}$ and $B_{2} \in \mathcal{B}_{j_{2}}^{i}$, then either $B_{1} \cap B_{2}=\phi$ or $j_{1}=j_{2}$ and $B_{1}=B_{2}$.

Similarly, since $\mathcal{F}_{1}$ is a Markov family, we can replace $\mathcal{M}_{j}$ by a subfamily, if necessary, so that

$$
\sum_{j \leq j_{0}} \sum_{M \in \mathcal{M}_{j}} 1_{M} \leq 1
$$

almost everywhere in $\mathbb{C}$ and

$$
\sum_{j \leq j_{0}} \sum_{M \in \mathcal{M}_{j}} 1_{M}=1
$$

in $A \cap X_{1}$. Note that there exists a universal constant $C>1$ such that, for any $M \in \bigcup_{j} \mathcal{M}_{j}$, there exists a $\operatorname{disc} B^{M}$ which satisfies

- $\operatorname{diam} B^{M} \sim \operatorname{diam} M$,

- $\operatorname{diam} C B^{M}>2 \operatorname{diam} M$,

- $B^{M} \subset M \subset C B^{M}$.

Define

$$
\begin{gathered}
\rho:=\left(\log \frac{L}{l}\right)^{-1} \sum_{j \leq j_{0}} \sum_{B \in \mathcal{B}_{j}} \frac{\operatorname{diam} h(B)}{\operatorname{dist}\left(h(B), h\left(x_{0}\right)\right)} \frac{1}{\operatorname{diam~} B} 1_{2 B} \\
+\left(\log \frac{L}{l}\right)^{-1} \sum_{j \leq j_{0}} \sum_{M \in \mathcal{M}_{j}} \frac{\operatorname{diam} h(M)}{\operatorname{dist}\left(h(M), h\left(x_{0}\right)\right)} \frac{1}{\operatorname{diam~M}} 1_{C B^{M} .}
\end{gathered}
$$

We claim that

$$
\int \rho^{2} d m \leq\left(\log \frac{L}{l}\right)^{-1}
$$

Denote

$$
\begin{aligned}
a_{B} & :=\frac{\operatorname{diam} h(B)}{\operatorname{dist}\left(h(B), h\left(x_{0}\right)\right)} \frac{1}{\operatorname{diam} B}, \\
a_{M} & :=\frac{\operatorname{diam} h(M)}{\operatorname{dist}\left(h(M), h\left(x_{0}\right)\right)} \frac{1}{\operatorname{diam} M} .
\end{aligned}
$$

By Lemma 4.2 in B],

$$
\begin{gathered}
\int \rho^{2} d m=\left(\log \frac{L}{l}\right)^{-2}\left(\int\left(\sum_{j \leq j_{0}} \sum_{B \in \mathcal{B}_{j}} a_{B} 1_{2 B}+\sum_{j \leq j_{0}} \sum_{M \in \mathcal{M}_{j}} a_{M} 1_{C B^{M}}\right)^{2} d m\right) \\
\leq C\left(\log \frac{L}{l}\right)^{-2}\left(\int\left(\sum_{j \leq j_{0}} \sum_{B \in \mathcal{B}_{j}} a_{B} 1_{B}\right)^{2} d m+\int\left(\sum_{j \leq j_{0}} \sum_{M \in \mathcal{M}_{j}} a_{M} 1_{B^{M}}\right)^{2} d m\right) \\
\leq C\left(\log \frac{L}{l}\right)^{-2}\left(\sum_{j \leq j_{0}} \sum_{B \in \mathcal{B}_{j}} a_{B}^{2} m(B)+\sum_{j \leq j_{0}} \sum_{M \in \mathcal{M}_{j}} a_{M}^{2} m(M)\right) .
\end{gathered}
$$


By assumption

$$
\frac{m(h(B))}{(\operatorname{diam} h(B))^{2}}, \frac{m(h(M))}{(\operatorname{diam} h(M))^{2}}, \text { and } \frac{m(M)}{(\operatorname{diam} M)^{2}}
$$

are at a definite distance from zero and infinity. We obtain

$$
\begin{aligned}
\int \rho^{2} d m & \leq C\left(\log \frac{L}{l}\right)^{-2}\left(\sum_{j \leq j_{0}} \sum_{B \in \mathcal{B}_{j}} \frac{m(h(B))}{\operatorname{dist}\left(h(B), h\left(x_{0}\right)\right)^{2}}+\sum_{j \leq j_{0}} \sum_{M \in \mathcal{M}_{j}} \frac{m(h(M))}{\operatorname{dist}\left(h(M), h\left(x_{0}\right)\right)^{2}}\right) \\
& \leq C\left(\log \frac{L}{l}\right)^{-2}\left(\sum_{j \leq j_{0}}\left(2^{-j} L\right)^{-2} \sum_{B \in \mathcal{B}_{j}} m(h(B))+\sum_{j \leq j_{0}}\left(2^{-j} L\right)^{-2} \sum_{M \in \mathcal{M}_{j}} m(h(M))\right) \\
& \leq C\left(\log \frac{L}{l}\right)^{-1} .
\end{aligned}
$$

The last inequality is a consequence of the following:

- $h\left(\mathcal{B}_{j}\right)$ covers each point in $R_{j}$ at most a universal number of times.

- $h\left(\mathcal{M}_{j}\right)$ covers almost each point in $R_{j}$ at most once.

- The measure of $R_{j}$ is commensurable with $\left(2^{-j} L\right)^{2}$.

- Assume that $j_{0}>10$; otherwise there is nothing to prove. Then $j_{0}$ is smaller than $C \log \frac{L}{l}$.

Without loss of generality (see [KK00]), we can assume that there are subcontinua $F_{1} \subset h^{-1}\left\{y:\left|y-h\left(x_{0}\right)\right|=l\right\}$ and $F_{2} \subset h^{-1}\left\{y:\left|y-h\left(x_{0}\right)\right|=L\right\}$ such that $\operatorname{dist}\left(F_{1}, F_{2}\right)=2 \operatorname{diam} F_{1}=2 \operatorname{diam} F_{2}=\frac{1}{2}$. Select $x_{i} \in F_{i}$ so that $\left|x_{1}-x_{2}\right|=\operatorname{dist}\left(F_{1}, F_{2}\right)$ and denote by $S$ the unique segment of length one whose mid-point is the intersection point of $S$ and the segment between $x_{1}$ and $x_{2}$ and, moreover, $S$ cuts this segment perpendicularly, exactly through the middle. Using Lemma 2.1 in KK00, to get a lower bound for $\int \rho^{2} d m$, it is sufficient to prove that, for fixed $x \in F_{1}$ and $y \in F_{2}$,

$$
\int_{\gamma(x, y, z)} \rho d m \geq \delta>0
$$

for almost every $z \in S$. Here $\gamma(x, y, z)$ is the union of the segments between $x$ and $z$ and $y$ and $z$. Indeed,

$$
\begin{gathered}
\int_{\gamma(x, y, z)} \rho d m \geq C\left(\log \frac{L}{l}\right)^{-1} \sum_{j \leq j_{0}}\left(2^{-j} L\right)^{-1} \sum_{B \in \mathcal{B}_{j},} \sum_{B \cap \gamma(x, y, z) \neq \phi} \operatorname{diam} h(B) \\
+C\left(\log \frac{L}{l}\right)^{-1} \sum_{j \leq j_{0}}\left(2^{-j} L\right)^{-1} \sum_{M \in \mathcal{M}_{j},} \operatorname{diam} h(M) .
\end{gathered}
$$

Assume for a moment that $m_{1}\left(h(\gamma(x, y, z)) \cap h\left(X_{2}\right)\right)=0$. Then

$$
\sum_{B \in \mathcal{B}_{j}, B \cap \gamma(x, y, z) \neq \phi} \operatorname{diam} h(B)+\sum_{M \in \mathcal{M}_{j},} \sum_{M \cap \gamma(x, y, z) \neq \phi} \operatorname{diam} h(M) \geq C 2^{-j} L .
$$

Since $j_{0} \sim \log \frac{L}{l}$, we have obtained the lower estimate for $\int_{\gamma(x, y, z)} \rho d m$.

So we need to prove that for $x$ and $y$ fixed, $m_{1}\left(h(\gamma(x, y, z)) \cap h\left(X_{2}\right)\right)=0$ for almost every $z \in S$. Indeed, fix $z_{0} \in \mathbb{C}$ and denote $E_{\sigma}=\left\{z_{0}+\lambda \sigma: 1 \leq \lambda \leq 2\right\} \cap X_{2}$. We claim that $m_{1}\left(h\left(E_{\sigma}\right)\right)=0$, for almost every $\sigma \in \mathbb{S}^{1}$. Since $m\left(X_{2}\right)=0$, we have that, for almost every $\sigma \in \mathbb{S}^{1}, m_{1}\left(E_{\sigma}\right)=0$. We can decompose $E_{\sigma}$ into a countable number of parts (denote each part by $E_{\sigma}$, again), so that for each part there exist constants $C_{1}>1, C_{2}, C_{3}<1$ and $r_{0}>0$ so that for any $x \in E_{\sigma}$ and $r<r_{0}$, there 
exists $(A, x) \in \mathcal{F}_{2}$ satisfying

- $\frac{1}{C_{1}} r \leq \operatorname{diam} A \leq C_{1} r$,

- $B\left(x, C_{2} \operatorname{diam} A\right) \subset A$,

- $B\left(h(x), C_{3} \operatorname{diam} h(A)\right) \subset h(A)$.

By a simple argument as in the proof of Lemma 2.4 in KK00, it is sufficient to prove the following: there exists $C=C\left(\sigma, C_{1}, C_{2}, C_{3}\right)>0$ such that if $F$ is a compact set in $z_{0}+\sigma \mathbb{R}$, then $m_{1}\left(h\left(F \cap E_{\sigma}\right)\right) \leq C\left(m_{1}(F)\right)^{1 / 2}$. To prove this, find intervals of disjoint interior $I_{i} \subset z_{0}+\sigma \mathbb{R}, i=1, \ldots, p$, with length $r \leq C_{2} / C_{1} r_{0}$ so that $F \subset \bigcup_{i} I_{i}$ and $p r \leq 2 m_{1}(F)$. For each $i$ so that $E_{\sigma} \cap I_{i} \neq \phi$, select $x_{i} \in I_{i} \cap E$. Then there exists $\left(A_{i}, x_{i}\right) \in \mathcal{F}_{2}$ satisfying

- $I_{i} \subset A_{i}$,

- $\frac{1}{C} r \leq \operatorname{diam} A_{i} \leq C r$.

So $h(F) \subset \bigcup_{i} h\left(A_{i}\right)$. Note that the family $\left\{h\left(A_{i}\right)\right\}_{i}$ covers each point in $\mathbb{C}$ at most $N\left(C_{1}, C_{2}\right)$ times. Then

$$
\begin{gathered}
\left(\sum_{i} \operatorname{diam} h\left(A_{i}\right)\right)^{2} \leq C p \sum_{i}\left(\operatorname{diam} h\left(A_{i}\right)\right)^{2} \\
\leq C p \sum_{i} m\left(B\left(h\left(x_{i}\right), C_{2} \operatorname{diam} h\left(A_{i}\right)\right)\right) \\
\leq C p m\left(h\left(\pi_{z_{0}}^{-1}\left(B(\sigma, C r) \cap S\left(z_{0}, 1\right)\right)\right)\right) .
\end{gathered}
$$

For Lebesgue almost every $\sigma \in \mathbb{S}^{1}$ there exists $C=C(\sigma)$ so that

$$
\frac{m\left(h\left(\pi_{z_{0}}^{-1}\left(B(\sigma, R) \cap S\left(z_{0}, 1\right)\right)\right)\right)}{R} \leq C,
$$

provided that $R$ is small. Then

$$
\sum_{i} \operatorname{diam} h\left(A_{i}\right) \leq C p r \leq C\left(m_{1}(F)\right)^{1 / 2},
$$

which finishes the proof of the theorem.

\section{Rigidity TheOREM}

Let $f$ be a generalized polynomial-like map such that diam $C_{n} \rightarrow_{n \rightarrow \infty} 0$. Let $V_{i}, i \leq N=N(n)$, be the puzzle pieces at level $n$ and let $U_{j}, j \leq N(n+1)-1$, be the puzzle pieces at level $n+1$ which do not contain the critical point. Define the map $g_{n}: \bigcup_{i} U_{i} \rightarrow \bigcup_{j} V_{j}$, as the map $f$ restricted to $\bigcup_{i} U_{i}$. Let $K\left(g_{n}\right)$ be the filled-in Julia set of $g_{n}$. We can provide each connected component of the Riemann surface $\mathcal{V}=\bigcup_{j} V_{j}$ with its hyperbolic metric. The following result is trivial:

Proposition 4.1. There exist $\alpha_{2}>\alpha_{1}>1$, with $\alpha_{j}=\alpha_{j}(n)$ such that, for any $x \in K\left(g_{n}\right)$, we have the folllowing.

- $\alpha_{1} \leq\|D f(x)\|_{\mathcal{V}} \leq \alpha_{2}$.

- For any $m>0$, let $j$ be so that $f^{m}(x) \in V_{j}$. Then there is a domain $D(x, m)$ which contains $x$ so that $f^{m}$ is univalent in $D(x, m)$ and $f^{m}(D(x, m))=V_{j}$.

The proof of the following corollary is straightforward:

Corollary 4.1. For $n>0$, there exists a centered Markov family $\mathcal{G}_{n}$ so that

- $\mathcal{G}_{n}$ covers $K\left(g_{n}\right)$ in all scales in a $\left(C_{1}, \epsilon_{0}\right)$-uniform way, where $\left(C_{1}, \epsilon_{0}\right)$ depends on $f$ and $n$; 
- $\mathcal{G}_{n}$ has $C_{2}(f, n)$-uniform bounded geometry.

Furthermore, this centered Markov family is topologically defined in the following sense: Let $f_{j}: \bigcup_{i} U_{i}^{j} \rightarrow V^{j}, j=1,2$, be two generalized polynomial-like maps and let $h: \mathbb{C} \rightarrow \mathbb{C}$ be a homeomorphism so that

- $h\left(U_{i}^{1}\right)=U_{i}^{2}$;

- $h\left(V^{1}\right)=V^{2}$;

- $h$ is a conjugacy between $f_{1}$ and $f_{2}$.

Then $h\left(\mathcal{G}_{n}^{1}\right)=\mathcal{G}_{n}^{2}$.

Proof. Let $x \in K\left(g_{n}\right)$. Then $(A, x) \in \mathcal{G}_{n}$ if and only if there exists $m \geq 0$ so that $g_{n}^{m}(x) \in U_{i}$ and $A=D(x, m) \cap g_{n}^{-m}\left(U_{i}\right)$.

Corollary 4.2. The set $\bigcup_{n} K\left(g_{n}\right)$ has zero 2-dimensional Lebesgue measure.

Corollary 4.3. $K(f)$ has empty interior.

Proof. Suppose $K(f)$ has non-empty interior. By the non-existence of wandering components (Sullivan) and the classification of the periodic components, the critical set must be in the interior of $K(f)$, which is a contradiction, since diam $C_{n} \rightarrow 0$.

The following is an argument of Levin and van Strien (Proposition 3.1 in [LvS]):

Proposition 4.2. Let $f: \bigcup_{i} U_{i} \rightarrow V$ be a generalized polynomial-like map so that

- $\operatorname{diam} C_{n} \rightarrow_{n} 0$;

- there exist $M>0$ and a subsequence of critical pieces $C_{n_{k}}$ which admit annuli $A_{k}$ so that

- the internal boundary of $A_{k}$ is $\partial C_{n_{k}}$;

$-\bmod A_{k} \geq M$

- for any $x$ which is not contained in $C_{n_{k}}$ and $m>0$ minimal so that $f^{m}(x) \in C_{n_{k}}$, there exists a neighborhood $V(x, m)$ of $x$ so that

* $f^{m}(V(x, m))=C_{n_{k}} \cup A_{k}$;

* $f^{m}$ is univalent in $V(x, m)$.

Then any point $x \in K(f)$ admits a sequence of puzzle pieces which shrinks to $x$. In particular, $K(f)$ is a Cantor set.

Proof. By Proposition 4.1, we can assume that the forward orbit of $x$ accumulates on the critical point. Let $m_{k}$ be minimal so that $f^{m_{k}}(x) \in C_{n_{k}}$. Since $m_{k}$ is the first entry time, there exists a domain $V(x, k)$ containing $x$ which is mapped univalently by $f^{m_{k}}$ to $C_{n_{k}} \cup A_{k}$. Denote by $P(x, k) \subset V(x, k)$ the piece such that $f^{m_{k}} P(x, k)=C_{n_{k}}$. We claim that diam $P(x, k)$ goes to zero. Indeed, consider the core curve $\gamma_{k}$ of the annulus $A_{k}$. Since $\bmod A_{k}>M$ and by the Koebe distortion lemma, we have that $\tilde{\gamma}_{k}=f^{-m_{k}}\left(\gamma_{k}\right) \cap V(x, k)$ is a curve which contains a disc whose diameter is comparable to diam $\tilde{\gamma}_{k}$ and whose center is $x$. So, if diam $\tilde{\gamma}_{k}$ does not converge to zero, the diameter of these balls also does not converge to 0 , and we can find a subsequence converging to a ball which is contained in $K(f)$, since $f^{m}, m>0$, is a normal family in this ball. This is impossible, since int $K(f)$ is empty.

Proof of Theorem 1. Since the argument is quite symmetric with respect to $f_{1}$ and $f_{2}$, we will denote both by $f$. Decompose $K(f)$ into two disjoint sets $K(f)=$ $X_{1} \cup X_{2}$, where $X_{2}=\bigcup_{n} K\left(g_{n}\right)$. Denote $E_{n}=K\left(g_{n}\right)-K\left(g_{n-1}\right)$, with $K\left(g_{0}\right):=\phi$. Then $X_{2}=\bigcup_{n} E_{n}$. Define the centered Markov family $\mathcal{F}_{2}$ in the following way: if 
$x \in E_{n}$, then the domains in $\mathcal{F}_{2}$ with center $x$ are exactly the domains with center $x$ belonging to $\mathcal{G}_{n}$. It is easy to see that $\mathcal{F}_{2}$ is topologically defined, covers $X_{2}$ in all scales and has bounded geometry.

Now we will deal with $X_{1}$ : this is the set of points in $K(f)$ whose positive orbit accumulates on the critical point. Define the Markov family $\mathcal{F}_{1}$ in the following way: for $x \in K_{1}$, let $m_{k} \geq 0$ be minimal so that $f^{m_{k}}(x) \in C_{n_{k}}$. By assumption, there is a domain which contains $x$ such that $f^{m_{k}}$ maps it univalently to $C_{n_{k}} \cup A_{k}$. So there exists a puzzle piece $V(x, n)$ containing $x$ so that

- $f^{m_{k}}$ is univalent in $V(x, n)$;

- $f^{m_{k}}(V(x, n))=C_{n_{k}}$

- moreover, since $\bmod A_{k}>M$ and by the Koebe distortion lemma, there exists $\lambda<1$ so that for any $(x, n)$ there is $y \in V(x, n)$ satisfying

$$
B(y, \lambda V(x, n)) \subset V(x, n)
$$

where $\lambda=\lambda\left(M, K_{1}, K_{2}\right)$.

Note that, for a fixed $x$, diam $V(x, n) \rightarrow_{n} 0$. Define

$$
\mathcal{F}_{1}=\left\{(V(x, n), x): x \in X_{1} \text { and } n>0\right\} .
$$

So $\mathcal{F}_{1}$ covers $X_{1}$ in arbitrary small scales, has bounded geometry and, moreover, is topologically defined. By the Removability Theorem (Theorem 44), $h$ is quasiconformal in $\mathbb{C}$.

\section{Part 2. Characterization of the stable manifold of the Fibonacci cycle}

\section{Preliminaries}

5.1. Quasiconformal theory. We will assume that the reader is familiar with the general properties of quasiconformal maps (Lehto and Virtanen [LV] is a good reference) and quasiconformal vector fields (see $[\mathrm{McM}]$ ). The following property will be very useful:

Proposition 5.1 ( $(\mathrm{McM}])$. Let $z_{0}, z_{1}$ and $z_{2}$ be distinct points on the Riemann sphere and let $K>0$. Then the set of $K$-quasiconformal vector fields on the Riemann sphere which vanishes at these points is a compact set in the space of continuous vector fields on the Riemann sphere with the sup norm induced by the spherical metric.

5.2. Banach spaces. We will denote domains (pre-compact open sets in the complex plane) with symbols like $U, V, \tilde{V}, \hat{U}, \ldots$ If we denote a domain as, for instance, $U=U_{1} \cup \cdots \cup U_{n}$, the reader must assume that $U_{i}$ are the (simply) connected components of $U$. If $\alpha \in \mathbb{C}$, then $\alpha U:=\{\alpha \cdot x: x \in U\}$. The $\delta$-neighborhood of a set $X$, denoted $\delta-X$, is the set $\{x: \operatorname{dist}(x, X) \leq \delta \operatorname{diam} X\}$. Let $U$ be a domain so that $\bar{U}$ does not disconnect the plane. Let $d>1$. In the Banach space $\mathcal{B}(U)$ of holomorphic functions on $U$ with continuous extension to $\bar{U}$, equipped with the sup norm, consider the closed affine space $\mathcal{B}_{\text {nor }}(U)$ of functions $f$ such that

- the domain $U$ contains 1 and 0 and furthermore $f(1)=1$ and $f^{(i)}(0)=0$, for $1 \leq i<d$. 
We will call $\mathcal{B}_{\text {nor }}(U)$ a Banach slice. The tangent space of $\mathcal{B}_{\text {nor }}$, denoted by $T \mathcal{B}_{\text {nor }}(U)$, is the subspace of the vectors $v \in \mathcal{B}(U)$ such that

- $v(1)=0$,

- $v^{(i)}(0)=0$, for $1 \leq i<d$.

Let $f: U_{0} \cup \cdots \cup U_{n} \rightarrow V$, where $U_{i}$ are simply connected, be a generalized polynomial-like map and let $\tilde{U}=\tilde{U}_{0} \cup \cdots \cup \tilde{U}_{n}$, where $U_{i} \Subset \tilde{U}_{i}$ are simply connected. Then we say that the Banach space $\mathcal{B}_{\text {nor }}(\tilde{U})$ is compatible with $f$. By a result of Levin and van Strien (Proposition 2.2 in [LvS]), if $g: \hat{U}_{1} \cup \cdots \cup \hat{U}_{n} \rightarrow \hat{V}$ is a generalized polynomial-like restriction of $f$, with $\hat{U}_{i} \subset \tilde{U}_{i}$, then $K(g)=K(f)$. We will say that $g$ is a representation of $f$ subordinated to $\tilde{U}$. So all these representations have the same filled-in Julia set and, if the critical points of $f$ do not escape, they have the same postcritical set.

5.3. The principal nest. Let $f: \bigcup_{i} U_{i} \rightarrow V$ be a generalized polynomial-like map whose critical point does not escape. Denote $V_{0}^{0}:=V, V_{0}^{1}:=U_{0}$ and $V_{1}^{1}:=U_{k}$, where the critical point is in $U_{0}$ and the critical value is in $U_{k}$. Denote $f_{0}:=f$. The map $f$ restricted to $V_{0}^{1}$ will be denoted $f_{0}^{0}$ and $f$ restricted to $V_{1}^{1}$ by $f_{0}^{1}$. Denote $\mathcal{E}_{0}:=\phi$ and $\mathcal{D}_{0}:=\left\{V_{0}^{1}, V_{1}^{1}\right\}$. Suppose, by induction, that we have defined $V_{0}^{n}$, with $0 \in V_{0}^{n}$ and the following property:

$$
f^{i}\left(\partial V_{0}^{n}\right) \cap V_{0}^{n}=\phi,
$$

for every $i>0$ so that $f^{i}$ is defined on $V_{0}^{n}$. Denote by $\mathcal{E}_{n}$ the family of (simply) connected components of

$$
\left\{x \in \bigcup_{i} U_{i}: \text { there exists } m \geq 0 \text { so that } f^{m}(x) \in V_{0}^{n}\right\} .
$$

The first entry map to $V_{0}^{n}$ of a point $x \in \bigcup \mathcal{E}_{n}$, denoted by $\pi_{n}(x)$, is defined as $f^{m}(x)$, where $m \geq 0$ is minimal so that $f^{m}(x) \in V_{0}^{n}$. The first return map to $V_{0}^{n}$, denoted by $f_{n}$, is defined as $\pi_{n} \circ f$ in $\bigcup \mathcal{D}_{n}$, where $\mathcal{D}_{n}$ is the family of (simply) connected components of $f^{-1} E$, where $E \in \mathcal{E}_{n}$. Note that $\mathcal{E}_{n}-\left\{V_{0}^{n}\right\} \subset \mathcal{D}_{n}$. Denote by $V_{0}^{n+1} \in \mathcal{D}_{n}$ the domain which contains the critical point and by $V_{1}^{n+1} \in \mathcal{D}_{n}$ the domain which contains $f_{n}(0)$. Note that $V_{0}^{n}$ is a nested sequence of critical puzzle pieces, called the principal nest.

\section{On the MAPS IN THE FibONACCI CYCLE}

The most important property of the sequence of critical puzzle pieces in the principal nest of a map in the the Fibonacci cycle is that their shape is under control, as we will describe below. It is important to note that the figures in this work are only illustrative: the actual shape of the puzzles and Julia sets depends on the criticality $d$ and they are much more complicated. Taking the figures too literally, the reader might deduce that $f_{n}^{0}$ has degree two, which is not the case. The actual domain $D_{0}$ represented in Figure 4, for instance, has more holes (which do not intersect the real line). Realistic pictures of puzzle pieces of Fibonacci maps can be found in Buff $[\mathrm{Bu}$. 


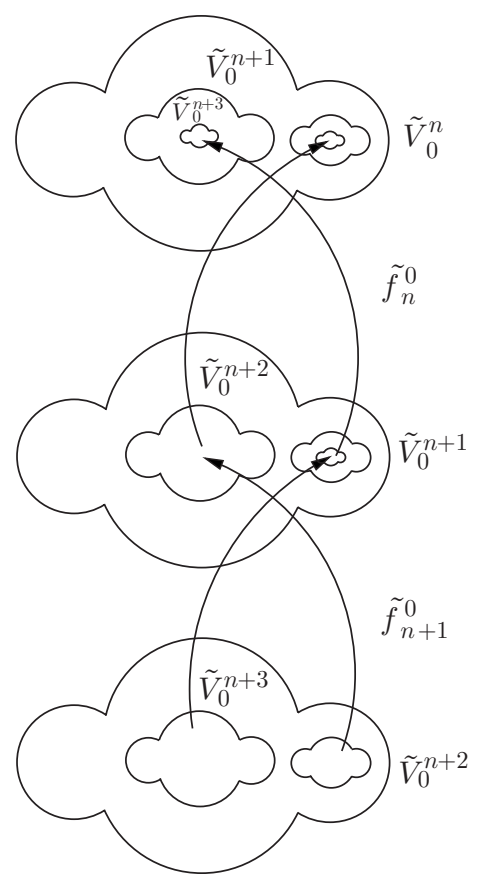

FIGURE 2.

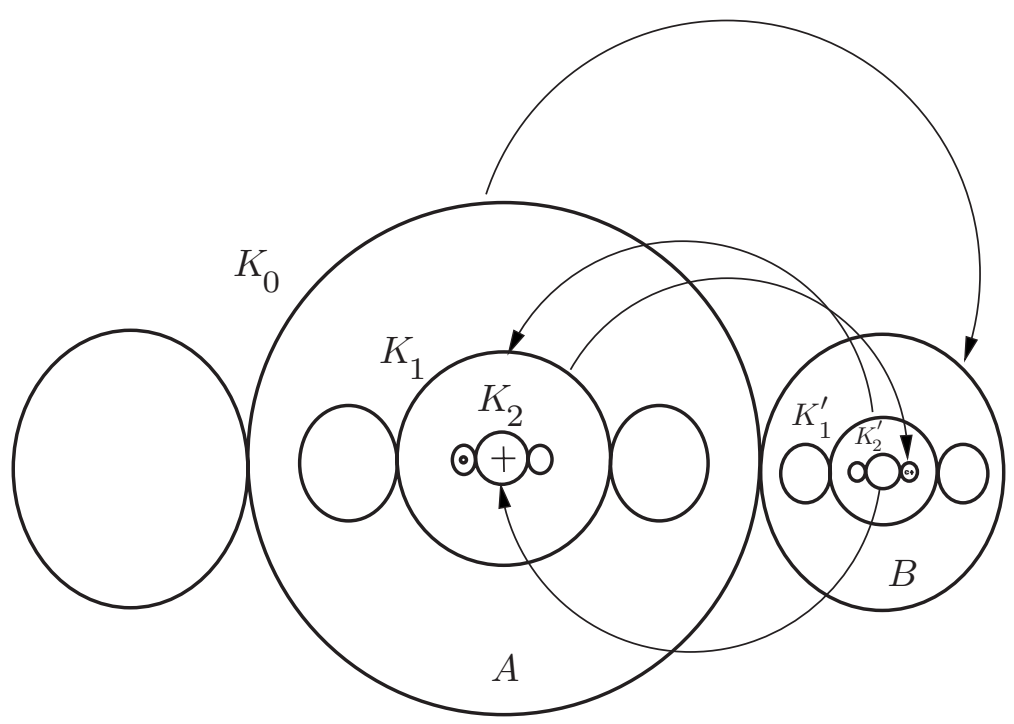

FiguRE 3.

Figure 2 describes how certain puzzles pieces in real Fibonacci maps are permuted by the action of the generalized renormalizations. Buff $[\mathrm{Bu}]$ described precisely the shape of $V_{0}^{n}$. Denote $d_{H}\left(T_{1}, T_{2}\right):=\max \left\{d_{h}\left(\bar{T}_{1}, \bar{T}_{2}\right), d_{h}\left(\partial T_{1}, \partial T_{2}\right)\right\}$, where $d_{h}(\cdot, \cdot)$ represents the Hausdorff metric on the set of compact sets. 


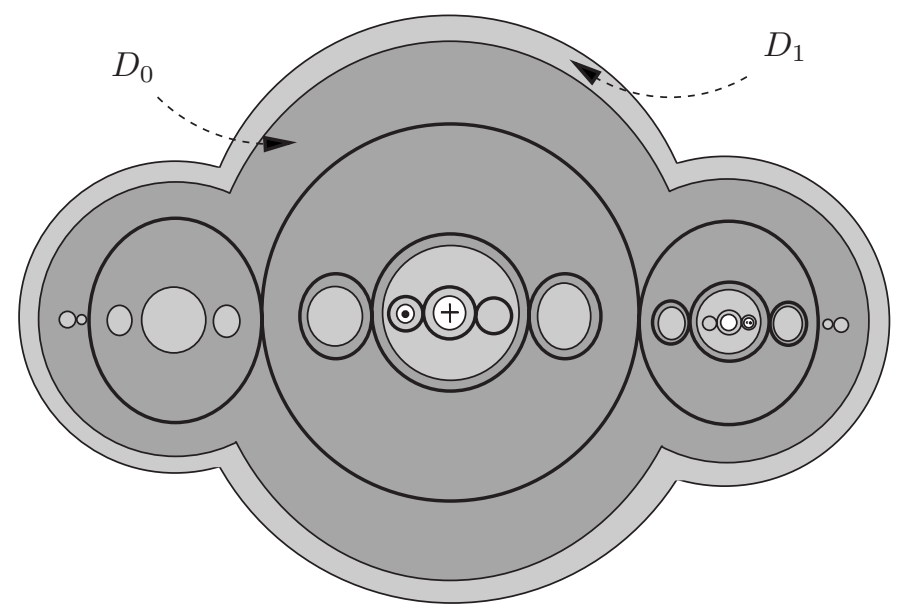

FiguRE 4.

Proposition 6.1 ( $(\overline{\mathrm{Bu}})$. Let $\beta \in(0,1)$ be the closest point with period two to the critical point of $\tilde{f}$. Then there exists a polynomial-like map $g$, with a unique critical point and a hyperbolic attractor of period two so that

$$
\begin{gathered}
-1 / \beta^{2} g^{2}(\beta z)=g(z), \\
\tilde{f}_{0}^{0}(z)=g\left(\frac{1}{\beta} \cdot z\right),
\end{gathered}
$$

and

$$
\tilde{f}_{0}^{1}(z)=\mp \frac{1}{\beta} g(z) .
$$

In particular $g(z)=1 / \beta^{n} \tilde{f}_{n}^{0}\left(\beta^{n+1} \cdot z\right)$. Furthermore

$$
\operatorname{dist}_{H}\left(\frac{1}{\beta^{n}} \tilde{V}_{0}^{n}, K(g)\right) \rightarrow_{n} 0 .
$$

Indeed, Buff claimed that $1 / \beta^{n} \overline{\tilde{V}_{0}^{n}}$ converges to $K(g)$ in the Hausdorff metric on compact sets, but the slight modification above can be proved in an analogous way (indeed, we will do something similar later, in the proof of Proposition 8.11). We will normalize $\tilde{f}$ so that 0 is a maximum and 1 is the fixed point of $\tilde{f}$ in the central domain so that $D \tilde{f}(1)<0$. Then the corresponding fixed point of $\mathcal{R} \tilde{f}$ is $\beta$ (or $-\beta$ ).

Denote by $A$ the connected component of the interior of $K_{0}:=K(g)$ containing the critical point and by $B$ the connected component that contains the critical value. We will say that a set $O_{1}$ is compactly contained in $O_{2}$, denoted $O_{1} \Subset O_{2}$, if $\bar{O}_{1} \subset$ int $O_{2}$.

The following result will be very important:

Proposition 6.2 ([B] $)$. Denote $K_{1}:=\beta K_{0}, K_{2}:=\beta^{2} K_{0}, K_{i}^{\prime}:=g^{-1} K_{i} \cap B$. Then

- $-1 / \beta^{2} g^{2}(\beta z)=g(z)$,

- $K_{1} \subset A$,

- $K_{2} \Subset K_{1}$,

- $g\left(K_{1}\right)=K_{2}^{\prime}$. 
In particular:

- $\tilde{f}^{0}\left(K_{1}\right)=K_{0}$

- $\tilde{f}^{1}\left(K_{1}^{\prime}\right)=K_{0}$

- $\tilde{f}^{1}\left(K_{2}^{\prime}\right)=K_{1}$.

Proof. These items are included in, or are an easy consequence of, Buff $[\mathrm{Bu}]$.

Figure 3 describes how $g$ acts on $K_{0}, K_{1}, K_{0}^{\prime}$ and $K_{1}^{\prime}$ : these sets are not represented in the figure, but only the (boundaries of the) connected components (in the figure, circles) of their interior which correspond to the topological discs $A, B$ and $-B$ contained in $K(g)$.

Note that $1 / \beta^{n} \partial \tilde{V}_{0}^{n}$ converges to $\partial K_{0}, 1 / \beta^{n} \partial \tilde{V}_{0}^{n+1}$ converges to $\partial K_{1}$ and $1 / \beta^{n} \partial \tilde{V}_{1}^{n+1}$ converges to $\partial K_{1}^{\prime}$. Since $P(\tilde{f}) \cap\left(\tilde{V}_{0}^{n+1}-\left(\tilde{V}_{0}^{n+1} \cup \tilde{V}_{1}^{n}\right)\right)=\phi$, we conclude that $\tilde{f}$ satisfies all the conditions imposed on $f_{1}$ in Theorem 1, with $C_{i_{n}}:=V_{0}^{n}$.

The following corollary will be a useful tool in Section 9 .

Corollary 6.1. There exists an open set $D_{1}$ which contains the Julia set $J(g)$ in its interior such that, if we denote $D_{0}:=g^{-2} D_{1}$, then

- $g^{2}: D_{0} \rightarrow D_{1}$ is an unramified covering of degree $(\text { deg } g)^{2}$,

- $D_{0} \Subset D_{1}$,

- $\beta D_{0} \Subset D_{1}$,

- $g^{-1}\left(\beta D_{0}\right) \cap B \Subset D_{1}$.

Proof. Let $A_{1} \Subset A$ and $B_{1} \Subset B$ be domains whose boundaries are Jordan curves very close to $J(g)$ and such that

- $B_{1} \Subset g^{-1} A_{1}$,

- $A_{1} \Subset g^{-1} B_{1}$.

Let $U$ be a small neighborhood of $K(f)$ so that $g^{-1} U \Subset U$. We define $D_{1}$ as (see Figure 4)

$$
D_{1}:=U \backslash\left(\beta^{2} \bar{A}_{1} \cup \beta^{2} \bar{B}_{1} \cup\left(g^{-1}\left(\beta^{2} \bar{B}_{1}\right) \cap B\right) \cup\left(g^{-1}\left(\beta^{2} \bar{A}_{1}\right) \cap B\right)\right) .
$$

Note that

$$
D_{0}:=g^{-2} D_{1} \Subset g^{-1} U \backslash\left(\beta g^{-1} \bar{A}_{1} \cup \beta g^{-1} \bar{B}_{1} \cup g^{-1}\left(\beta g^{-1} \bar{A}_{1} \cup \beta g^{-1} \bar{B}_{1}\right)\right) \subset D_{1} .
$$

The other properties of $D_{1}$ are immediate consequence of the previous proposition (provided we have selected $\partial A_{1}, \partial B_{1}$ and $\partial U$ close to $J(g)$ ).

\section{The FibonacCi Renormalization operator: COMPLEX ANALYTIC VERSION}

We are going to define the complex analytic version of the Fibonacci operator, defined in a convenient Banach slice. Let $\tilde{f}$ be one of the maps in the Fibonacci cycle. By Proposition 6.2, the $\delta$-neighborhoods of $K_{2}, \tilde{f}\left(K_{2}\right)$ and $\beta K_{1}^{\prime}$ are contained in the interior of $K_{1} \cup K_{1}^{\prime}$, for some $\delta>0$. Recall that we normalized $\tilde{f}$ so that $\beta$ (or $-\beta$ ) is a fixed point of $\tilde{f}^{2}$. Taking $\epsilon_{0}$ small enough, we can assume that $\beta$ (or $-\beta$ ) has an analytic continuation, denoted $\beta_{f}$, for every $f$ so that $|f-\tilde{f}|_{\mathcal{B}\left(K_{1} \cup K_{1}^{\prime}\right)} \leq \epsilon_{0}$. The above considerations imply that there exist $\epsilon_{0}>0$ and $C<1$ so that if $f \in \mathcal{B}_{\text {nor }}\left(K_{1} \cup K_{1}^{\prime}\right)$ and $|f-\tilde{f}|_{\mathcal{B}\left(K_{1} \cup K_{1}^{\prime}\right)} \leq \epsilon_{0}$, then $f^{2}$ is well defined in a $C \delta$ neighborhood of $\beta_{f} K_{1}$ and $f$ is defined in a $C \delta$-neighborhood of $\beta_{f} K_{1}^{\prime}$. We will select a domain which contains $K_{1} \cup K_{1}^{\prime}$ and whose boundary is more regular: 
Let $U_{0}$ and $U_{1}$ be simply connected domains so that $K_{1} \Subset U_{0} \subset C \delta / 2-K_{1}$ and $K_{1}^{\prime} \Subset U_{1} \subset C \delta / 2-K_{1}^{\prime}$ and whose boundaries are analytic curves. So for every $f \in \mathcal{B}_{\text {nor }}\left(U_{0} \cup U_{1}\right)$ satisfying $|f-\tilde{f}|_{\mathcal{B}\left(U_{0} \cup U_{1}\right)} \leq \epsilon_{0}, f^{2}$ is defined in a $2 C \delta / 3$ neighborhood of $\beta_{f} \bar{U}_{0}$ and $f$ is defined in a $2 C \delta / 3$-neighborhood of $\beta_{f} \bar{U}_{1}$. Define the map $\hat{\mathcal{R}} f \in \mathcal{B}_{\text {nor }}\left(2 C \delta / 3-\bar{U}_{0} \cup 2 C \delta / 3-\bar{U}_{1}\right)$ as

$$
\frac{1}{\beta_{f}} f^{2}\left(\beta_{f} \cdot z\right)
$$

on $2 C \delta / 3-\bar{U}_{0}$ and

$$
\frac{1}{\beta_{f}} f\left(\beta_{f} \cdot z\right)
$$

on $2 C \delta / 3-\bar{U}_{1}$. The Fibonacci renormalization operator is defined as $\mathcal{R}:=i \circ \hat{\mathcal{R}}$, where $i: \mathcal{B}_{\text {nor }}\left(2 C \delta / 3-\bar{U}_{0} \cup 2 C \delta / 3-\bar{U}_{1}\right) \rightarrow \mathcal{B}_{\text {nor }}\left(U_{0} \cup U_{1}\right)$ is the inclusion $i(f)=f$. This operator is an example of an induced transformation, whose properties will be described in Section 11. In the rest of this work, denote $U:=U_{1} \cup U_{2}$. Of course, using the complex bounds result for real Fibonacci maps vSN94, for a Banach slice $\mathcal{B}_{\text {nor }}(\hat{U})$ we can define a complex analytic version of iteration of the Fibonacci renormalization operator in an easier way, without using the special features of $\tilde{f}$, and carry out a proof of the hyperbolicity in this Banach space as well. The problem is that the exposition would be a little more complicated, especially because we need to deal with an iterate of the Fibonacci operator (not with the operator itself) and there is no guarantee in this approach that $K_{1} \cup K_{1}^{\prime} \subset \hat{U}$ : so there is no advantage in using this approach in the case of the Fibonacci renormalization (but this alternative method is, possibly, the unique way to define the complex extension in the case of generalizations of this work for other combinatorics, such as non-periodic ones).

\section{Control of the GeOMEtry In the PRINCIPAL NEST}

Let $f$ be a function in $\mathcal{B}_{\text {nor }}(U)$ such that $\mathcal{R}^{n} f$ exists and

$$
\left|\mathcal{R}^{n} f-\tilde{f}\right|_{\mathcal{B}(U)} \leq \delta
$$

for all $n \geq 0$. We will consider a generalized polynomial-like restriction of $f$ and prove that the shape of the puzzle pieces in it principal nest is under control, provided that $\delta$ is small. Indeed we prove that the shapes of the critical puzzle pieces are close to the shape of the Julia set of the polynomial-like map $g$ defined in Section 6. The fact that $\mathrm{g}$ is a hyperbolic polynomial-like map is crucial in this result.

Let $D \Subset g(D) \subset V$ be a simply connected domain, which contains $K(g)$ so that $g: D \rightarrow g(D)$ is a ramified covering map and let $D_{a} \subset A, D_{b} \subset B$, with $\partial D_{a} \cup \partial D_{b}$ very close to $J(g)$, be simply connected domains so that $g\left(D_{a}\right) \Subset D_{b}$ and $g\left(D_{b}\right) \Subset D_{a}$. Define $E_{0}:=D \backslash \overline{D_{a}} \cup \overline{D_{b}}$ and $E_{n}:=g^{-n} E_{0}$. Then

$$
g: E_{n+1} \rightarrow E_{n}
$$

is a covering map and, because $g$ is a hyperbolic map, if we take a Jordan curve $\gamma \subset E_{0}$ that is the boundary of a bounded domain containing the postcritical set (and the attractor) of $g$, then $g^{-n} \gamma$ is also a Jordan curve bounding a domain which contains the postcritical set of $g$ and $d_{H}\left(g^{-n} \gamma, J(g)\right) \leq C \lambda^{n}$, for some $\lambda<$

1. (Recall that $d_{H}\left(T_{1}, T_{2}\right)$ denotes $\max \left\{d_{h}\left(\bar{T}_{1}, \bar{T}_{2}\right), d_{h}\left(\partial T_{1}, \partial T_{2}\right)\right\}$, where $d_{h}(\cdot, \cdot)$ 


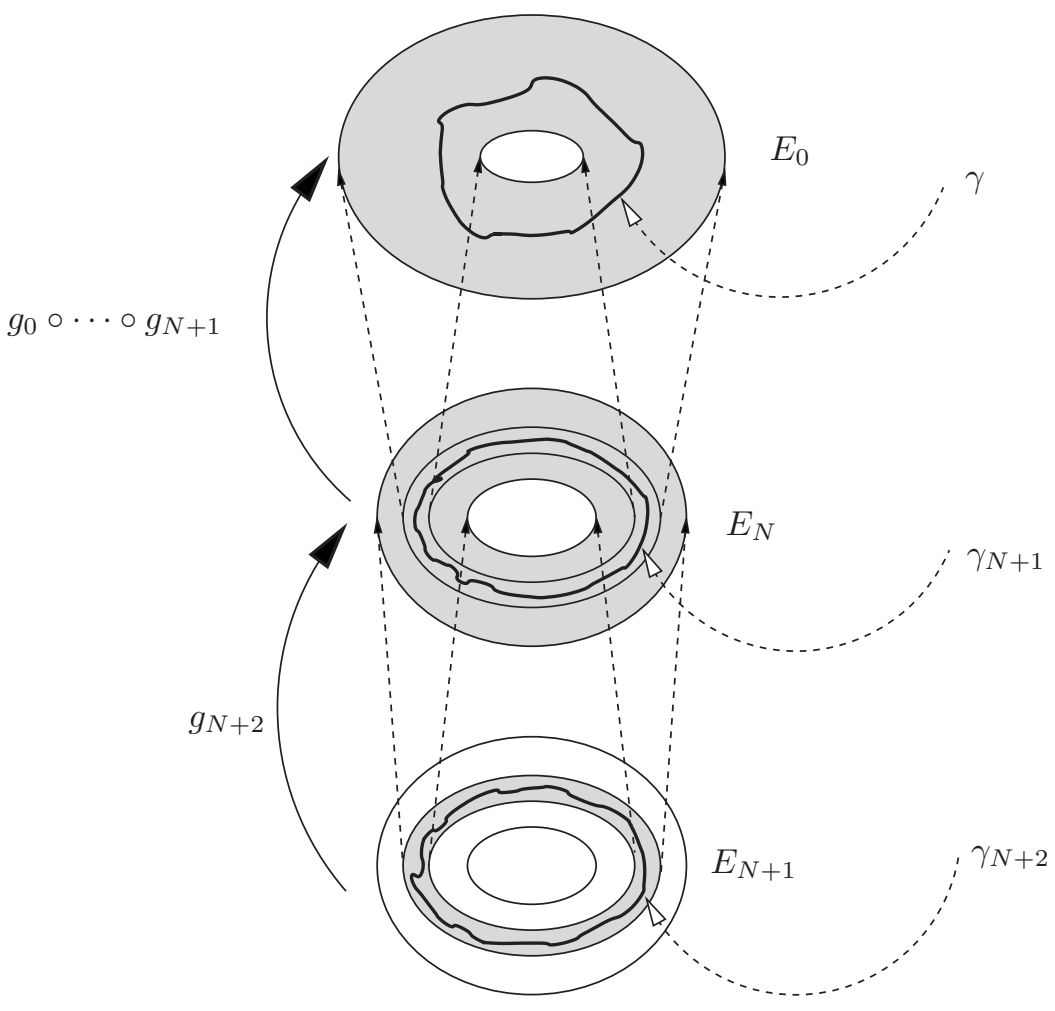

FiguRE 5.

represents the Hausdorff metric under the set of compact sets.) Consider an analytic curve $\gamma \subset E_{0}$, as above, and denote by $V_{0}^{0}$ the simply connected bounded domain whose boundary is $\gamma$. Denote $\tilde{\tau}_{n}=\beta^{n}$ and $\beta_{i}:=\beta_{\mathcal{R}^{i} f}$, and $\tau_{n}:=\beta_{n-1} \cdots \beta_{0}$. This notation will be used in the rest of this work.

Proposition 8.1. For every $\epsilon>0$ there exist $\delta>0$ and $N=N(\epsilon)$ with the following properties: If $f \in \mathcal{B}_{\text {nor }}(U)$ satisfies

$$
\left|\mathcal{R}^{n} f-\tilde{f}\right| \leq \delta
$$

for $n \geq 0$, and $V_{0}^{1}$ and $V_{1}^{1}$ are the connected components of $f^{-1} V_{0}^{0}$ which contain the critical point and $f(0)$, then $f: V_{0}^{1} \cup V_{1}^{1} \rightarrow V_{0}^{0}$ is a generalized polynomial-like map so that

(1) the critical point does not escape;

(2) using the notation defined in Section [5.3, $V_{0}^{n+1} \neq V_{1}^{n+1}$ and $P(f) \cap V_{0}^{n} \subset$ $V_{0}^{n+1} \cup V_{1}^{n+1}$

(3) $f_{n}^{0}: V_{0}^{n+1} \rightarrow V_{0}^{n}$ is a restriction of $f^{S_{n}}$ to $\tau_{n} U_{0}$ and $f_{n}^{1}: V_{1}^{n+1} \rightarrow V_{0}^{n}$ is a restriction of $f^{S_{n-1}}$ to $\tau_{n} U_{1}$; 
(4) we have

$$
\begin{gathered}
\operatorname{dist}_{H}\left(\frac{1}{\tau_{n}} V_{0}^{n}, K_{0}\right) \leq \epsilon, \\
\operatorname{dist}_{H}\left(\frac{1}{\tau_{n}} V_{0}^{n+1}, K_{1}\right) \leq \epsilon, \\
\operatorname{dist}_{H}\left(\frac{1}{\tau_{n}} V_{1}^{n+1}, K_{1}^{\prime}\right) \leq \epsilon, \\
\operatorname{dist}_{H}\left(\frac{1}{\tau_{n}}\left(f_{n}^{1}\right)^{-1}\left(V_{0}^{n+1}\right), K_{2}^{\prime}\right) \leq \epsilon,
\end{gathered}
$$

for $n \geq N$.

Furthermore, there exists $C>0$ such that

$$
\operatorname{dist}\left(\frac{1}{\tau_{n}} f_{n}^{0}(0), \frac{1}{\tau_{n}} \partial\left(f_{n}^{1}\right)^{-1}\left(V_{0}^{n+1}\right)\right) \geq C .
$$

In particular, all the assumptions about the generalized polynomial-like maps in Theorem 1 are satisfied by $f$ and, if $\epsilon$ is small enough (see Figure 6),

- $V_{0}^{n-2} \backslash V_{0}^{n-1} \cup V_{1}^{n-1} \Subset \tau_{n-2} D_{1}$,

- $D_{0}^{n-2} \Subset \tau_{n-2} D_{1}$,

- $\left(f_{n-2}^{1}\right)^{-1}\left(\tau_{n-2} D_{0}^{n-2}\right) \Subset \tau_{n-2} D_{1}$,

- $\tau_{n-1} D_{0}^{n-1} \Subset \tau_{n-2} D_{1}$,

where $D_{0}^{n}:=\tau_{n+1}^{-1}\left(f_{n}^{0}\right)^{-1} \tau_{n} D_{1}$.

Proof. Note that by definition $\mathcal{R}^{n} f$ is an appropriate normalization of $\left(\mathcal{R}^{n-1} f\right)^{2}$ on $U_{0}$ and it is a normalized restriction of $\mathcal{R}^{n-1} f$ on $U_{1}$. So it is easy to conclude inductively that

$$
f^{S_{n}}(z)=\tau_{n} \mathcal{R}^{n} f\left(\tau_{n}^{-1} z\right) \text { on } \tau_{n} U_{0}
$$

and

$$
f^{S_{n-1}}(z)=\tau_{n} \mathcal{R}^{n} f\left(\tau_{n}^{-1} z\right) \text { on } \tau_{n} U_{1} .
$$

Recall that $\tilde{f}(\beta z)=g(z)$ on $U_{0}$. Since the renormalizations of $f$ are very close to $\tilde{f}, g_{n}(z):=\mathcal{R}^{n} f\left(\beta_{n} z\right)$ are very close to $g(z)$ on $U_{0}$. Consider $N$ so that $\operatorname{dist}_{H}\left(E_{N}, J(g)\right) \leq \epsilon / 2$. Choosing $\delta$ small enough, we can assume that

$$
\left(g_{N+1} \circ \cdots \circ g_{0}\right)^{-1} E_{0} \Subset E_{N+1},
$$

and, for $n \geq 0$,

$$
\left(g_{n}\right)^{-1} E_{N} \Subset E_{N} .
$$

Figure 5 illustrates this, but the topology and shape of the sets $E_{i}$ are not correct in the figure: $E_{0}$ has two holes, and the number of holes of $E_{i}$ grows exponentially fast with $i$. Since $\gamma:=\partial V_{0}^{0} \subset E_{0}$, by equation (1) and the above properties, we can inductively define Jordan curves $\gamma_{n}$ in the following way: $\gamma_{0}:=\gamma$, and $\gamma_{n}$ is the Jordan curve contained in the set $f^{-S_{n-1}} \gamma_{n-1}$ such that the bounded domain with boundary $\gamma_{n}$, denoted $\hat{V}_{0}^{n}$, contains the critical point. These domains have the following properties:

- $\hat{V}_{0}^{n} \Subset \hat{V}_{0}^{n-1} \subset \tau_{n-1} U_{0}$

- $f^{S_{n}}: \hat{V}_{0}^{n+1} \rightarrow \hat{V}_{0}^{n}$ is a ramified covering with a unique critical point; 

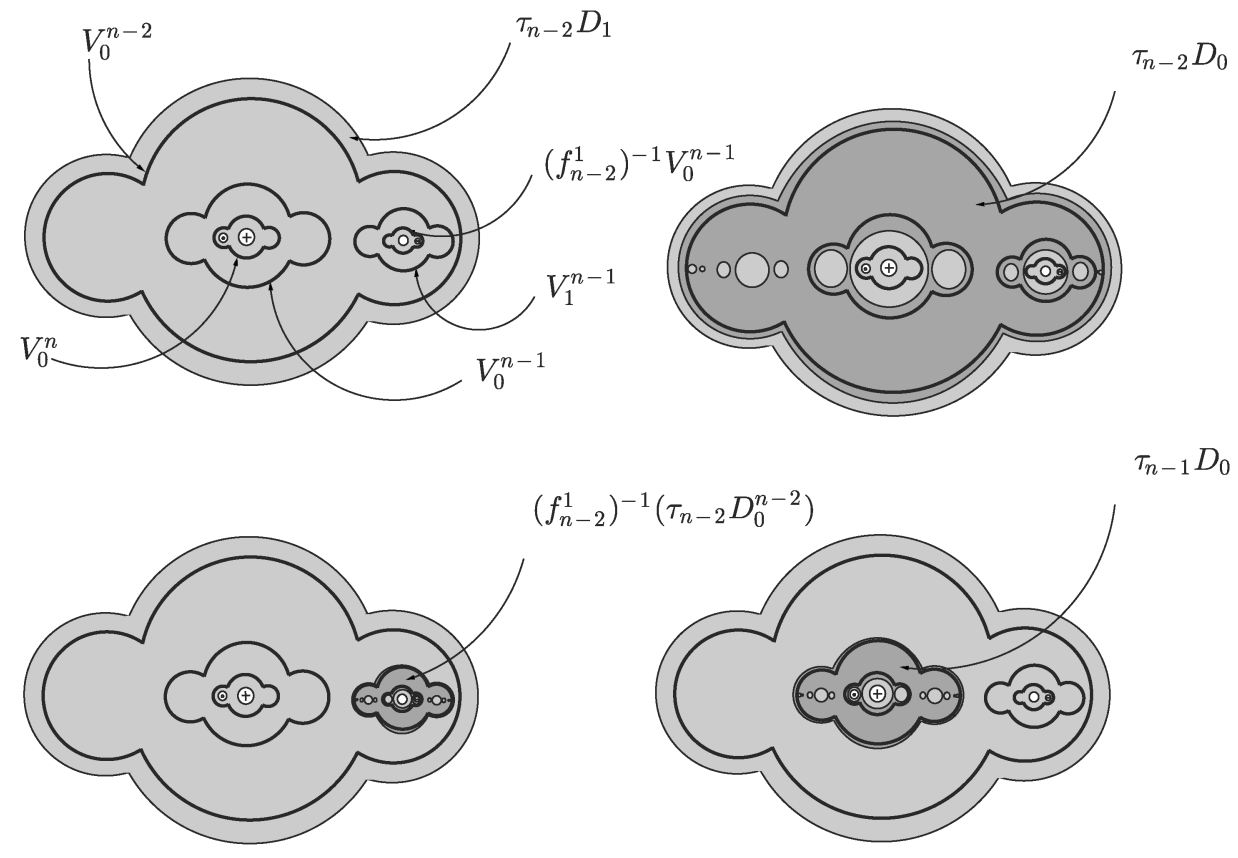

FiguRE 6.

- we have

for $n \geq N$.

In particular, the critical point does not escape. We claim that $\hat{V}_{0}^{n}=V_{0}^{n}$, where $V_{0}^{n}$ were defined in Section 5.3. Suppose, by induction, that we proved the claim for $V_{0}^{i}, i<n$. In particular, the time of the first return of the critical point to $V_{0}^{n-3}$ and $V_{0}^{n-2}$ is $S_{n-3}$ and $S_{n-2}$. By definition, $V_{0}^{n}$ is the domain of the first return map to $V_{0}^{n-1}$ which contains the critical point. So it is sufficient to prove that the time of the first return $m$ of the critical point to $V_{0}^{n-1}$ is $S_{n-1}$. Clearly $m \leq S_{n-1}$. But note that $f^{S_{n-2}}(0) \in V_{0}^{n-2} \backslash V_{0}^{n-1}$, because

$$
f^{S_{n-2}}(0)=\tau_{n-2} \mathcal{R}^{n-2} f(0) \in \tau_{n-2} U_{1},
$$

and

$$
V_{0}^{n-1} \subset \tau_{n-1} U_{0} \subset \tau_{n-2} U_{0} .
$$

So the first chance for the forward orbit of $f^{S_{n-2}}(0)$ to enter $V_{0}^{n-1}$ is its iteration by the time of the first return of $V_{0}^{n-2}$ to $V_{0}^{n-3}$; in other words, $f^{S_{n-2}+S_{n-3}}(0)=$ $f^{S_{n-1}}(0)$. This finishes the proof of the claim. Note that, because $f^{S_{n-1}}(0) \in$ $V_{0}^{n-1} \backslash V_{0}^{n}$, we have that $V_{1}^{n} \neq V_{0}^{n}$, and since $f^{S_{n-1}+S_{n-2}}(0) \in V_{0}^{n}$, by equation (4) we have $V_{1}^{n}=\left(f_{n}^{1}\right)^{-1} V_{0}^{n-1}$, where $f_{n}^{1}$, as defined in Section [5.3, is a restriction of $f^{S_{n-2}}$ on $\tau_{n-1} U_{1}$. By equation (3), and because $\mathcal{R}^{n} f$ is very close to $\tilde{f}$ and $\tau_{n}^{-1} V_{0}^{n}$ is $\epsilon$-close to $K_{0}$, we have

$$
\operatorname{dist}_{H}\left(\tau_{n}^{-1} V_{1}^{n+1}, K_{1}^{\prime}\right) \leq O(\epsilon) .
$$


Fix $n \geq 0$. Note that the map $f_{i}$ on $V_{0}^{i+1} \cup V_{1}^{i+1}$, for $i \geq n$, is a composition of iterations of $f_{n}$ on $V_{0}^{n+1} \cup V_{1}^{n+1}$. This is enough to conclude that the postcritical set of $f$ does not intersect $V_{0}^{i} \backslash\left(V_{0}^{i+1} \cup V_{1}^{i+1}\right)$.

\section{QUASICONFORMAL RIGIDITY}

A map $f \in \mathcal{B}_{\text {nor }}(U)$ satisfying the assumptions in Proposition 8.1 has a dynamical behavior quite similar to the maps in the Fibonacci cycle: the main result in this section is that there exists a hybrid conjugacy between $f$ and either $\tilde{f}_{1}$ or $\tilde{f}_{2}$.

If $A$ is a connected domain omitting at least two points of the complex plane, we can provide it with its hyperbolic metric, denoted $\operatorname{dist}(\cdot, \cdot)_{D}$. Consider a map $f \in \mathcal{B}_{\text {nor }}(U)$ which is very close to $\tilde{f}$. Let $\tilde{V}_{0}=V_{0}^{0}$ be the simply connected domain whose boundary is $\gamma$ (see the previous section). Define $V_{0}^{1}:=\left(f_{0}^{0}\right)^{-1} V_{0}$ and $V_{1}^{1}:=\left(f_{0}^{1}\right)^{-1} V_{0}$ and similarly $\tilde{V}_{0}^{1}$ and $\tilde{V}_{1}^{1}$. In this section, we will use the notation introduced in Section 5.3 . The sets and induced maps defined there for $f$ can also be defined for $\tilde{f}$, and they will be indicated by a tilde (for instance, $\tilde{f}_{n}$, $\left.\tilde{V}_{0}^{n}, \ldots\right)$. We have

Theorem 5. There exists $\delta_{0}>0$ with the following property: Assume that $f \in$ $\mathcal{B}_{\text {nor }}(U)$ satisfies

$$
\left|\mathcal{R}^{n} f-\tilde{f}\right|_{\mathcal{B}(U)} \leq \delta \leq \delta_{0}
$$

for $n \geq 0$. Then there exists an $O(\delta)$-quasiconformal map $\phi: \mathbb{C} \rightarrow \mathbb{C}$ which is a hybrid conjugacy between $f: V_{0}^{1} \cup V_{1}^{1} \rightarrow V_{0}$ and $\tilde{f}: \tilde{V}_{0}^{1} \cup \tilde{V}_{1}^{1} \rightarrow \tilde{V}_{0}$, with $\phi\left(V_{0}\right)=\tilde{V}_{0}$.

As the first step to prove the main result of this section, we will prove

Proposition 9.1. There exists $\delta_{0}>0$ with the following property: Assume that $f \in \mathcal{B}_{\text {nor }}(U)$ satisfies

$$
\left|\mathcal{R}^{n} f-\tilde{f}\right|_{\mathcal{B}(U)} \leq \delta \leq \delta_{0}
$$

for $n \geq 0$. Then there exists a topological conjugacy $\phi: \mathbb{C} \rightarrow \mathbb{C}$ between

$$
f: V_{0}^{1} \cup V_{1}^{1} \rightarrow V_{0} \text { and } \tilde{f}: \tilde{V}_{0}^{1} \cup \tilde{V}_{1}^{1} \rightarrow \tilde{V}_{0}
$$

such that

- $\phi\left(V_{0}\right)=\tilde{V}_{0}$,

- $\phi$ is $O(\delta)$-quasiconformal outside $K(f)$.

Lemma 9.1. There exist $\delta_{0}>0, \epsilon_{0}>0$ and $C>0$ with the following property: If

$$
\left|\mathcal{R}^{n} f-\tilde{f}\right|_{\mathcal{B}(U)} \leq \delta \leq \delta_{0}
$$

for $n \geq 0$ and $\phi_{0}: A_{0} \rightarrow \tilde{A}_{0}$, where $A_{0}=\overline{V_{0}^{0}} \backslash\left(V_{0}^{1} \cup V_{1}^{1}\right)$, is an injective continuous map such that

- $\left|\phi_{0}(z)-z\right| \leq \epsilon$, for $x \in A_{0}$, for some $\epsilon$ so that $\epsilon_{0}>\epsilon>C \delta$,

- $\phi_{0} \circ f=\tilde{f} \circ \phi_{0}$ on $\partial V_{0}^{1} \cup \partial V_{1}^{1}$,

then there exist a sequence of domains $A_{n}$, the corresponding tilded domains $\tilde{A}_{n}$, and maps $\phi_{n}: A_{n} \rightarrow \tilde{A}_{n}, \psi_{n}: A_{n-1} \cup\left(f_{n-1}^{1}\right)^{-1} A_{n-1} \rightarrow \tilde{A}_{n-1} \cup\left(\tilde{f}_{n-1}^{1}\right)^{-1} \tilde{A}_{n-1}$ such that the following hold.

(1) $A_{n}:=\left(f_{n-1}^{0}\right)^{-1}\left(A_{n-1} \cup\left(f_{n-1}^{1}\right)^{-1}\left(A_{n-1}\right)\right)$.

(2) $A_{n} \subset \bar{V}_{0}^{n} \backslash\left(V_{0}^{n+1} \cup V_{1}^{n+1}\right)$ and $\partial V_{0}^{n}, \partial V_{0}^{n+1}, \partial V_{1}^{n+1}$ are connected components of $\partial A_{n}$. 


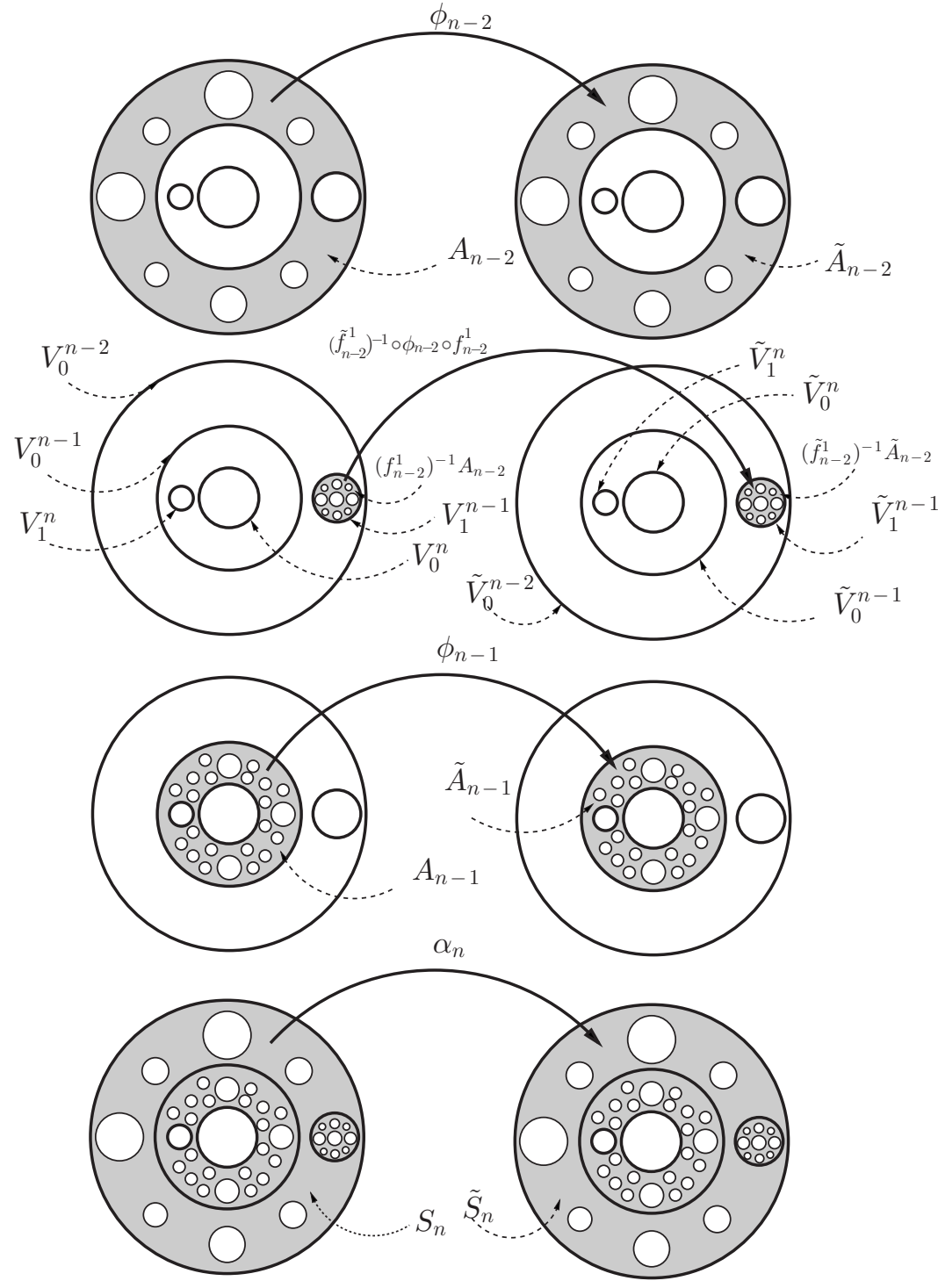

FIGURE 7.

(3) $\psi_{n}$ is equal to $\phi_{n-1}$ on $A_{n-1}$ and it coincides with $\tilde{f}_{n-1}^{-1} \circ \phi_{n-1} \circ f_{n-1}^{1}$ on $\left(f_{n-1}^{1}\right)^{-1} A_{n-1}$.

(4) $\psi_{n} \circ f_{n}^{0}=\tilde{f}_{n}^{0} \circ \phi_{n}$ on $A_{n}$ and the maps $\phi_{n-1}, \psi_{n}$ and $\phi_{n}$ coincide on $\partial V_{0}^{n}$.

(5) For $z \in \tau_{n}^{-1} A_{n}$,

$$
\operatorname{dist}\left(z, \tilde{\tau}_{n}^{-1} \phi_{n}\left(\tau_{n} \cdot z\right)\right)_{D_{0}} \leq \epsilon .
$$

Here $D_{0}$ is as in Corollary 6.1.

(6) For $z \in \tau_{n-1}^{-1}\left(A_{n-1} \cup\left(f_{n-1}^{1}\right)^{-1} A_{n-1}\right)$ we have

$$
\operatorname{dist}\left(z, \tilde{\tau}_{n-1}^{-1} \psi_{n-1}\left(\tau_{n-1} \cdot z\right)\right)_{D_{0}} \leq C \epsilon .
$$


Proof. Recall that we assume that all renormalizations of $f$ and $\tilde{f}$ are very close. Define $\psi_{1}$ equal to $\phi_{0}$ on $A_{0}$ and equal to $\left(\tilde{f}_{0}^{1}\right)^{-1} \circ \phi_{0} \circ f_{0}^{1}$ on $\left(f_{0}^{1}\right)^{-1}\left(A_{0}\right)$. Define $\phi_{1}$ as the unique map such that

- $\phi_{1}=\phi_{0}$ in $\partial V_{1}^{0}$ and

- $\psi_{1} \circ f_{1}^{0}=\tilde{f}_{1}^{0} \circ \phi_{1}$ on $A_{1}$.

$\phi_{1}$ is well defined if $\epsilon_{0}+\delta_{0}$ is small enough. Since the first renormalizations of $f$ and $\tilde{f}$ are very close, we can conclude that $\phi_{1}$ and the extension of $\phi_{0}$ are $O\left(\delta_{0}+\epsilon_{0}\right)$-close to the identity. Assume by induction that we have defined $\phi_{i}, i<n$, with the required properties. Define $\psi_{n-1}$ equal to $\phi_{n-2}$ on $A_{n-2}$ and as $\left(\tilde{f}_{n-2}^{1}\right)^{-1} \circ \phi_{n-2} \circ f_{n-2}^{1}$ on $\left(f_{n-2}^{1}\right)^{-1} A_{n-2}$. Denote $S_{n}:=A_{n-2} \cup A_{n-1} \cup\left(f_{n-2}^{1}\right)^{-1} A_{n-2}$. Let $\alpha_{n}: S_{n} \rightarrow \tilde{S}_{n}$ be the map which is equal to $\psi_{n-1}$ on $A_{n-2} \cup\left(f_{n-2}^{1}\right)^{-1} A_{n-2}$ and equal to $\phi_{n-1}$ on $A_{n-1}$ (Figure 7 describes how $\alpha_{n}$ is defined on each part of $S_{n}$ ). Then define $\phi_{n}: A_{n} \rightarrow \tilde{A}_{n}$ as the unique map (see Figure 8) such that

- $\alpha_{n} \circ f_{n-2}^{0} \circ f_{n-1}^{0}=\tilde{f}_{n-2}^{0} \circ \tilde{f}_{n-1}^{0} \circ \phi_{n}$ on $A_{n}$ and

- $\phi_{n}=\phi_{n-1}$ on $\partial V_{0}^{n}$.

Note that $\phi_{n}$ exists because $\tau_{i}^{-1} f_{i}^{0}\left(\tau_{i} z\right)$ is $\delta$-close to $\tilde{f}^{0}(z)=\tilde{\tau}_{i}^{-1} \tilde{f}_{i}^{0}\left(\tilde{\tau}_{i} z\right)$ and $\tilde{\tau}_{n-2}^{-1} \alpha_{n}\left(\tau_{n-2} z\right)$ is $O(\epsilon+\delta)$-close to the identity. Recall that

$$
g(z):=\tilde{\tau}_{i}^{-1} \tilde{f}_{i}^{0}\left(\tilde{\tau}_{i+1} z\right)
$$

is a polynomial-like map with a period two attractor, which does not depend on $i$. In particular, $\tilde{f}_{n-2}^{0} \circ \tilde{f}_{n-1}^{0}(z)=\tilde{\tau}_{n-2} g^{2}\left(\tilde{\tau}_{n}^{-1} z\right)$. Denote

$$
g_{i}(z):=\tau_{i}^{-1} f_{i}^{0}\left(\tau_{i+1} z\right)
$$

Since all renormalizations of $f$ are $\delta$-close to $\tilde{f}$, Corollary 6.1 remains valid replacing $g^{2}$ by $g_{n-2} \circ g_{n-1}=\tau_{n-2}^{-1} f_{n-2}^{0} \circ f_{n-1}^{0}\left(\tau_{n} z\right)$. We claim that

$$
\operatorname{dist}\left(z, \tilde{\tau}_{n-2}^{-1} \alpha_{n}\left(\tau_{n-2} z\right)\right)_{D_{1}} \leq \frac{\epsilon}{\lambda}+C \delta \text {, on } \tau_{n-2}^{-1} S_{n},
$$

where $\lambda>1$ does not depend on $n$. We will divide $S_{n}$ into three parts, $A_{n-2}, A_{n-1}$ and $\left(f_{n-2}^{1}\right)^{-1} A_{n-2}$, and prove the claim in each one of them.

The first part is $A_{n-2}$. Since $D_{0} \Subset D_{1}$, there exists $\lambda_{1}>1$ so that

$$
\operatorname{dist}(x, y)_{D_{1}} \leq \frac{1}{\lambda_{1}} \operatorname{dist}(x, y)_{D_{0}}, \text { if } x, y \in D_{0} .
$$

Because $\alpha_{n}$ is equal to $\phi_{n-2}$ on $A_{n-2}$, then by the inductive hypothesis, we have

$$
\operatorname{dist}\left(z, \tilde{\tau}_{n-2}^{-1} \alpha_{n}\left(\tau_{n-2} z\right)\right)_{D_{1}} \leq \epsilon / \lambda_{1}
$$

on $\tau_{n-2}^{-1} A_{n-2}$.

The second part is $A_{n-1}$. Recall that $\beta D_{0} \Subset D_{1}$, which implies that there exists $\lambda_{2}>1$ such that

$$
\operatorname{dist}(x, y)_{D_{1}} \leq \frac{1}{\lambda_{2}} \operatorname{dist}(x, y)_{\beta D_{0}}, \text { if } x, y \in \beta D_{0} .
$$

By the inductive hypothesis, we have

$$
\operatorname{dist}\left(\frac{z}{\beta_{n-1}}, \tilde{\tau}_{n-1}^{-1} \phi_{n-1}\left(\tau_{n-1} \frac{z}{\beta_{n-1}}\right)\right)_{D_{0}} \leq \epsilon
$$

on $\tau_{n-2}^{-1} A_{n-1}$. Moreover, since $\beta_{n}-\beta=O(\delta), A_{n-1} \subset \bar{V}_{0}^{n-1} \backslash\left(V_{0}^{n} \cup V_{1}^{n}\right)$ and

$$
\tau_{n-1}^{-1}\left(\bar{V}_{0}^{n-1} \backslash\left(V_{0}^{n} \cup V_{1}^{n}\right)\right) \sim K_{0} \backslash\left(\text { int } K_{1} \cup \text { int } K_{1}^{\prime}\right) \Subset D_{0},
$$




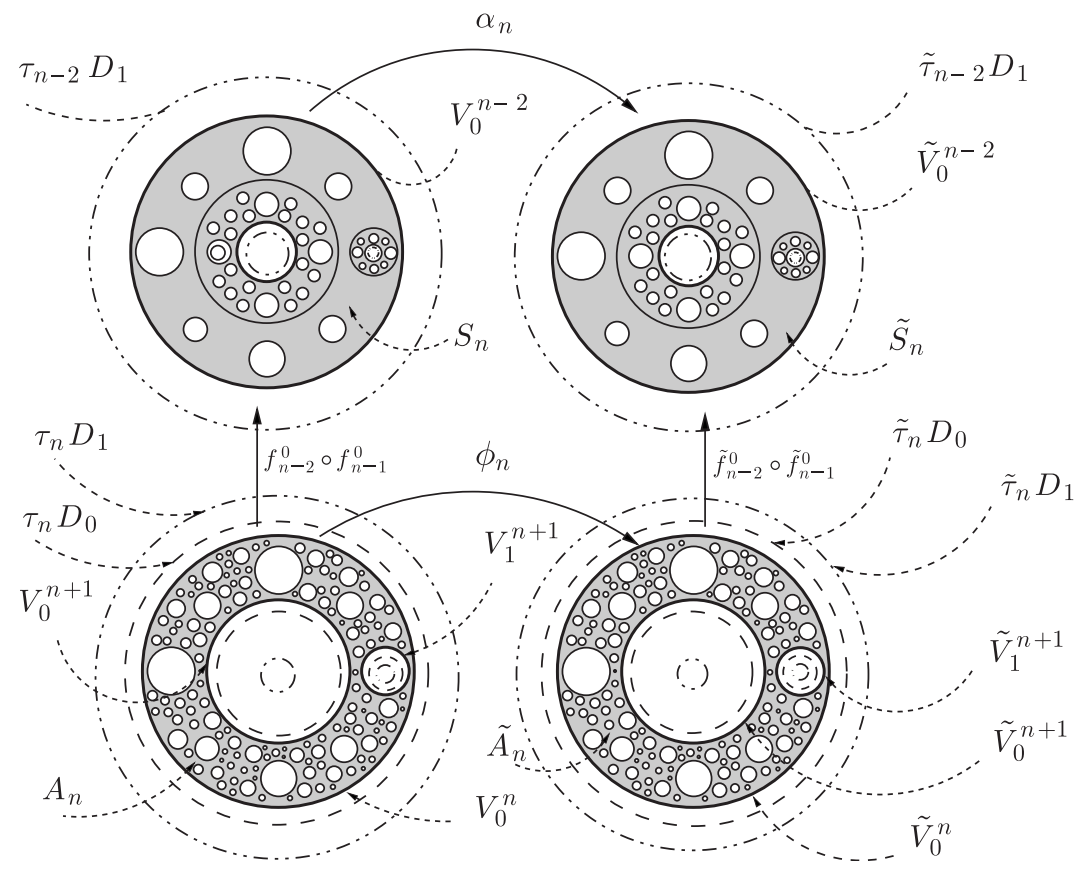

FiguRE 8.

there exists $C>0$, which does not depend on $n$, such that, for $z \in \tau_{n-2}^{-1} A_{n-1}$, we have

$$
\operatorname{dist}\left(\frac{z}{\beta}, \frac{z}{\beta_{n-1}}\right)_{D_{0}} \leq C \delta .
$$

So we have

$$
\begin{aligned}
\operatorname{dist} & \left(z, \tilde{\tau}_{n-2}^{-1} \alpha_{n}\left(\tau_{n-2} z\right)\right)_{D_{1}} \\
& =\operatorname{dist}\left(\beta \frac{z}{\beta}, \beta \tilde{\tau}_{n-1}^{-1} \phi_{n-1}\left(\tau_{n-1} \frac{z}{\beta_{n-1}}\right)\right)_{D_{1}} \\
& \leq \frac{1}{\lambda_{2}} \operatorname{dist}\left(\beta \frac{z}{\beta}, \beta \tilde{\tau}_{n-1}^{-1} \phi_{n-1}\left(\tau_{n-1} \frac{z}{\beta_{n-1}}\right)\right)_{\beta D_{0}} \\
& =\frac{1}{\lambda_{2}} \operatorname{dist}\left(\frac{z}{\beta}, \tilde{\tau}_{n-1}^{-1} \phi_{n-1}\left(\tau_{n-1} \frac{z}{\beta_{n-1}}\right)\right)_{D_{0}} \\
& \leq \frac{1}{\lambda_{2}} \operatorname{dist}\left(\frac{\beta_{n-1}}{\beta} \frac{z}{\beta_{n-1}}, \frac{z}{\beta_{n-1}}\right)_{D_{0}}+\frac{1}{\lambda_{2}} \operatorname{dist}\left(\frac{z}{\beta_{n-1}}, \tilde{\tau}_{n-1}^{-1} \phi_{n-1}\left(\tau_{n-1} \frac{z}{\beta_{n-1}}\right)\right)_{D_{0}} \\
& \leq \frac{\epsilon}{\lambda_{2}}+C \delta
\end{aligned}
$$

on $\tau_{n-2} A_{n-1}$.

The last part is $\left(f_{n-2}^{1}\right)^{-1} A_{n-2}$. Because $\tau_{n-2}^{-1} f_{n-2}^{1}\left(\tau_{n-2} z\right)$ is very close to $\tilde{f}^{1}=$ $\tilde{\tau}_{n-2}^{-1} \tilde{f}_{n-2}^{1}\left(\tilde{\tau}_{n-2} z\right)$, we have $\tau_{n-2}^{-1}\left(f_{n-2}^{1}\right)^{-1} \tau_{n-2} D_{0} \sim\left(\tilde{f}_{0}^{1}\right)^{-1} D_{0} \Subset D_{1}$, so there exists $\lambda_{3}>1$ such that

$$
\operatorname{dist}(x, y)_{D_{1}} \leq \frac{1}{\lambda_{3}} \operatorname{dist}(x, y)_{\tau_{n-2}^{-1}\left(f_{n-2}^{1}\right)^{-1} \tau_{n-2} D_{0}},
$$


for $x, y \in \tau_{n-2}^{-1}\left(f_{n-2}^{1}\right)^{-1} \tau_{n-2} D_{0}$. Here $\lambda_{3}$ does not depend on $n$. Since

$$
\tilde{f}_{n-2}^{1} \circ \alpha_{n}(z):=\phi_{n-2} \circ f_{n-2}^{1}(z)
$$

on $\left(f_{n-2}^{1}\right)^{-1} A_{n-2}$, we get

$$
\begin{aligned}
& \operatorname{dist}\left(\tau_{n-2}^{-1} f_{n-2}^{1}\left(\tau_{n-2} z\right), \tau_{n-2}^{-1} f_{n-2}^{1}\left(\tau_{n-2} \tilde{\tau}_{n-2}^{-1} \alpha_{n}\left(\tau_{n-2} z\right)\right)\right)_{D_{0}} \\
& \leq \operatorname{dist}\left(\tau_{n-2}^{-1} f_{n-2}^{1}\left(\tau_{n-2} z\right), \tilde{\tau}_{n-2}^{-1} \tilde{f}_{n-2}^{1}\left(\tilde{\tau}_{n-2} \tilde{\tau}_{n-2}^{-1} \alpha_{n}\left(\tau_{n-2} z\right)\right)\right)_{D_{0}} \\
&+\operatorname{dist}\left(\tilde{\tau}_{n-2}^{-1} \tilde{f}_{n-2}^{1}\left(\tilde{\tau}_{n-2} \tilde{\tau}_{n-2}^{-1} \alpha_{n}\left(\tau_{n-2} z\right)\right), \tau_{n-2}^{-1} f_{n-2}^{1}\left(\tau_{n-2} \tilde{\tau}_{n-2}^{-1} \alpha_{n}\left(\tau_{n-2} z\right)\right)\right)_{D_{0}} \\
& \leq \operatorname{dist}\left(\tau_{n-2}^{-1} f_{n-2}^{1}\left(\tau_{n-2} z\right), \tilde{\tau}_{n-2}^{-1} \phi_{n-2}\left(\tau_{n-2} \tau_{n-2}^{-1} f_{n-2}^{1}\left(\tau_{n-2} z\right)\right)\right)_{D_{0}} \\
&+\operatorname{dist}\left(\tilde{\tau}_{n-2}^{-1} \tilde{f}_{n-2}^{1}\left(\tilde{\tau}_{n-2} \tilde{\tau}_{n-2}^{-1} \alpha_{n}\left(\tau_{n-2} z\right)\right), \tau_{n-2}^{-1} f_{n-2}^{1}\left(\tau_{n-2} \tilde{\tau}_{n-2}^{-1} \alpha_{n}\left(\tau_{n-2} z\right)\right)\right)_{D_{0}} \\
& \leq \epsilon+C \delta
\end{aligned}
$$

on $\tau_{n-2}^{-1}\left(f_{n-2}^{1}\right)^{-1} A_{n-2}$. Because the map

$$
\tau_{n-2}^{-1} f_{n-2}^{1}\left(\tau_{n-2} \cdot\right): \tau_{n-2}^{-1}\left(f_{n-2}^{1}\right)^{-1} \tau_{n-2} D_{0} \rightarrow D_{0}
$$

is univalent, it preserves the hyperbolic metric, so we get

$$
\begin{aligned}
\operatorname{dist} & \left(z, \tilde{\tau}_{n-2}^{-1} \alpha_{n}\left(\tau_{n-2} z\right)\right)_{D_{1}} \\
& \leq \frac{1}{\lambda_{3}} \operatorname{dist}\left(z, \tilde{\tau}_{n-2}^{-1} \alpha_{n}\left(\tau_{n-2} z\right)\right)_{\tau_{n-2}^{-1}\left(f_{n-2}^{1}\right)^{-1} \tau_{n-2} D_{0}} \\
& =\frac{1}{\lambda_{3}} \operatorname{dist}\left(\tau_{n-2}^{-1} f_{n-2}^{1}\left(\tau_{n-2} z\right), \tau_{n-2}^{-1} f_{n-2}^{1}\left(\tau_{n-2} \tilde{\tau}_{n-2}^{-1} \alpha_{n}\left(\tau_{n-2} z\right)\right)\right)_{D_{0}} \\
& \leq \frac{\epsilon}{\lambda_{3}}+C \delta,
\end{aligned}
$$

on $\tau_{n-2}^{-1}\left(f_{n-2}^{1}\right)^{-1} A_{n-2}$. This finishes the proof of the claim (take $\lambda:=$ $\left.\min \left\{\lambda_{1}, \lambda_{2}, \lambda_{3}\right\}\right)$. Note that

$$
\operatorname{dist}\left(\tau_{n-2}^{-1} f_{n-2}^{0} \circ f_{n-1}^{0}\left(\tau_{n} z\right), \tilde{\tau}_{n-2}^{-1} \tilde{f}_{n-2}^{0} \circ \tilde{f}_{n-1}^{0}\left(\tilde{\tau}_{n}^{-1} z\right)\right)_{D_{1}} \leq C \delta
$$

on an open set containing $\tau_{n}^{-1} A_{n}$ and $\tilde{\tau}_{n}^{-1} \tilde{A}_{n}$. So

$$
\begin{aligned}
& \operatorname{dist}\left(\tilde{\tau}_{n-2}^{-1} \tilde{f}_{n-2}^{0} \circ \tilde{f}_{n-1}^{0}\left(\tilde{\tau}_{n} z\right), \tilde{\tau}_{n-2}^{-1} \alpha_{n}\left(f_{n-2}^{0} \circ f_{n-1}^{0}\left(\tau_{n} z\right)\right)\right)_{D_{0}} \\
& \leq \operatorname{dist}\left(\tilde{\tau}_{n-2}^{-1} \tilde{f}_{n-2}^{0} \circ \tilde{f}_{n-1}^{0}\left(\tilde{\tau}_{n} z\right), \tau_{n-2}^{-1} f_{n-2}^{0} \circ f_{n-1}^{0}\left(\tau_{n} z\right)\right)_{D_{0}} \\
& \quad+\operatorname{dist}\left(\tau_{n-2}^{-1} f_{n-2}^{0} \circ f_{n-1}^{0}\left(\tau_{n} z\right), \tilde{\tau}_{n-2}^{-1} \alpha_{n}\left(\tau_{n-2} \tau_{n-2}^{-1} f_{n-2}^{0} \circ f_{n-1}^{0}\left(\tau_{n} z\right)\right)\right)_{D_{0}} \\
& \leq \frac{\epsilon}{\lambda}+C \delta
\end{aligned}
$$

on $\tau_{n}^{-1} A_{n}$. Thus, since $g^{2}=\tilde{\tau}_{n-2}^{-1} \tilde{f}_{n-2}^{0} \circ \tilde{f}_{n-1}^{0}\left(\tilde{\tau}_{n} \cdot\right): D_{0} \rightarrow D_{1}$ is a covering map (hence it preserves the Riemanian hyperbolic metric), for each $z \in \tau_{n}^{-1} A_{n}$, there exists

$$
z_{0} \in\left(\tilde{\tau}_{n-2}^{-1} \tilde{f}_{n-2}^{0} \circ \tilde{f}_{n-1}^{0}\left(\tilde{\tau}_{n} \cdot\right)\right)^{-1}\left(\tilde{\tau}_{n-2}^{-1} \alpha_{n} \circ f_{n-2}^{0} \circ f_{n-1}^{0}\left(\tau_{n} z\right)\right)
$$

so that $\operatorname{dist}\left(z_{0}, z\right)_{D_{0}}<\epsilon / \lambda+C \delta$. Moreover, since $g^{2}: D_{0} \rightarrow D_{1}$ is a covering map, there exists $C_{1}>0$ so that

$$
\min \left\{\operatorname{dist}\left(z_{1}, z_{2}\right)_{D_{0}}: z_{1}, z_{2} \in g^{-2}(z), z_{1} \neq z_{2}, z \in \tilde{\tau}_{n-2}^{-1} \tilde{S}_{n}\right\}>C_{1},
$$

where $C_{1}$ does not depend on $n$. Now we choose $\epsilon$ so that $\epsilon / \lambda+C \delta<\epsilon<<C_{1} / 3$ (in particular $\epsilon \geq C(1-1 / \lambda) \delta$ ), and we conclude that for

$$
z_{1} \in\left(\tilde{\tau}_{n-2}^{-1} \tilde{f}_{n-2}^{0} \circ \tilde{f}_{n-1}^{0}\left(\tilde{\tau}_{n} \cdot\right)\right)^{-1}\left(\tilde{\tau}_{n-2}^{-1} \alpha_{n} \circ f_{n-2}^{0} \circ f_{n-1}^{0}\left(\tau_{n} z\right)\right),
$$


with $z_{1} \neq z_{0}$, we have $\operatorname{dist}\left(z_{1}, z\right)_{D_{0}} \geq 2 C_{1} / 3$. Since

$$
\tilde{\tau}_{n}^{-1} \phi_{n}\left(\tau_{n} z\right) \in\left(\tilde{\tau}_{n-2}^{-1} \tilde{f}_{n-2}^{0} \circ \tilde{f}_{n-1}^{0}\left(\tilde{\tau}_{n} \cdot\right)\right)^{-1}\left(\tilde{\tau}_{n-2}^{-1} \alpha_{n} \circ f_{n-2}^{0} \circ f_{n-1}^{0}\left(\tau_{n} z\right)\right)
$$

and $A_{n}$ is connected then, either

$$
\operatorname{dist}\left(z, \tilde{\tau}_{n}^{-1} \phi_{n}\left(\tau_{n} z\right)\right)_{D_{0}} \leq \epsilon \text { on } \tau_{n}^{-1} A_{n}
$$

or

$$
\operatorname{dist}\left(z, \tilde{\tau}_{n}^{-1} \phi_{n}\left(\tau_{n} z\right)\right)_{D_{0}} \geq 2 C_{1} / 3 \text { on } \tau_{n}^{-1} A_{n} .
$$

Since $\phi_{n}$ coincides with $\phi_{n-1}$ on $\partial V_{0}^{n}$, we have $\operatorname{dist}\left(z, \tilde{\tau}_{n} \phi_{n}\left(\tau_{n} z\right)\right)_{D_{0}}<O(\epsilon)<<$ $2 C_{1} / 3$, for $z \in \tau_{n}^{-1} \partial V_{n}^{0}$, by the inductive hypothesis. This completes the proof.

If $\mathcal{F}$ is a family of domains (such as $\mathcal{D}_{n}$ and $\mathcal{E}_{n}$ ), we denote $\bigcup \mathcal{F}:=\bigcup_{D \in \mathcal{F}} D$, $\mathcal{F} \cap D_{0}:=\left\{D \in \mathcal{F}: D \subset D_{0}\right\}$. The following lemma is easy:

Lemma 9.2. Let $f: \bigcup_{i} V_{i}^{1} \rightarrow V_{0}^{0}$ be a generalized polynomial-like map whose critical point does not escape and which has a sequence of critical puzzle pieces whose diameters converge to zero. Then there exists a sequence $c_{n}>0$ so that, if $D_{0} \in \mathcal{D}_{n} \cap V_{0}^{n}$, then there exists an annulus $A$ such that

- the internal boundary of $A$ is $\partial D_{0}$;

- $A \subset V_{0}^{n} \backslash \overline{U^{\mathcal{D}_{n}}}$;

- $\bmod A \geq c_{n}$.

Proof of Proposition 9.1. We again use tilde to denote the corresponding objects for $\tilde{f}$. Using holomorphic motions, it is easy to construct a $K$-quasiconformal map $\phi_{0}$ in the complex plane, with $K=O(\delta)$, such that $\phi_{0}\left(V_{0}^{0}\right)=\tilde{V}_{0}^{0}$ and $\phi_{0} \circ f=\tilde{f} \circ \phi_{0}$ on $\partial V_{0}^{1} \cup \partial V_{1}^{1}$. Apply Lemma 9.1 to $\phi_{0}$. We will prove by induction that there exist $K>0$ and a sequence of injective continuous maps $\phi_{n}: V_{0}^{0} \rightarrow \tilde{V}_{0}^{0}$ such that the following hold.

- $\phi_{n}$ coincides on $A_{n}$ with the map $\phi_{n}$ given by Lemma 9.1 .

- $\phi_{n}$ maps the set of points outside the domain of the first return map of $V_{0}^{n}$ to the corresponding set for $\tilde{f}$. Furthermore

$$
\phi_{n} \circ f=\tilde{f} \circ \phi_{n}
$$

in this set.

- $\phi_{n}$ is $K$-quasiconformal in a neighborhood of every point $x$ satisfying both of the following properties:

$-x$ does not belong to the domain of the first return map of $V_{0}^{n}$;

$-x$ is not in the Julia set.

Note that the points in the boundaries of the domains in $\mathcal{D}_{n}$ satisfy these properties.

- $\phi_{n}$ is equal to $\phi_{n-1}$ outside the domains of the first return map of $V_{0}^{n-1}$.

The pointwise limit of $\phi_{n}$ has a continuous extension to the Julia set of $f$. Moreover, it is a conjugacy between $f$ and $\tilde{f}$ which is $K$-quasiconformal outside $K(f)$.

Assume by induction that we have constructed $\phi_{n-1}$. Let $\left\{D_{j}\right\}$, with $0 \in D_{0}$, be an enumeration of the subset of domains in $\mathcal{D}_{n-1}$ which are contained in $V_{0}^{n-1}$. Enumerate the corresponding family for $\tilde{f}$ in the following way: $\tilde{D}_{j}:=\phi_{n-1} D_{j}$. Define inductively the family of domains

$$
\left\{D_{i_{1}, \ldots, i_{k}}:\left(i_{1}, \ldots, i_{k}\right) \in \mathbb{N}_{*}^{k}, \text { for some } k\right\}
$$


$\left(\mathbb{N}_{*}:=\mathbb{N}-\{0\}\right)$ in the following way:

$$
D_{i_{1}, \ldots, i_{k}}:=f_{n-1}^{-k}\left(D_{i_{k}}\right) \cap D_{i_{1}, \ldots, i_{k-1}} .
$$

Note that

$$
\bmod D_{i_{1}, \ldots, i_{k-1}} \backslash D_{i_{1}, \ldots, i_{k-1}, i_{k}} \geq c_{n-1},
$$

where $c_{n-1}$ is given in Lemma 9.2. Define $\pi_{i_{1}, \ldots, i_{k}}: D_{i_{1}, \ldots, i_{k}} \rightarrow V_{0}^{n-1}$ by $f_{n-1}^{k}$. We are going to define inductively a sequence of continuous injective maps $\psi_{n}^{k}: V_{0}^{n-1} \rightarrow$ $\tilde{V}_{0}^{n-1}$ in the following way: $\psi_{n}^{0}$ : $=\phi_{n-1}$. We define $\psi_{n}^{k}$ as equal to $\psi_{n}^{k-1}$ outside $\bigcup_{\left(i_{1}, \ldots, i_{k}\right) \in \mathbb{N}_{*}^{k}} D_{i_{1}, \ldots, i_{k}}$ and as equal to

$$
\tilde{\pi}_{i_{1}, \ldots, i_{k}}^{-1} \circ \psi_{n} \circ \pi_{i_{1}, \ldots, i_{k}}
$$

inside $D_{i_{1}, \ldots, i_{k}}$. Note that the $\psi_{n}^{k}$ are continuous and injective. We claim that the $\psi_{n}^{k}$ converge to some injective continuous map $\psi_{n}^{\infty}: V_{0}^{n-1} \rightarrow \tilde{V}_{0}^{n-1}$. To prove this, we will decompose $V_{0}^{n-1}$ into three parts, $V_{0}^{n-1}=P_{1} \cup P_{2} \cup P_{3}$.

The set $P_{1}$ consists of the points in $V_{0}^{n-1}$ whose forward orbit enters $V_{0}^{n}$ at least once. In other words, $P_{1}=\bigcup\left(\mathcal{E}_{n} \cap V_{0}^{n-1}\right)$. The sequence $\psi_{n}^{k}$ is eventually locally constant in $P_{1}$ (eventually locally constant at a point $z$ means that there exists an open set $B$, containing $z$, such that $\psi_{n}^{i}=\psi_{n}^{i+1}$ on $B$, for large $i$ ).

The set $P_{2}$ consists of the points in $V_{0}^{n-1}$ whose forward orbit returns to $V_{0}^{n-1}$ only a finite number of times and never enters $V_{0}^{n}$. Note that this set includes the boundaries of domains in $\mathcal{E}_{n}$ which are contained in $V_{0}^{n-1}$. In $P_{2}$ the sequence $\psi_{n}^{k}$ is also eventually locally constant. Moreover, if a point $z$ belongs to $P_{2} \backslash K(f)$, then we can conclude that the maps $\psi_{n}^{k}$ are $K$-quasiconformal in a neighborhood of this point, since the map $\psi_{n}^{k}$ is defined as a lift of the map $\phi_{n}$ by univalent maps, and, by the inductive hypothesis, $\phi_{n}$ is $K$-quasiconformal in a neighborhood of a large iterate of $z$.

The last set is $P_{3}$. This is the set of points in $V_{0}^{n-1}$ whose forward orbit returns to $V_{0}^{n-1}$ infinitely many times but never enters $V_{0}^{n}$. Note that $P_{3} \subset K(f)$. We will give a description of $P_{3}$ : define $p_{i_{1}, i_{2}, \ldots}$, with $\left(i_{1}, \ldots\right) \in \mathbb{N}_{*}^{\mathbb{N}}$, as the unique point in

$$
\bigcap_{k} D_{i_{1}, \ldots, i_{k}} .
$$

This set contains a unique point due to equation (51). Then $P_{3}=\left\{p_{\sigma}: \sigma \in \mathbb{N}_{*}^{\mathbb{N}}\right\}$. Note that

$$
\psi_{n}^{\ell}\left(D_{i_{1}, \ldots, i_{k}}\right) \subset \tilde{D}_{i_{1}, \ldots, i_{k}} \text {, for } \ell \text { large enough. }
$$

So, again from equation (5), we have that $\psi_{n}^{k}\left(p_{i_{1}, i_{2}, \ldots}\right) \rightarrow_{k} \tilde{p}_{i_{1}, i_{2}, \ldots}$.

Thus the pointwise limit of $\psi_{n}^{k}$, denoted by $\psi_{n}^{\infty}$, is well defined at every point in $V_{0}^{n-1}$. The continuity at the points in $P_{1} \cup P_{2}$ is obvious. The continuity at the points in $P_{3}$ is a consequence of equation (5) and equation (6). We extend the map $\psi_{n}^{\infty}$ outside $V_{0}^{n-1}$ in the following way: if $D \in \mathcal{E}_{n-1}, D \neq V_{0}^{n-1}$, define

$$
\psi_{n / D}^{\infty}:=\tilde{\pi}_{n-1 / \tilde{D}}^{-1} \circ \psi_{n}^{\infty} \circ \pi_{n-1} .
$$


Outside $\mathcal{E}_{n-1}$ define $\psi_{n}^{\infty}:=\phi_{n-1}$. Note that $\psi_{n}^{\infty}$ is continuous and injective and satisfies the following properties:

- $\psi_{n}^{\infty}$ maps the set of points outside the domain of the first entry map to $V_{0}^{n}$ to the corresponding set for $\tilde{f}$ and

$$
\psi_{n}^{\infty} \circ f=\tilde{f} \circ \psi_{n}^{\infty}
$$

in this set.

- It is $K$-quasiconformal in a neighborhood of every point $z$ which satisfies both of the properties

- $z$ does not belong to the domain of the first entry map to $V_{0}^{n}$,

- $z$ does not belong to $K(f)$.

Suppose that $f_{n-1}^{0}(0) \in D \in \mathcal{E}_{n}$. Since the maps $f_{n-1}^{0}$ and $f_{n-1}^{1}$ are very close to $\tilde{f}_{n-1}^{0}$ and $\tilde{f}_{n-1}^{1}$ and since $V_{0}^{n-1}, V_{1}^{n-1}$ and their boundaries are very close of $\tilde{V}_{0}^{n-1}$, $\tilde{V}_{1}^{n-1}$ and their boundaries, the first entry of $f_{n-1}^{0}(0)$ to $V_{0}^{n}$ occurs after applying $f_{n-1}^{1}$ once. In particular, $D$ is $\left(f_{n-1}^{0}\right)^{-1} V_{0}^{n}$, which is very close to $\left(\tilde{f}_{n-1}^{0}\right)^{-1} \tilde{V}_{0}^{n}$. Note that $\psi_{n}^{\infty}$ is equal to $\psi_{n}$ on $\partial\left(f_{n-1}^{0}\right)^{-1} V_{0}^{n}$, where $\psi_{n}$ is the map defined in Lemma 9.1 In particular

$$
\operatorname{dist}\left(z, \tilde{\tau}_{n-1}^{-1} \psi_{n}^{\infty}\left(\tau_{n-1} z\right)\right) \leq O(\delta),
$$

for $z \in \partial\left(f_{n-1}^{0}\right)^{-1} V_{0}^{n}$, which implies that $\tilde{f}_{n}^{0}(0) \in \psi_{n}^{\infty}\left(\left(f_{n-1}^{0}\right)^{-1} V_{0}^{n}\right)$ (use equation (11) in Proposition 8.11). Since $\tilde{f}_{n}^{0}(0) \in\left(\tilde{f}_{n-1}^{0}\right)^{-1} \tilde{V}_{0}^{n}$, we have $\psi_{n}^{\infty}\left(\left(f_{n-1}^{0}\right)^{-1} V_{0}^{n}\right)=$ $\left(\tilde{f}_{n-1}^{0}\right)^{-1} \tilde{V}_{0}^{n}$. Modify $\psi_{n}^{\infty}$ only inside $V_{1}^{n+1}$ to get a new map $\psi_{n}$ so that $\psi_{n}^{\infty}\left(f_{n-1}^{0}(0)\right)$ $=\tilde{f}_{n-1}^{0}(0)$, but keeping the $K$-quasiconformality of the map $\psi_{n}$ in a neighborhood of $\partial D$. Denote by $\left\{D_{j}\right\} \subset \mathcal{D}_{n}$, with $0 \in D_{0}$, the family of connected components of the preimages of the elements of $\mathcal{E}_{n} \cap V_{0}^{n-1}$ under the map $f_{n}^{0}: V_{0}^{n} \rightarrow V_{0}^{n-1}$. Define $\phi_{n}: V_{0}^{n} \rightarrow \tilde{V}_{0}^{n}$ as the unique map such that

- $\phi_{n}$ is equal to $\psi_{n}$ in $\partial V_{0}^{n}$,

- $\phi_{n} \circ f_{n}=\tilde{f}_{n} \circ \psi_{n}$ on $V_{0}^{n}-\bigcup_{i} D_{i}$.

Define $\phi_{n}:=\psi_{n}$ outside $V_{0}^{n}$. The sequence of maps $\phi_{n}$ is eventually locally constant outside $K(f)$. So we obtain a limit map $\phi: \mathbb{C} \backslash K(f) \rightarrow \mathbb{C} \backslash K(\tilde{f})$, with $\phi\left(\partial V_{0}^{0}\right)=\partial \tilde{V}_{0}^{0}$, which is a $K$-quasiconformal conjugacy. By Proposition 4.2 , $\phi$ has a continuous extension to $K(f)$.

Proof of Theorem 15. By Proposition 9.1, there exists a map $\phi: \mathbb{C} \rightarrow \mathbb{C}$ which is a conjugacy between $f$ and $\tilde{f}$, with $\phi\left(V_{0}^{0}\right)=\tilde{V}_{0}^{0}$. Moreover, $\phi$ is $O(\delta)$-quasiconformal outside $K(f)$. By Proposition 8.1, the critical pieces in the principal nest of $f$ and $\tilde{f}$ have bounded geometry and satisfy the complex bounds property (see Theorem 11), so by Theorem 1, $\phi$ is quasiconformal in the complex plane. Because $\tilde{f}$ does not support invariant line fields in $K(\tilde{f}), \bar{\partial} \phi=0$ on $K(\tilde{f})$, and hence $\phi$ is $O(\delta)$ quasiconformal in the complex plane.

\section{Part 3. Hyperbolicity of the Fibonacci cycle}

\section{INFINITESIMAL THEORY}

One of the most elegant and useful tools in Lyubich Lyu99 and Avila, Lyubich and de Melo ALdM is the theory of infinitesimal perturbations of analytic 
maps. In this section we give an introduction to this theory, including some minor simplifications and straightforward generalizations that are appropriate in our case.

Let $f$ be a generalized polynomial-like map and $\tilde{U}$ a domain compatible with $f$. The horizontal subspace $E_{f}^{h}$ comprises the vectors $v \in T \mathcal{B}_{\text {nor }}(\tilde{U})\left(E_{f}^{h}\right.$ depends on $\tilde{U}$, but we will omit this in the notation) so that there exists a quasiconformal vector field $\alpha: \mathbb{C} \rightarrow \mathbb{C}$, with $\bar{\partial} \alpha=0$ on $K(f)$, with

$$
v=\alpha \circ f-D f \cdot \alpha
$$

in a neighborhood of $K(f)$. The horizontal vectors come from quasiconformal deformations of generalized polynomial-like maps. See Lyu97 and also Sm02 for details.

The vertical subspace $E_{f}^{v}$ comprises the vectors $v \in T \mathcal{B}_{\text {nor }}(\tilde{U})$ so that there exists a holomorphic vector field $\alpha$ defined in $\overline{\mathbb{C}}-K(f)$, with $\alpha(\infty)=0$, satisfying

$$
v=\alpha \circ f-D f \cdot \alpha
$$

in some neighborhood of $K(f)$ in $\overline{\mathbb{C}}-K(f)$. The horizontal and vertical spaces were introduced by M. Lyubich.

If $|\cdot|_{\mathbb{C}}$ denotes the Riemannian spherical metric and $\beta$ is a vector field on $\bar{U} \subset \overline{\mathbb{C}}$, define $|\beta|_{\text {sph } \bar{U}}:=\sup \left\{|\beta(x)|_{\overline{\mathbb{C}}}: \quad x \in \bar{U}\right\}$.

Proposition 10.1 ([Lyu9 $)$. Let $f \in \mathcal{B}_{\text {nor }}(U)$ such that $f$ has a generalized polynomial-like extension compatible with $U$. Let $V \Subset U$ be a neighborhood of $K(f)$ so that $f^{-1}(V) \Subset V$. Then there exists $C$ such that, for any function $g$ close to $f$ so that 0 does not escape from $V$ and for $v=\alpha \circ g-D g \cdot \alpha \in E_{g}^{v}$, we have

$$
|\alpha|_{\text {sph } \overline{\mathbb{C}} \backslash V} \leq C|v|_{\mathcal{B}(V)} .
$$

Corollary 10.1. The vertical space $E_{f}^{v}$ is finite-dimensional in any Banach slice compatible with $f$. Furthermore $\operatorname{dim} E_{f}^{v}$ does not depend on the Banach slice.

Proof. It is sufficient to prove that the unit ball on $E_{f}^{v}$ is compact. Indeed, let $v_{n}=\alpha_{n} \circ f-D f \cdot \alpha_{n} \in E_{f}^{v}$, with $|v|_{\mathcal{B}(U)} \leq 1$. Let $V$ be as in Proposition 10.1 and define $V_{i}:=f^{-i} V$. Then there exists a sequence $C_{i}$ so that

$$
\left|\alpha_{n}\right|_{s p h} \overline{\mathbb{C}} \backslash V_{i} \leq C_{i} .
$$

In particular, there is a subsequence $n_{k}$ and a conformal vector field $\alpha_{\infty}$ on $\overline{\mathbb{C}} \backslash K(f)$ so that $\alpha_{i} \rightarrow \alpha_{\infty}$, where the convergence is uniform on compact sets in $\overline{\mathbb{C}} \backslash K(f)$. Furthermore, we can assume that $v_{n_{k}}$ converges uniformly to a conformal vector field $v_{\infty}$ in a neighborhood of $K(f)$ (for instance, in $V_{1}$ ). So we have

$$
v_{\infty}=\alpha_{\infty} \circ f-D f \cdot \alpha_{\infty}
$$

on $V_{1} \backslash K(f)$. Consider $\tilde{U}, U \Subset \tilde{U}$, so that $f$ has an analytical extension to $\tilde{U}$. We use equation (77) to extend $v_{i}$ and $v_{\infty}$ to $\tilde{U}$. By the maximum principle and Proposition 10.1

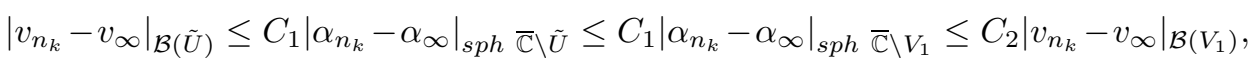

which finishes the proof: the invariance of the dimension is an immediate consequence of equation (7), since any vertical vector defined in a domain $U$ has an analytical extension to a larger domain $\tilde{U}$. 
Let $f$ be a generalized polynomial-like map and let $v$ be a domain compatible with $f$. Denote $P(f):=\overline{\left\{f^{i}(0): i>0\right\}}$. If $v \in T \mathcal{B}(U)$, we say that a quasiconformal vector field $\alpha$ is $(f, v)$-equivariant on $P(f)$ if $v(0)=\alpha(f(0))$ and

$$
\alpha\left(x_{0}\right)=\lim _{x \rightarrow x_{0}} \frac{v(x)-\alpha(f(x))}{D f(x)},
$$

for $x_{0} \in P(f)$. The concept of equivariance was introduced in Avila, Lyubich and de Melo ALdM]. The right side of the equation is well defined at the critical point (the unique problematic point) because $v(0)=\alpha(f(0))$ and $\alpha$ is quasiconformal: see [ALdM]. Indeed, we always have $\lim _{x \rightarrow 0}(v(x)-\alpha(f(x))) / D f(x)=0$. In particular, if $0 \in P(f)$ and $\alpha$ is $(f, v)$-equivariant on $P(f)$, then $\alpha(0)=0$.

The Sullivan pullback argument has an infinitesimal counterpart:

Proposition 10.2 (Infinitesimal pullback argument [ALdM]). Let $f$ be a generalized polynomial-like map compatible with the domain $U$, whose critical point does not escape. Let $V \Subset U$ be such that $f: f^{-1} V \rightarrow V$ is a representation of $f$. Then there exist $\epsilon>0$ and $C>0$ so that, if $|g-f|_{\mathcal{B}(U)} \leq \epsilon$ and $g: g^{-1} V \rightarrow V$ is a generalized polynomial-like map whose critical point does not escape, then, for any vector $v \in T \mathcal{B}_{\text {nor }}$ that admits a $(g, v)$-equivariant quasiconformal vector field in $P(f)$, there exists a quasiconformal vector field $\alpha: \overline{\mathbb{C}} \rightarrow \overline{\mathbb{C}}, \alpha(\infty)=\alpha(1)=0$, so that $v=\alpha \circ g-D g \cdot \alpha$ on $\bar{V}$ and

$$
|\bar{\partial} \alpha|_{V \backslash K(g)} \leq C|v|_{\mathcal{B}(U)} .
$$

Corollary 10.2 (Semicontinuity of the horizontal subspaces [ALdM]). Let $f, f_{n}$ be generalized polynomial-like maps which are compatible with the Banach slice $\mathcal{B}_{\text {nor }}(U)$ and so that $f_{n} \rightarrow f$ in $\mathcal{B}_{\text {nor }}(U)$. Assume that $f$ does not support invariant line fields in $K(g)$. If $v_{n} \in E_{f_{n}}^{h} \subset T \mathcal{B}(U)$ and $v_{n} \rightarrow v$ in $T \mathcal{B}_{\text {nor }}(U)$, then $v \in E_{f}^{h}$. In particular $E_{f}^{h}$ is closed.

The infinitesimal version of the Douady and Hubbard decomposition of the dynamics of a polynomial-like map in the hybrid class and its external map is the following:

Proposition 10.3 (Lyubich Decomposition Lyu99). In a Banach slice $\mathcal{B}_{\text {nor }}(U)$ compatible with $f, T \mathcal{B}_{\text {nor }}(U)=E_{f}^{h} \oplus E_{f}^{v}$.

M. Lyubich stated the above decomposition for quadratic polynomial-like maps (connected domain), but the proof can be carried out in our case as well.

The proof of Corollary 10.1 does not tell us anything about the finite dimension of $E_{f}^{v}$. The following result will be very useful to solve this problem. The proof of it is a variation of a result in Lyu99.

Proposition 10.4. Let $f$ be a generalized polynomial with non-escaping critical points. Assume there are no invariant line fields on $K(f)$ and let $U$ be a domain compatible with $f$. Then there exists $\epsilon>0$ so that, for any generalized polynomiallike map g, with non-escaping critical points and $\epsilon$-close to $f$ on $\mathcal{B}_{\text {nor }}(U)$, we have $\operatorname{dim} E_{f}^{v}=\operatorname{dim} E_{g}^{v}$.

Proof. Firstly, suppose that there exists $f_{n} \rightarrow f$ on $\mathcal{B}_{\text {nor }}(\tilde{U})$, with $\operatorname{dim} E_{f}^{v}>$ $\operatorname{dim} E_{f_{n}}^{v}$. Then, for every $n$ we can find $v_{n} \in T \mathcal{B}_{n o r}(U)$ so that $v_{n} \in E_{f}^{v} \cap E_{f_{n}}^{h}$ and $\left|v_{n}\right|_{\mathcal{B}(U)}=1$. Since $E_{f}^{v}$ is finite dimensional, we can assume that $v_{n} \rightarrow v \in E_{f}^{v}$, 
$v \neq 0$. But, by the semi-continuity of the horizontal spaces, $v \in E_{f}^{h}$, which is a contradiction. To finish the proof, it is sufficient to prove that, if there exists a sequence of maps $f_{n} \rightarrow f$ so that $\operatorname{dim} E_{f_{n}}^{v} \geq N$, then $\operatorname{dim} E_{f}^{v} \geq N$. Indeed, by a classical lemma by Riesz, there exist "almost orthonormal" families of vectors $\left\{v_{n}^{1}, \ldots, v_{n}^{N}\right\} \subset E_{f_{n}}^{v}$. In other words, $\left|v_{n}^{i}\right|_{\mathcal{B}(U)}=1$ and, if we denote

$$
\operatorname{span}\left\{u_{1}, \ldots, u_{N}\right\}:=\left\{\sum c_{i} u_{i}: c_{i} \in \mathbb{C}\right\},
$$

we have

$$
\operatorname{dist}\left(v_{n}^{i}, \operatorname{span}\left\{v_{n}^{1}, \ldots, v_{n}^{i-1}\right\}\right)_{\mathcal{B}(U)} \geq \frac{1}{2},
$$

for $1<i \leq N$. Let $\hat{U} \Subset U$. Since $\left|v_{n}^{i}\right|_{\mathcal{B}(U)}=1$, we can assume that $v_{\infty}^{i} \rightarrow v_{\infty}^{i}$ on $T \mathcal{B}_{\text {nor }}(\hat{u})$, for some vectors $v_{n}^{\infty}$. By Proposition 10.1

- $v_{\infty}^{i} \in E_{f}^{v} \backslash\{0\}$ and

- $\operatorname{dist}\left(v_{\infty}^{i}, \operatorname{span}\left\{v_{\infty}^{1}, \ldots, v_{\infty}^{i-1}\right\}\right)_{\mathcal{B}(U)} \geq C>0$, for $1<i \leq N$.

So $\operatorname{dim} E_{f}^{v} \geq N$.

Denote $\mathcal{O}(x):=\left\{f^{i}(x): i \geq 0\right\}$. The following is a slight variation of a similar result for maps with strictly preperiodic critical point in ALdM]:

Proposition 10.5. Let $f$ be a generalized polynomial-like map with a unique critical point, which is $N$-periodic. Then, if $U$ is compatible with $f$, then $v \in E_{f}^{h}$ if and only if there is a vector $\alpha: \mathcal{O}(0) \rightarrow \mathbb{C}$ so that $v=\alpha \circ f-D f \cdot \alpha$, with $\alpha(0)=0$. In particular, codim $E_{f}^{h}=1$.

Proof. It is obvious that the assumptions under $\alpha$ are necessary. We will prove that they are sufficient. Replace $\alpha$ by a quasiconformal vector field on $\overline{\mathbb{C}}$ that satisfies the same hypothesis. Then $\alpha$ is equivariant with respect to $(f, v)$ on $P(f)$. We can also assume that $\alpha$ is analytic in a neighborhood of $\mathcal{O}(0)$. It is easy to see in the proof of the infinitesimal pullback argument ALdM that there exists a quasiconformal vector field $\alpha: \mathbb{C} \rightarrow \mathbb{C}$, analytic in the interior of $K(f)$, so that $v=\alpha \circ f-D f \cdot \alpha$ on a neighborhood of $K(f)$. Since $m(J(f))=0, v \in E_{f}^{h}$. To prove that codim $E_{f}^{h}=1$, denote $v_{i}=v\left(f^{i}(0)\right)$ and $\alpha_{i}=\alpha\left(f^{i}(0)\right)$. Then

$$
v_{i}=\alpha_{i+1} \bmod n-D f\left(f^{i}(0)\right) \cdot \alpha_{i} .
$$

Since the determinant of this system of equations is 1 , for each $N$-tuple $\left(v_{0}, \ldots\right.$, $\left.v_{N-1}\right)$ there exists a unique solution $\alpha_{j}\left(v_{0}, \ldots, v_{N-1}\right)$. By the above characterization of $E_{f}^{h}$, the horizontal space is the kernel of the non-zero functional

$$
v \rightarrow \alpha_{0}\left(v(0), \ldots, v\left(f^{N-1}(0)\right)\right) .
$$

Corollary 10.3. Let $f$ be a generalized polynomial-like map with a unique critical point; assume that there are no invariant line fields on $K(f)$ and let $U$ be a domain compatible with $f$. Assume that there exists a sequence of generalized polynomiallike maps with representations in $\mathcal{B}_{\text {nor }}(U)$ so that $f_{n}$ has a superattractor and $f_{n} \rightarrow$ $f$ on $\mathcal{B}_{\text {nor }}(U)$. Then $\operatorname{dim} E_{f}^{v}=1$.

Proof. Propositions 10.4 and 10.5 
A generalized polynomial-like map $f_{1}: \bigcup_{i} U_{1} \rightarrow V_{1}$ is hybrid conjugate to another $f_{2}: \bigcup_{i} U_{2} \rightarrow V_{2}$ if there exists a quasiconformal map $h: \mathbb{C} \rightarrow \mathbb{C}$ so that

- $h\left(V_{1}\right)=V_{2}$,

- $h \circ f_{1}=f_{2} \circ h$ on $V_{1}$,

- $\bar{\partial} h$ vanishes at $K\left(f_{1}\right)$.

The hybrid class of $f_{1}$ is the set of generalized polynomial-like maps which are hybrid conjugate to $f_{1}$.

Corollary 10.4. Suppose that $f_{1}$ has a unique critical point, which does not escape, and $K\left(f_{1}\right)$ does not support invariant line fields. Assume that $f_{2}$ is a generalized polynomial-like map which is hybrid conjugate to $f_{1}$. Then the codimension of $E_{f_{2}}^{h}$ in an arbitrary Banach slice compatible with $f_{2}$ is equal to the codimension of $E_{f_{1}}^{h}$.

Proof. Indeed, let $h$ be a quasiconformal homeomorphism between two generalized polynomial-like maps $f_{a}$ and $f_{b}$ in the same hybrid class. So $h$ induces a Beltrami path $h_{\lambda} \circ f_{a} \circ h_{\lambda}^{-1}\left(h_{0}=I d\right.$ and $\left.h_{1}=h\right)$. Given two Banach slices $\mathcal{B}\left(U_{a}\right)$ and $\mathcal{B}\left(U_{b}\right)$ compatible with $f_{a}$ and $f_{b}$, we can find, using the compactness of $[0,1]$ and Proposition 10.4, a sequence of Banach slices $U_{a}=\hat{U}_{0}, \ldots, \hat{U}_{n}=U_{b}$ and closed intervals $I_{i} \subset[0,1]$, with $0 \in I_{0}$ and $1 \in I_{n}, I_{i} \cap I_{i+1} \neq \phi$, so that

- for $\lambda \in I_{i}, f_{\lambda}$ is compatible with $\mathcal{B}_{\text {nor }}\left(\hat{U}_{i-1}\right), \mathcal{B}_{\text {nor }}\left(\hat{U}_{i}\right)$ and $\mathcal{B}_{\text {nor }}\left(\hat{U}_{i+1}\right)$;

- $\operatorname{codim} E_{f_{\lambda_{0}}}^{h}=\operatorname{codim} E_{f_{\lambda_{1}}}^{h}$ on $\mathcal{B}_{\text {nor }}\left(\hat{U}_{i}\right)$, for $\lambda_{0}, \lambda_{1} \in I_{i}$.

This completes the proof.

\section{INDUCED MAPS}

In this section we study induced transformations in quite a general setting: induced transformations include the classical renormalization operator and the Fibonacci renormalization operator.

Let $f$ be a generalized polynomial-like map, let $\mathcal{B}(U)$ be a Banach slice compatible with $f$ and let $\beta_{g}$ be a non-zero analytical function defined in a neighborhood of $f$ in $\mathcal{B}(U)$ ( $\beta_{g}$ will be called the normalization function or simply 'normalization'). Consider simply connected domains $U_{1}, \ldots, U_{n}$ and $j_{1}, \ldots, j_{n} \in \mathbb{N}$ so that

- the closures of $U_{i}$ are disjoint and

- the sets $\overline{U_{i}}, \ldots, f^{j_{i}}\left(\overline{U_{i}}\right)$ are contained in $U$.

Define $\hat{U}_{i}=1 / \beta_{f} U_{i}$. Then we define a map on a neighborhood of $f$ in $\mathcal{B}(U)$ in the Banach slice $\mathcal{B}\left(\hat{U}_{1} \cup \cdots \cup \hat{U}_{n}\right)$ in the following way: for each $g$ in a small neighborhood of $f$ in $\mathcal{B}(U)$ define $\mathcal{R} g \in \mathcal{B}\left(\hat{U}_{1} \cup \cdots \cup \hat{U}_{n}\right)$ as

$$
\mathcal{R} g(x)=\frac{1}{\beta_{g}} g^{j_{i}}\left(\beta_{g} x\right), \text { for } x \in \hat{U}_{i} .
$$

The map $\mathcal{R}$ is called the (normalized) induced transformation associated with the $2 n$-tuple $\left(U_{1}, j_{1}, \ldots, U_{n}, j_{n}\right)$ and the normalization $\beta$. The set $U_{1} \cup \cdots \cup U_{n}$ is the support of the induced transformation.

Let $v \in \mathcal{B}(U)$ be a vector. Denote by $a_{n}$ the following functions:

$$
\begin{gathered}
a_{1}(x):=v(x), \\
a_{n}(x):=v\left(f^{n-1}(x)\right)+D f\left(f^{n-1}(x)\right) a_{n-1}(x) .
\end{gathered}
$$


Then (replacing $\beta_{f}$ by $\beta$ to simplify the notation)

$$
\begin{aligned}
\left(D \mathcal{R}_{f} \cdot v\right)(x) & =\frac{\partial}{\partial t}{ }_{t=0} \frac{1}{\beta_{f+t v}}(f+t v)^{n}\left(\beta_{f+t v} x\right) \\
& =\frac{-D \beta \cdot v}{\beta} \frac{1}{\beta} f^{n}(\beta x)+\frac{1}{\beta}\left(a_{n}(\beta x)+D f^{n}(\beta x)(D \beta \cdot v) x\right) .
\end{aligned}
$$

This is an ugly expression, but in the horizontal space we have a much nicer description of the action of $D \mathcal{R}$ :

Proposition 11.1. Let $\mathcal{R}$ be a normalized induced transformation with normalization $\beta_{f}$. If $v=\alpha \circ f-D f \cdot \alpha \in E_{f}^{h}$, then

$$
D \mathcal{R}_{f} \cdot v=r(\alpha) \circ \mathcal{R} f-D(\mathcal{R} f) \cdot r(\alpha),
$$

where

$$
r(\alpha):=\frac{1}{\beta_{f}} \alpha\left(\beta_{f} x\right)-\frac{1}{\beta_{f}}\left(D \beta_{f} \cdot v\right) \cdot x .
$$

Furthermore, if $\beta_{f}$ is the analytic continuation of a non-parabolic period point, then

$$
r(\alpha):=\frac{1}{\beta_{f}} \alpha\left(\beta_{f} x\right)-\frac{1}{\beta_{f}} \alpha\left(\beta_{f}\right) \cdot x .
$$

Proof. Assume $v=\alpha \circ f-D f \cdot \alpha$. Substituting this expression in the definition of $a_{n}$, we obtain by induction that, for $n>1$,

$$
a_{n}=\alpha \circ f^{n}-D f^{n} \cdot \alpha .
$$

It follows that $D \mathcal{R} \cdot v$ can be rewritten as $D \mathcal{R}_{f} \cdot v=r(\alpha) \circ \mathcal{R} f-D(\mathcal{R} f) \cdot r(\alpha)$, where

$$
r(\alpha)(x):=\frac{1}{\beta} \alpha(\beta x)-\frac{1}{\beta}(D \beta \cdot v) x .
$$

If we choose $\beta_{f}$ to be the analytic continuation of a non-parabolic periodic point of $f_{0}$ with period $i$, then we have $f^{i}\left(\beta_{f}\right)=\beta_{f}$, so

$$
D \beta_{f} \cdot v=a_{i}(\beta)+D f^{i}(\beta) D \beta_{f} \cdot v=\alpha(\beta)-D f^{i}(\beta) \alpha(\beta)+D f^{i}(\beta) D \beta_{f} \cdot v ;
$$

hence $D \beta_{f} \cdot v=\alpha(\beta)$ and

$$
r(\alpha)(x):=\frac{1}{\beta} \alpha(\beta x)-\frac{1}{\beta} \alpha(\beta) x .
$$

In particular, $v(1)=r(1)=0$.

All items in the proposition below, except the last one, are obvious generalizations of similar statements for the classic renormalization operator in de Melo and van Strien's book dMvS, Lyubich Lyu99 and Avila, Lyubich and de Melo ALdM (the second statement in Proposition [11.2 appears in Remark 7.2 in ALdM]). The third statement in Proposition 11.2 is a consequence of Proposition 11.1. The statement about the independence of induced transformations will be useful only when we are dealing with generalized polynomial-like maps with many critical points, and it will be not used here. Its proof is a straightforward generalization of the proof of the density of the image of the derivative of the classic renormalization operator [ALdM].

Proposition 11.2. Let $\mathcal{R}$ be a normalized induced transformation associated with the $2 n$-tuple $\left(U_{1}, j_{1}, \ldots, U_{n}, j_{n}\right)$ defined in a neighborhood of $f$ in a compatible $B a$ nach slice $\mathcal{B}_{\text {nor }}(U)$ in $\mathcal{B}_{\text {nor }}\left(U_{1} \cup \cdots \cup U_{n}\right)$. Then we have the following: 
(1) Compactness. The induced transformation is a compact transformation.

(2) Derivative with dense image. The derivative $D \mathcal{R}$ is a compact linear transformation with dense image.

(3) Invariance of horizontal subspaces. Assume that $f$ and $\mathcal{R} f$ have generalized polynomial-like extensions so that $\mathcal{B}_{\text {nor }}(U)$ and $\mathcal{B}_{\text {nor }}\left(U_{1} \cup \cdots \cup U_{n}\right)$ are compatible with them. Then $D \mathcal{R}_{f}\left(E_{f}^{h}\right) \subset E_{\tilde{g}}^{h}$.

(4) Non-singularity in the vertical directions. Under the conditions of the previous item, assume that $D \mathcal{R}^{-1} E_{g}^{h} \subset E_{f}^{h}$. Then

$$
\tilde{D \mathcal{R}_{f}}: T \mathcal{B}_{\text {nor }}(U) / E_{f}^{h} \rightarrow T \mathcal{B}_{\text {nor }}\left(U_{1} \cup \cdots \cup U_{n}\right) / E^{h} \tilde{g}
$$

is invertible.

Moreover we have the following:

- Independence between induced transformations. If the $\mathcal{R}_{i}, i \leq i_{0}$, are induced transformations associated with the $2 n$-tuples

$$
\left(U_{1}^{i}, j_{1}^{i}, \ldots, U_{n}^{i}, j_{n_{i}}^{i}\right),
$$

where $U_{j}^{i} \subset U$ and if the $\mathcal{R}_{i}$ have two-by-two disjoint supports, then

$$
\left\{\left(D \mathcal{R}_{1}(f) \cdot v, \ldots, D \mathcal{R}_{i}(f) \cdot v, \ldots, D \mathcal{R}_{i_{0}}(f) \cdot v\right): v \in \mathcal{B}(U)\right\}
$$

is dense in $T \mathcal{B}_{\text {nor }}\left(U_{1}^{1} \cup \cdots \cup U_{n_{1}}^{1}\right) \oplus \cdots \oplus T \mathcal{B}_{\text {nor }}\left(U_{1}^{i} \cup \cdots \cup U_{n_{i}}^{i}\right) \oplus \cdots \oplus$ $T \mathcal{B}_{\text {nor }}\left(U_{1}^{i_{0}} \cup \cdots \cup U_{n_{i_{0}}}^{i_{0}}\right)$.

\section{Contraction ON THE HYBRID Class}

Let $\tilde{f}_{i}: \tilde{V}_{0}^{1} \cup \tilde{V}_{1}^{1} \rightarrow \tilde{V}_{0}^{0}, i=1,2$, be the generalized polynomial-like extensions of $\tilde{f}_{i}$, as previously defined. Denote by $\mathcal{T}_{F i b}$, called the Fibonacci tower vSN94, the indexed family of generalized polynomial-like maps $\mathcal{R}^{i} \tilde{f}_{1}: \beta^{i} \tilde{V}_{0}^{1} \cup \beta^{i} \tilde{V}_{1}^{1} \rightarrow \beta^{i} \tilde{V}_{0}^{0}$, with $i \in \mathbb{Z}$, where either $\mathcal{R}^{i} \tilde{f}_{1}(z):=1 / \beta^{i} \tilde{f}_{1}\left(\beta^{i} \cdot z\right)$, if $i$ is even, or $\mathcal{R}^{i} \tilde{f}_{1}(z):=$ $1 / \beta^{i} \tilde{f}_{2}\left(\beta^{i} \cdot z\right)$, if $i$ is odd. This notation is consistent with the previous definitions of the Fibonacci operator $\mathcal{R}$, since this operator is injective. Let $\mu$ be a Beltrami field in $\mathbb{C}$. We say that $\mu$ is invariant by the tower $\mathcal{T}$ if

$$
\left(\mathcal{R}^{i} \tilde{f}_{1}\right)_{*} \mu=\mu
$$

for $i \in \mathbb{Z}$. We say that the Fibonacci tower $\mathcal{T}_{F i b}$ does not support invariant line fields if every Beltrami field which is invariant by $\mathcal{T}_{F i b}$ vanishes almost everywhere in $\mathbb{C}$.

Proposition 12.1 ( $[\mathrm{vSN} 94])$. The Fibonacci tower $\mathcal{T}_{\text {Fib }}$ does not support invariant line fields.

The following result can be proved using arguments as in van Strien and Nowicki vSN94]:

Proposition 12.2 (Topological convergence [vSN94]). Let $f: V_{0}^{1} \cup V_{1}^{1} \rightarrow V_{0}^{0}$ be a generalized polynomial-like map which admits a $K$-quasiconformal map $h: \mathbb{C} \rightarrow \mathbb{C}$ which is a conjugacy between $f$ and $\tilde{f}$, with $h\left(V_{0}^{0}\right)=\tilde{V}_{0}^{0}$. Then there exists $N=$ $N(K)$ so that the restriction $f_{n}: V_{0}^{n+1} \cup V_{1}^{n+1} \rightarrow V_{0}^{n}$ of the first return map to $V_{0}^{n}$ (recall the notation in Section [5.3) satisfies the following: if $\tau_{n}:=h^{-1}\left(\beta^{n}\right)$, then

$$
\frac{1}{\tau_{n}} f_{n}\left(\tau_{n} \cdot z\right)
$$


has an analytical extension to $\mathcal{B}_{\text {nor }}(U)$, provided that $n \geq N(f)$, and

$$
\left|\tau_{n}^{-1} f_{n}\left(\tau_{n} \cdot z\right)-\tilde{f}\right|_{\mathcal{B}(U)} \rightarrow 0
$$

The main result in this section is that the rate of convergence is indeed exponential. We will use the method we introduced in $\operatorname{Sm02}$. Let $\mathcal{A}(K, V, \epsilon)$ be the set of generalized polynomial-like maps $f$ in $\mathcal{B}_{\text {nor }}(U)$ so that

- $\mathcal{R}^{n} f$ is defined for $n \geq 0$ and

$$
\left|\mathcal{R}^{n} f-\tilde{f}\right|_{\mathcal{B}(U)} \leq \epsilon
$$

- there exists a $K$-quasiconformal map $\phi: \mathbb{C} \rightarrow \mathbb{C}$ so that $\phi(V) \subset \bar{U}$ and $\phi \circ \tilde{f}=f \circ \phi$ on $V$.

Note that the (closed) set $\mathcal{A}\left(K, V_{0}^{0}, \epsilon\right)$ is invariant by the action of $\mathcal{R}$, if $\epsilon$ is small enough. Moreover $\overline{\mathcal{R} \mathcal{A}\left(K, V_{0}^{0}, \epsilon\right)}$ is a compact set.

Corollary 12.1. Given $\epsilon$ small enough, there exists $K_{\epsilon}=O(\epsilon)$ so that if $f: V_{0}^{1} \cup$ $V_{1}^{1} \rightarrow V_{0}^{0}$ is a generalized polynomial-like map which admits a $\tilde{K}$-quasiconformal map $h: \mathbb{C} \rightarrow \mathbb{C}$ which is a conjugacy between $f$ and $\tilde{f}$, with $h\left(V_{0}^{0}\right)=\tilde{V}_{0}^{0}$, then, for $n \geq N(\tilde{K}), \mathcal{R}^{n} f \in \mathcal{B}_{\text {nor }}(U)$ and

$$
\mathcal{R}^{n} f \in \mathcal{A}\left(K_{\epsilon}, V_{0}^{0}, \epsilon\right) .
$$

Proof. Given $\epsilon$, by Theorem 5, there exists $K=O(\epsilon)$ so that if $f$ satisfies

$$
\left|\mathcal{R}^{n} f-\tilde{f}\right| \leq \epsilon
$$

for $n \geq 0$, then there exists a hybrid conjugacy between $f: V_{0}^{1} \cup V_{1}^{1} \rightarrow V_{0}^{0}$ and $\tilde{f}: \tilde{V}_{0}^{1} \cup \tilde{V}_{1}^{1} \rightarrow \tilde{V}_{0}^{0}$ which is $K$-quasiconformal in the complex plane. Proposition 12.2 completes the proof.

Remark 12.1. The proof of a result similar to Corollary 12.1 is easier in the classic renormalization theory for quadratic polynomials: we do not know if it is possible to prove it without Proposition 9.1. The problem is that there is not a canonical straightening for Fibonacci generalized polynomial-like maps.

Theorem 6 (Contraction in the horizontal direction). Given $\mathcal{A}\left(K, V_{0}^{0}, \epsilon\right)$, with $\epsilon$ small enough, there exist $\lambda<1$ and $N \in \mathbb{N}$ so that if $v \in E_{f}^{h}$ and $f \in \mathcal{A}(K, V, \epsilon)$, then

$$
\left|D \mathcal{R}_{f}^{N} \cdot v\right|_{\mathcal{B}(U)} \leq \lambda|v|_{\mathcal{B}(U)} .
$$

Proof. The proof is similar to the proof of Proposition 3.9 in Sm02]. Consider $f \in \mathcal{A}:=\mathcal{A}\left(K, V_{0}^{0}, \epsilon\right)$ and $v \in E_{f}^{h},|v|_{\mathcal{B}(U)} \leq 1$. Then, by the infinitesimal pullback argument, there exists a quasiconformal vector field $\alpha: \overline{\mathbb{C}} \rightarrow \overline{\mathbb{C}}$ so that $v=\alpha \circ f-$ $D f \cdot \alpha$ on $\bar{V}_{0}^{1} \cup \bar{V}_{1}^{1}$, with $|\bar{\partial} \alpha|_{\infty} \leq C$. This implies, by the definition of the Fibonacci renormalization operator, Proposition 10.2 and Proposition 11.1, that, for $n \geq 1$,

$$
D \mathcal{R}_{f}^{n} \cdot v=\alpha_{n} \circ \mathcal{R}^{n} f-D\left(\mathcal{R}^{n} f\right) \cdot \alpha_{n},
$$

on $U=U_{1} \cup U_{2}$, where

$$
\alpha_{n}(z):=\frac{1}{\beta_{\mathcal{R}^{n-1} f} \cdots \beta_{f}} \alpha\left(\beta_{\mathcal{R}^{n-1} f} \cdots \beta_{f} \cdot z\right)-\frac{1}{\beta_{\mathcal{R}^{n-1} f} \cdots \beta_{f}} \alpha\left(\beta_{\mathcal{R}^{n-1} f} \cdots \beta_{f}\right) \cdot z .
$$

Note that $\left|\bar{\partial} \alpha_{n}\right|_{\infty}=|\bar{\partial} \alpha|_{\infty} \leq C$ on $\overline{\mathbb{C}}$, for $n \geq 1$. Recall that $C$ does not depend on $f$ or $v$. Since the set of $C$-quasiconformal vectors which vanish at three points (in our case, 0,1 and $\infty$ ) in the Riemann sphere is a compact set relative to the sup 
norm induced by the spherical metric in the Riemann sphere, there exists $C>0$ so that

$$
\left|D \mathcal{R}_{f}^{n} \cdot v\right|_{\mathcal{B}(U)} \leq C
$$

for every $f \in \mathcal{A}(K, V, \epsilon), v \in E_{f}^{h},|v| \leq 1$. Since $\mathcal{R}$ is a compact operator, we have that

$$
S:=\overline{\left\{\left(\mathcal{R} f, D \mathcal{R}_{f} \cdot v\right): f \in \mathcal{A}, v \in E_{f}^{h},|v|_{\mathcal{B}(U)} \leq 1\right\}}
$$

is a compact set. Furthermore, since the horizontal subspaces are semi-continuous (Corollary 10.2), we get $S \subset\left\{(f, v): f \in \mathcal{A}, v \in E_{f}^{h}\right\}$.

We claim that, for any $v \in E_{f}^{h}, f \in \mathcal{A}$, we have

$$
\left|D \mathcal{R}_{f}^{n} \cdot v\right|_{\mathcal{B}(U)} \rightarrow 0 \text {. }
$$

It is sufficient to prove that $\alpha_{n} \rightarrow 0$. Take any convergent subsequence $\alpha_{n_{k}} \rightarrow \alpha_{\infty}$. In particular, $\bar{\partial} \alpha_{n_{k}}$ converges to $\bar{\partial} \alpha_{\infty}$ in the distributional sense. Denote $\beta_{i}:=$ $\beta_{\mathcal{R}^{i} f}$. Note that the Beltrami field $\bar{\partial} \alpha_{n}$ is invariant by the maps

$$
\mathcal{R}^{n} f, \frac{1}{\beta_{n-1}} \mathcal{R}^{n-1} f\left(\beta_{n-1} \cdot\right), \ldots, \frac{1}{\beta_{n-1} \cdots \beta_{0}} f\left(\beta_{n-1} \cdots \beta_{0} \cdot\right) .
$$

Since $\mathcal{R}^{i} f \rightarrow_{i} \tilde{f}$, this sequence of maps converges to the Fibonacci tower, which implies that $\bar{\partial} \alpha_{\infty}$ is invariant by the Fibonacci tower. Since the Fibonacci tower does not admit invariant line fields, $\alpha_{\infty}$ is a conformal vector field in the Riemann sphere. So $\alpha_{\infty}$ is zero (because $\alpha_{\infty}$ is zero at three points of the Riemann sphere: $0,1$ and $\infty)$. Fix $\delta$ small. Then, for every $(f, v) \in S$, there exists $N_{(f, v)}$ so that

$$
\left|D \mathcal{R}_{f}^{n} \cdot v\right|_{\mathcal{B}(U)} \leq \delta,
$$

for $n \geq N_{(f, v)}$. Since $\left|D \mathcal{R}_{f}^{n}\right| \leq C$, we can find a neighborhood of $B_{(f, v)}$ of $(f, v)$ in $S$ so that

$$
\left|D \mathcal{R}_{g}^{n} \cdot u\right|_{\mathcal{B}_{\text {nor }}(U)} \leq 2 C \delta,
$$

for $(g, u) \in B_{(f, v)}$ and $n \geq N_{(f, v)}$. Since $S$ is compact, this finishes the proof.

Corollary 12.2 (Contraction on the hybrid class). There exists $\lambda<1$ such that, if $f$ is a generalized polynomial-like map which is quasiconformally conjugate to one of the maps in the Fibonacci cycle, then, for $n>N=N(f)$, the nth generalized renormalization of $f$ has a representation in $\mathcal{B}_{\text {nor }}(U)$ and, furthermore,

$$
\left|\mathcal{R}^{n} f-\tilde{f}\right|_{\mathcal{B}(U)} \leq \lambda^{n} .
$$

Proof. Let $\phi: \mathbb{C} \rightarrow \mathbb{C}$ be a quasiconformal map which is a conjugacy between $f$ and $\tilde{f}$ in a neighborhood of $J(\tilde{f})$. Consider the Beltrami path induced by $\phi$ : in other words, $f_{t}:=\phi_{t} \circ \tilde{f} \circ \phi_{t}^{-1},|t| \leq 1$, where

$$
\bar{\partial} \phi_{t}=t \cdot \frac{\bar{\partial}}{\partial} \phi
$$

By the topological convergence of $\mathcal{R}^{n} f$ to $\tilde{f}$ (see vSN94]), given $\epsilon$, there exists $N=N(f, \epsilon)$ so that $\mathcal{R}^{n} f_{t} \in \mathcal{B}_{\text {nor }}(U)$ and

$$
\left|\mathcal{R}^{n} f_{t}-\tilde{f}\right|_{\mathcal{B}(U)} \leq \epsilon
$$

for $|t| \leq 1$. In particular, by Proposition 9.1 and Theorem 1, there exists $K=K(\epsilon)$ and a definitive neighborhood $V$ on $J(\tilde{f})$ so that $\mathcal{R}^{n} f_{t} \in \mathcal{A}\left(K_{\epsilon}, V_{0}^{0}, \epsilon\right)$, for $n>N_{f}$ 
and $|t| \leq 1$. Since

$$
\frac{d \mathcal{R}^{n} f_{t}}{d t} \in E_{\mathcal{R}^{n} f_{t}}^{h}
$$

we can use Theorem 6 to finish the proof.

The above argument also works for the classic renormalization operator Lyu99 (even for non-periodic combinatorics); the multimodal renormalization operator Sm01; and possibly in any other situation in complex dynamics where the McMullen arguments with towers can be applied, such as the renormalization theory for critical homeomorphisms or coverings of the circle.

\section{Hyperbolicity}

Proposition 13.1. There exists a sequence of generalized polynomial-like maps $f_{n, i}, i=1,2$, real in the real line, such that

- the map $f_{n, i}$ has a superattractor with period $S_{n}$ (the Fibonacci sequence);

- the sequence of closest returns of the critical point is exactly $f_{n, i}^{S_{i}}(0), i<n$;

- the maps $f_{n, i}$ are compatible with $U$ and $f_{n, i} \rightarrow \tilde{f}_{i}$.

In particular, codim $E_{\tilde{f}}^{h}=1$.

Proof. Consider the Fibonacci parameter $c_{\infty}$ in the family $x^{d}+c$. Select a generalized polynomial-like extension $f_{n_{0}}^{c_{\infty}}: \hat{U}_{1} \cup \hat{U}_{2} \rightarrow \hat{V}$ of a generalized renormalization of $x^{d}+c_{\infty}$, which has the Fibonacci combinatorics. There exists a unique sequence of parameters $c_{n}$ such that

- $x^{d}+c_{n}$ has a critical point with period $S_{n}$ (the Fibonacci sequence);

- the sequence of the closest return times of the critical point is $S_{i}, i<n$.

Because any accumulation point of the sequence $c_{n}$ is a parameter with Fibonacci combinatorics, we have $c_{n} \rightarrow c_{\infty}$, since, by the bounded geometry of the postcritical set, the non-existence of invariant line fields on the Julia set of Fibonacci polynomials and the Sullivan pullback argument, $c_{\infty}$ is the unique such parameter (see vSN94]). It is easy to see that $P\left(x^{d}+c_{n}\right) \rightarrow P\left(x^{d}+c_{\infty}\right)$. In particular, the $n_{0}$-th generalized renormalization of $x^{d}+c_{n}$, denoted $f_{n_{0}}^{c_{n}}$, also has a polynomial-like extension with, say, the same image $\hat{V}$ and domains $\hat{U}_{0}^{n}$ and $\hat{U}_{1}^{n}$, where these domains are very close to $\hat{U}_{0}$ and $\hat{U}_{1}$. This is sufficient to conclude that codim $E_{\tilde{f}}^{h}=1$, since $f_{n_{0}}$ is in the hybrid class of $\tilde{f}_{1}$ (or $\tilde{f}_{2}$ ). Now we could use the dynamics of the Fibonacci renormalization to conclude the proof, but we will use a more general argument: just to fix the notation, suppose that $f_{n_{0}}$ is hybrid conjugate to $\tilde{f}_{1}$. Then select a generalized polynomial-like extension $\tilde{f}_{1}: \tilde{U}_{0} \cup \tilde{U}_{1} \rightarrow \tilde{V}$ which has a representation in $U$. Then there exist $K$-quasiconformal maps, symmetric with respect to the real line,

$$
\phi_{n}: \mathbb{C}-\hat{U}_{0}^{n} \cup \hat{U}_{1}^{n} \rightarrow \mathbb{C}-\tilde{U}_{0} \cup \tilde{U}_{1}
$$

so that

- $\phi_{n}\left(\partial \hat{U}_{i}^{n}\right)=\partial \tilde{U}_{i}^{n}$

- $\phi_{n} \circ f_{n_{0}}^{c_{n}}=\tilde{f} \circ \phi_{n}$ on $\partial \hat{U}_{0} \cup \partial \hat{U}_{1}$.

Let $\mu_{n}$ be the Beltrami field which coincides with $\bar{\partial} \phi_{n}$ on $\mathbb{C}-\hat{U}_{0}^{n} \cup \hat{U}_{1}^{n}$. It is invariant under $f_{n_{0}}^{c_{n}}$ and $\mu_{n}=0$ on $K\left(f_{n_{0}}^{c_{n}}\right)$. Let $\psi_{n}$ be the $K$-quasiconformal maps, symmetric with respect to the real line, so that $\bar{\partial} \psi_{n}=\mu_{n}$. We claim that the 
generalized polynomial-like maps $\hat{f}_{n}:=\psi_{n} \circ f_{n_{0}}^{c_{n}} \circ \psi_{n}^{-1}$ converge to $\tilde{f}_{1}$. Clearly any convergent subsequence converges to a generalized polynomial-like map of the form $\hat{f}:=\psi \circ f_{n_{0}}^{c_{\infty}} \circ \psi^{-1}$. Note that the map $\phi_{n} \circ \psi_{n}^{-1}: \overline{\mathbb{C}}-\psi_{n}\left(\hat{U}_{0}^{n} \cup \hat{U}_{1}^{n}\right) \rightarrow \overline{\mathbb{C}}-\left(\tilde{U}_{0} \cap \tilde{U}_{1}\right)$ is conformal. Moreover $\phi_{n} \circ \psi_{n}^{-1} \circ \hat{f}_{n}=\tilde{f}_{1} \circ \phi_{n} \circ \psi_{n}^{-1}$ on $\psi_{n}\left(\partial \hat{U}_{0} \cup \partial \hat{U}_{1}\right)$. Taking the limit, we conclude that there exists $\phi \circ \psi^{-1}$ such that

- $\phi \circ \psi^{-1}$ is conformal outside $\psi\left(\hat{U}_{0}^{n} \cup \hat{U}_{1}^{n}\right)$,

- $\phi \circ \psi^{-1} \circ \hat{f}=\tilde{f}_{1} \circ \phi \circ \psi^{-1}$ on $\psi\left(\partial \hat{U}_{0} \cup \partial \hat{U}_{1}\right)$.

Construct a real symmetric quasiconformal map which coincides with a quasisymmetric conjugacy between $\hat{f}$ and $\tilde{f}_{1}$, on the real line, and with $\phi \circ \psi^{-1}$ on $\overline{\mathbb{C}}-$ $\psi\left(\partial \hat{U}_{0} \cup \partial \hat{U}_{1}\right)$. By the Sullivan pullback argument, we obtain a quasiconformal map $h: \overline{\mathbb{C}} \rightarrow \mathbb{C}$ which is conformal outside $K(\hat{f})$ and is a conjugacy between $\hat{f}$ and $\tilde{f}_{1}$. Since there is no invariant line field on $K(\hat{f}), h$ is an affine map, which finishes the proof.

Proposition 13.2 (No Small Orbits). There exists $\epsilon>0$ such that if $f \in \mathcal{B}_{\text {nor }}(U)$ satisfies

$$
\left|\mathcal{R}^{n} f-\tilde{f}\right|_{\mathcal{B}(U)} \leq \epsilon
$$

for $n \geq 0$, then $f$ has a generalized polynomial-like restriction which is hybrid conjugate with either $\tilde{f}_{1}$ or $\tilde{f}_{2}$ and

$$
\left|\mathcal{R}^{n} f-\tilde{f}\right|_{\mathcal{B}(U)} \leq C \lambda^{n}
$$

for some $C>0$ and $\lambda<1$.

Proof. This is an obvious consequence of Theorem 5 and Proposition 12.2

Theorem 7. The Fibonacci cycle is hyperbolic with 1-dimensional unstable manifold.

Proof. Note that, by Propositions 10.3 and 13.1, codim $E_{\tilde{f}}^{h}=1$. We already proved that $D \mathcal{R}_{\tilde{f}}^{2}$ remains invariant and is a contraction on the Banach subspace $E_{\tilde{f}}^{h}$. Note that the spectrum of $D \mathcal{R}_{\tilde{f}}^{2}$ cannot be contained inside the open disc, otherwise $\tilde{f}$ is an attracting fixed point, which implies, by Proposition 9.1 and Theorem 11, that any function in $\mathcal{B}_{\text {nor }}(U)$ very close to $\tilde{f}$ admits a quasiconformal conjugacy with $\tilde{f}$, contradicting Proposition 13.1. So, if $D \mathcal{R}_{\tilde{f}}^{2}$ is not hyperbolic, then the spectrum is contained in the closed unit disc and intersects the unit circle. By the Small Orbits Theorem Lyu99, for any small $\epsilon$, there exists $f$ so that $\left|\mathcal{R}^{i} f-\tilde{f}\right| \leq \epsilon$, for $i \geq 0$, but $\mathcal{R}^{i} f$ does not converge exponentially to $\tilde{f}$, which contradicts Proposition 13.2 . This finishes the proof.

\section{UNIVERSALITY}

In this section, we will restrict ourselves to the subspace of even maps (but we will keep the same notation for the Banach slices). This restriction is not really necessary in the universality result, but this assumption will simplify the exposition a little. Recall that the Fibonacci renormalization operator is defined in two balls $B_{1}$ and $B_{2}$ in the Banach slice $\mathcal{B}_{\text {nor }}\left(U_{0} \cup U_{1}\right)$ : the ball $B_{i}$ is an $\epsilon_{0}$-ball whose center is $\tilde{f}_{i}$. Consider the linear transformation $\phi: \mathcal{B}_{\text {nor }}\left(U_{0} \cup U_{1}\right) \rightarrow \mathcal{B}_{\text {nor }}\left(U_{0} \cup U_{1}\right)$ defined by $\phi(g)=\hat{g}$, where $\hat{g}$ coincides with $g$ on $\bar{U}_{0}$ and with $-g$ on $\bar{U}_{1}$. Then

- $\phi$ is a linear isometry of $\mathcal{B}_{\text {nor }}\left(U_{0} \cup U_{1}\right)$, 
- $\phi \circ \phi=I d$,

- $\phi \circ \mathcal{R}=\mathcal{R} \circ \phi$.

In particular $(\mathcal{R} \circ \phi)^{2}=\mathcal{R}^{2}$. Since $\phi\left(\tilde{f}_{i}\right)=\tilde{f}_{1-i}$, we have that the $\tilde{f}_{i}$ are hyperbolic fixed points of the operator $\mathcal{R} \circ \phi$. It is a question of taste to work with either $\mathcal{R}$ or $\mathcal{R} \circ \phi$ : we prefer to use the former in most of this work because its definition is more 'dynamical', which simplifies the exposition. The following lemma can be proved using the argument applied in ALdM to prove that the set of real analytic maps which are topologically conjugate with a given map $f$ is a real analytic manifold (indeed, our situation is simpler, since we will restrict ourselves to maps with a superattractor):

Proposition 14.1. There exists $C>0$ so that for each small $\delta>0$ there exists $\tilde{\delta}<\delta$ satisfying the following: Denote by $H\left(f_{n, i}\right) \subset \mathcal{B}_{n o r}(U)$ the subset of maps $f \in \mathcal{B}_{\text {nor }}(U)$ which are hybrid conjugate to $f_{n, i}$ (defined in Proposition 13.1). Then, for $n$ large enough, each connected component $\mathcal{C}$ of the intersection of $H\left(f_{n, i}\right)$ with

$$
Q_{\delta}:=\left\{\tilde{f}_{i}+v_{1}+v_{2}: v_{1} \in E_{\tilde{f}_{i}}^{h}, v_{2} \in E_{\tilde{f}_{i}}^{v},\left|v_{1}\right|_{\mathcal{B}(U)} \leq \delta \text { and }\left|v_{2}\right|_{\mathcal{B}(U)} \leq C \delta\right\}
$$

that satisfies

$$
\operatorname{dist}_{\mathcal{B}_{\text {nor }}(U)}\left(\tilde{f}_{i}, \mathcal{C}\right)<\tilde{\delta}
$$

is a connected complex analytic manifold which is the graph of a complex analytic function

$$
h_{n, i}:\left\{v_{1}: v_{1} \in E_{\tilde{f}_{i}}^{h},\left|v_{1}\right| \leq \delta\right\} \rightarrow\left\{v_{2}: v_{2} \in E_{\tilde{f}_{i}}^{v},\left|v_{2}\right| \leq C \delta\right\} .
$$

Note that $\delta, \tilde{\delta}$ do not depend on $n$.

The following corollary is a monotonicity result.

Corollary 14.1. For each small $\epsilon>0$ there exists $\tilde{\epsilon}<\epsilon$ so that $H\left(f_{n, i}\right) \cap Q_{\epsilon}$, for $n$ large enough, has a unique connected component $H_{\mathbb{R}}\left(f_{n, i}\right)$ that satisfies

$$
\operatorname{dist}_{\mathcal{B}_{\text {nor }}(U)}\left(\tilde{f}_{i}, H_{\mathbb{R}}\left(f_{n, i}\right)\right)<\tilde{\epsilon}
$$

and contains real maps.

Proof. Note that, by the Sullivan pullback argument, for each small $\epsilon$ there exists a small $\hat{\epsilon}$ so that the following holds: if two real maps belong to $H\left(f_{n, i}\right) \cap Q_{\epsilon}$, then they belong to the same connected component of $H\left(f_{n, i}\right) \cap Q_{\hat{\epsilon}}$.

Let $\tilde{\epsilon}=\tilde{\delta}$, where $\tilde{\delta}$ is as in Proposition 14.1, in such a way that it works for $\delta=\epsilon$ and $\delta=\hat{\epsilon}$. If $c_{\infty}$ is the Fibonacci parameter, then the family $x^{d}+c$ is transversal with $E_{x^{d}+c_{\infty}}^{h}$ (because this family is tangent to the vertical direction). By the contraction of the Fibonacci renormalization operator, there exists an induced transformation $\mathcal{I}$ from a neighborhood of $x^{d}+c_{\infty}$ in some Banach slice $\mathcal{B}_{\text {nor }}(V)$ ( $V$ is simply connected) to $\mathcal{B}_{n o r}(U)$ so that $\mathcal{I}\left(x^{d}+c_{\infty}\right) \in W^{s}\left(\tilde{f}_{i}\right)$ and

$$
\operatorname{dist}_{\mathcal{B}_{\text {nor }}(U)}\left(\tilde{f}_{i}, \mathcal{I}\left(x^{d}+c_{\infty}\right)\right)<\tilde{\delta}
$$

Furthermore, by the infinitesimal pullback argument and the non-existence of invariant line fields on $K\left(x^{d}+c_{\infty}\right)$, it is easy to see that $D \mathcal{I}^{-1}\left(E_{\mathcal{I}\left(x^{d}+c_{\infty}\right)}^{h}\right)=E_{x^{d}+c_{\infty}}^{h}$. So the family $\mathcal{I}\left(x^{d}+c\right),\left|c-c_{\infty}\right|<\eta$, with $\eta$ small, is transversal to $E_{\tilde{f}_{i}}^{h}$. By Proposition 14.1 if $n$ is large, the family $\mathcal{I}\left(x^{d}+c\right)$ intersects each connected component $\mathcal{C}$ of $H\left(f_{n, i}\right) \cap Q_{\hat{\epsilon}}$ satisfying

$$
\operatorname{dist}_{\mathcal{B}_{\text {nor }}(U)}\left(\tilde{f}_{i}, \mathcal{C}\right)<\tilde{\delta}
$$


at least once. This family cannot hit $H\left(f_{n, i}\right)$ in two real parameters $c_{1}, c_{2}$, since $x^{d}+c_{1}$ and $x^{d}+c_{2}$ will have the same combinatorics and, by a well-known result, for each unimodal combinatorics where the critical point is periodic, there exists only one real parameter $c$ such that $x^{d}+c$ (recall that $d$ is an even number) has this combinatorics. Let $c_{n, i}$ be such a real parameter. If a real map $f$ belongs to a connected component $\mathcal{C}$ of $H\left(f_{n, i}\right) \cap Q_{\epsilon}$ satisfying equation (9), then $f$ belongs to the connected component of $H\left(f_{n, i}\right) \cap Q_{\hat{\epsilon}}$ that contains $\mathcal{I}\left(x^{d}+c_{n, i}\right)$. By Proposition 14.1, they belong to the same connected component of $H\left(f_{n, i}\right) \cap Q_{\epsilon}$.

Proof of Theorem 3. Let $H_{\mathbb{R}}\left(f_{n, i}\right)$ be as in Corollary 14.1. Reducing $\epsilon$, if necessary, we have

and

$$
\phi\left(H_{\mathbb{R}}\left(f_{n, i}\right) \cap Q_{\epsilon}\right)=H_{\mathbb{R}}\left(f_{n, 3-i}\right) \cap Q_{\epsilon}
$$

$$
\mathcal{R}^{-1}\left(H_{\mathbb{R}}\left(f_{n-1, i}\right) \cap Q_{\epsilon}\right) \cap Q_{\epsilon} \subset H_{\mathbb{R}}\left(f_{n, 3-i}\right),
$$

so

$$
(\mathcal{R} \circ \phi)^{-1}\left(H_{\mathbb{R}}\left(f_{n-1, i}\right) \cap Q_{\epsilon}\right) \cap Q_{\epsilon} \subset H_{\mathbb{R}}\left(f_{n, i}\right) .
$$

Let $f_{\lambda} \in \mathcal{B}_{\text {nor }}\left(U_{0} \cup U_{1}\right), \lambda \in \mathbb{C}$, be a complex analytic family so that $f_{\lambda}(z)$ is real if $\lambda$ and $z$ are real. Assume that $f_{0}$ has the Fibonacci combinatorics. By the topological convergence of the Fibonacci renormalization operator, we can define an induced transformation $\mathcal{I}$ from a neighborhood of $f_{0}$ to a neighborhood of a point in the local stable manifold of $\tilde{f}_{1}$.

Since the image of the derivative of any induced transformation is dense, we can find a vector $v \in T \mathcal{B}_{\text {nor }}\left(U_{0} \cup U_{1}\right)$ so that the family $\mathcal{I}\left(f_{\lambda}+\lambda \cdot v\right)$ is transverse to $W_{l o c}^{s}\left(\tilde{f}_{1}\right)$.

The tangent space of $H_{\mathbb{R}}\left(f_{n, i}\right)$ at a point $g$ is exactly $E_{g}^{h}$. So $H_{\mathbb{R}}\left(f_{n, i}\right)$ is transversal to the unstable direction of $\tilde{f}_{i}$. Now consider the operator $\mathcal{R} \circ \phi$ and its fixed points $\tilde{f}_{i}$ and apply a well-known fact about (complex) hyperbolic fixed points with (complex) 1-dimensional complex unstable manifold, which implies that, if $f_{\lambda}$ is a curve in $\mathcal{B}_{\text {nor }}(U)$ that intersect $W_{l o c}^{s}\left(\tilde{f}_{i}\right)$ in a transversal way at $\lambda=0$, then there exists, for $n$ large enough, a unique sequence $\lambda_{n}$ (the uniqueness follows that of Corollary 14.1) such that $f_{\lambda_{n}} \in H_{\mathbb{R}}\left(f_{n, i}\right)$ and

$$
\frac{\lambda_{n}-\lambda_{n+1}}{\lambda_{n+1}-\lambda_{n+2}}
$$

converges to the unique eigenvalue of $D\left(\mathcal{R}_{\tilde{f}} \circ \phi\right)$ larger than one. Because $(\mathcal{R} \circ \phi)^{2}=$ $\mathcal{R}^{2}$, the absolute value of this eigenvalue is exactly the square root of the modulus of the unique eigenvalue larger than one of the operators $D \mathcal{R}_{\tilde{f}_{1}}^{2}$ and $D \mathcal{R}_{\tilde{f}_{2}}^{2}$ (these two linear operators have the same spectrum, as $\mathcal{R}^{2} \circ \phi=\phi \circ \mathcal{R}^{2}$ ).

For the family of generalized polynomials induced by the family $x^{d}+c$, a perturbation is not necessary: consider the induced transformation defined in the proof of Corollary 14.1. We concluded there that the family $\mathcal{I}\left(x^{d}+c\right)$ is transversal to $W_{l o c}^{s}\left(\tilde{f}_{1}\right)$. This finishes the proof.

\section{Further DEVElopments AND OPEN QUeSTIONS}

Quite a lot has happened concerning the rigidity of polynomials and puzzle geometry control since this work came out as a preprint in March 2002, in such a way that it was necessary to modify this section. With the exception of this section, some misprints, reduction of some sections (as suggested by the referee), modifications in 
Proposition 14.1, Corollary 14.1 and Theorem 3, and the modification mentioned below, the mathematical content of this article matches exactly with that preprint.

The new development is the work entitled "Rigidity for real polynomials", by O. Kozlovski, W. Shen, and S. van Strien. They claim quasiconformal rigidity for all real polynomials with only real critical points, which implies the density of hyperbolic polynomials in this class. The methods introduced in this work, in particular the use of puzzle geometry control to obtain rigidity, have a major role in their proof. For instance, they prove that all non-renormalizable real unimodal polynomials (with "non-trivial" combinatorics) have sequences of critical puzzle pieces with bounded geometry. Actually, they show that the puzzle geometry control is a property shared by essentially all real polynomials with real critical points (including, in some sense, infinitely renormalizable polynomials). In view of this, it was necessary to remove the following line from the introduction: "On the other hand, the linear decay of modulus holds for all real non-renormalizable quadratic polynomials (Lyubich Lyu94), what is no longer true to the puzzle geometry control property."

Actually, the puzzle geometry control does not hold (in general) in the critical pieces in the principal nest, but Koslowski, Shen and van Strien show that it is always possible to find a sequence of critical pieces that does satisfy this condition.

However, the following conjecture and analogous statements for polynomials with many critical points remain open:

Conjecture 1. The set of parameters $c \in \mathbb{R}$ so that $x^{d}+c$ has a wild attractor has zero Lebesgue measure.

We hope to give a first approximation to Conjecture 1, using generalized renormalization to prove that sets of parameters corresponding to large sets of combinatorics which admit wild attractors have zero Lebesgue measure in the parameter space. The idea is to prove the existence of a hyperbolic set for the generalized renormalization operator associated with these combinatorics, so that this set contains all limit behaviors of the action of the generalized renormalization operator on the maps which are infinitely renormalizable with respect to it. In view of the work of Lyubich in the quadratic family Lyu99, the existence of hyperbolic sets for generalized renormalization operators implies that the set of parameters in the real family $x^{d}+c$ whose corresponding map is infinitely renormalizable with respect to these generalized renormalization operators has zero Lebesgue measure in the parameter space (in the families $x^{d}+c$ ). The methods applied in this work to the Fibonacci renormalization operator can be generalized to a lot of other combinatorics, including non-periodic ones. Note that, to prove the hyperbolicity, one needs more than the puzzle geometry control: after normalizing the domains of the generalized renormalizations in an appropriate way, we must obtain polynomial-like maps which are hyperbolic, in such a way that we can control the critical puzzle geometry of maps whose renormalizations are very close to the original map. We also expect that this kind of argument can be generalized to many other combinatorics, including the non-periodic case.

\section{ACKNOWLEDGMENTS}

I would like to express my thanks for the hospitality of IMS-SUNY-Stony Brook, ICTP-Trieste, the Mathematics Institute of Warwick University, and IMPA, where 
parts of this work were done. I would like to thank especially M. Lyubich for my exciting period at Stony Brook and S. van Strien for my wonderful stay at Warwick. Thanks to A. Avila for discussions about Avila, Lyubich and de Melo's work and the correction of a mistake in the proof of Corollary 10.1. I am also grateful to Juan Rivera-Letelier, whose comments were useful for improving the presentation of this work, for the fruitful suggestion of an inductive argument with the hyperbolic metric in the proof of Lemma 9.1, and for his great support in the fall of 2001.

\section{REFERENCES}

[AB] L. Ahlfors and L. Bers. Riemann's mapping theorem for variable metrics. Ann. of Math., 72:385-404, 1960. MR0115006 (22:5813)

[ALdM] A. Avila, M. Lyubich and W. de Melo. Regular or stochastic dynamics in real analytic families of unimodal maps. Invent. Math., 154:451-550, 2003. MR.2018784 (2006i:37083)

[B] B. Bojarski. Remarks on Sobolev imbedding inequalities. In Proc. of the Conference on Complex Analysis, Joensuu, 1987, Lecture Notes in Math., 1351, Springer-Verlag, 1988. MR0982072 (90b:46068)

[Br] B. Branner. Cubic polynomials: turning around the connectedness locus. In Topological methods in modern mathematics (Stony Brook, NY, 1991), Publish or Perish, Houston, 1993. MR1215972 (94c:58168)

[BH98] B. Branner and J. Hubbard. The iteration of cubic polynomials. I. The global topology of parameter space. Acta Math., 160:143-206, 1998. MR0945011 (90d:30073)

[Bru] H. Bruin. Topological conditions for the existence of absorbing Cantor sets. Trans. Amer. Math. Soc., 350:2229-2263, 1998. MR1458316 (99f:58064)

[BKNS] H. Bruin, G. Keller, T. Nowicki and S. van Strien. Wild Cantor attractors exist. Ann. of Math. (2), 143:97-130, 1996. MR1370759 (96m:58145)

[Bu] X. Buff. Fibonacci fixed point of renormalization. Ergodic Theory and Dynam. Systems, 20:1287-1317, 2000. MR:1786715 (2001m:37086)

[GS96] J. Graczyk and G. Swiatek. Induced expansion for quadratic polynomials. Ann. Sci. École Norm. Sup. (4), 29:399-482, 1996. MR1386222 (98d:58152)

[GS97] J. Graczyk and G. Swiatek. Generic hyperbolicity in the logistic family. Ann. of Math. (2), 146:1-52, 1997. MR:1469316 (99b:58079)

[HK95] J. Heinonen and P. Koskela. Definitions of quasiconformality. Invent. Math., 120:61-79, 1995. MR.1323982 (96e:30051)

[KK00] S. Kallunki and P. Koskela. Exceptional sets for the definition of quasiconformality. American Journal of Mathematics, 122:735-743, 2000. MR.1771571(2001h:37095)

[KN] G. Keller and T. Nowicki. Fibonacci maps re(al)visited. Ergodic Theory and Dynam. Systems, 15:99-120, 1995. MR1314971 (95k:58098)

[LV] O. Lehto and K. Virtanen. Quasiconformal mappings in the plane. Springer-Verlag, New York-Heidelberg, 1973. MR0344463 (49:9202)

[LvS] G. Levin and S. van Strien. Local connectivity of the Julia set of real polynomials. Ann. of Math., 147:471-541, 1998. MR.1637647 (99e:58143)

[Lyu93] M. Lyubich. Teichmuller space of Fibonacci maps. Preprint, ims93-12, IMS-SUNY at Stony Brook, 1993.

[Lyu97] M. Lyubich. Dynamics of quadratic polynomials. I, II. Acta Math, 178:185-297, 1997. MR.1459261 (98e:58145)

[Lyu94] M. Lyubich. Combinatorics, geometry and attractors of quasi-quadratic maps. Ann. of Math. (2), 140:347-404, 1994. MR1298717 (95j:58108)

[Lyu99] M. Lyubich. Feigenbaum-Collet-Tresser universality and Milnor's hairness conjecture. Ann. of Math., 149:319-420, 1999. MR1689333 (2000d:37051)

[LM] M. Lyubich and J. Milnor. The Fibonacci unimodal map. J. Am. Math. Soc., 6:425-457, 1993. MR 1182670 (93h:58080)

[dMvS] W. de Melo and S. van Strien. One-dimensional dynamics. Springer-Verlag, Berlin, 1993. MR.1239171 (95a:58035)

$[\mathrm{McM}]$ C. McMullen. Renormalization and 3-manifolds which fiber over the circle. Annals of Mathematics Studies, 142, Princeton University Press, Princenton, 1996. MR1401347 (97f:57022) 
[P] R. A. Perez. On the combinatorics of the principal nest. Ph. D. Thesis, IMS-SUNY at Stony Brook, 2002.

[PR99] F. Przytycki and S. Rohde. Rigidity of holomorphic Collet-Eckmann repellers. Ark. Mat., 37:357-371, 1999. MR1714763 (2000i:37064)

[Sh] W. Shen. On the metric property of multimodal maps and $C^{2}$ density of Axiom A. Invent. Math., 156, no. 2, 301-403, 2004. MR2052610(2005d:37078)

[Sm01] D. Smania. Phase space universality for multimodal maps. Bull. Braz. Math. Soc, New Series 36(2), 225-274, 2005. MR2152018(2006f:37057)

[Sm02] D. Smania. On the hyperbolicity of the period-doubling fixed point. Trans. Amer. Math. Soc., 358, no. 4, 1827-1846, 2006. MR2186998

[vSN94] S. van Strien and T. Nowicki. Polynomial maps with a Julia set of positive Lebesgue measure: Fibonacci maps. Unpublished, 1994.

[Sul92] D. Sullivan. Bounds, quadratic differentials, and renormalization conjectures. In $A M S$ Centennial Publications: Mathematics into the Twenty-first Century, volume 2. AMS, 1992. MR $1184622(93 \mathrm{k}: 58194)$

[SV] G. Swiatek and E. Vargas. Decay of geometry in the cubic family. Ergodic Theory Dynan. Systems, 18:1311-1329, 1998. MR1653256(99h:58161)

Institute for Mathematical Sciences, State University of New York at Stony Brook, STONy Brook, New York 11794-3660

Current address: Departamento de Matemática, ICMC-USP-Campus de São Carlos, Caixa Postal 668, São Carlos-SP, CEP 13560-970, Brazil

E-mail address: smania@icmc.usp.br

$U R L$ : www.icmc.usp.br/ smania 